\title{
March 2000 Working Group Meeting on Heavy Vehicle Aerodynamic Drag: Presentations and Summary of Comments and Conclusions
}

R. McCallen, D. Flowers, T. Dunn, J. Owens, F. Browand, M. Hammache, A. Loeonard, M. Brady, K. Salari, W. Rutledge, R. Scheckler, J. Ross, B. Storms, J.T. Heineck, T. Arledge

\section{May 15, 2000}

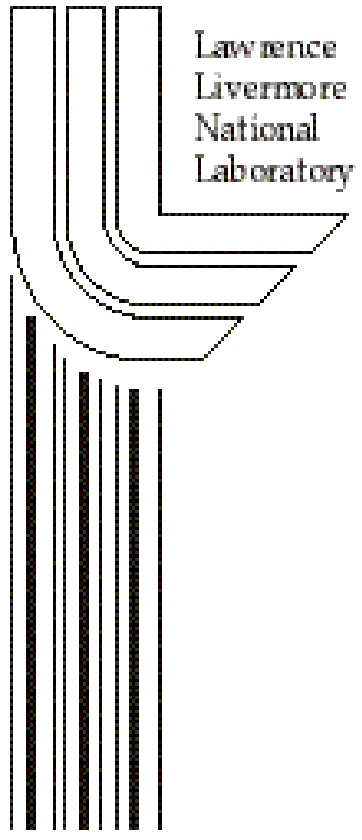




\section{DISCLAIMER}

This document was prepared as an account of work sponsored by an agency of the United States Government. Neither the United States Government nor the University of California nor any of their employees, makes any warranty, express or implied, or assumes any legal liability or responsibility for the accuracy, completeness, or usefulness of any information, apparatus, product, or process disclosed, or represents that its use would not infringe privately owned rights. Reference herein to any specific commercial product, process, or service by trade name, trademark, manufacturer, or otherwise, does not necessarily constitute or imply its endorsement, recommendation, or favoring by the United States Government or the University of California. The views and opinions of authors expressed herein do not necessarily state or reflect those of the United States Government or the University of California, and shall not be used for advertising or product endorsement purposes.

Work performed under the auspices of the U. S. Department of Energy by the University of California Lawrence Livermore National Laboratory under Contract W-7405-Eng-48.

This report has been reproduced

directly from the best available copy.

Available to DOE and DOE contractors from the

Office of Scientific and Technical Information

P.O. Box 62, Oak Ridge, TN 37831

Prices available from (423) 576-8401

http:/ /apollo.osti.gov/bridge/

Available to the public from the National Technical Information Service

U.S. Department of Commerce 5285 Port Royal Rd., Springfield, VA 22161

http://www.ntis.gov/

OR

Lawrence Livermore National Laboratory

Technical Information Department's Digital Library

http://www.llnl.gov/tid/Library.html 


\title{
March 2000 \\ Working Group Meeting on \\ Heavy Vehicle Aerodynamic Drag:
}

\section{Presentations and Summary of Comments and Conclusions}

\author{
Jointly written by \\ Lawrence Livermore National Laboratory \\ Sandia National Laboratories \\ University of Southern California \\ California Institute of Technology \\ NASA Ames Research Center
}

\section{Introduction}

A Working Group Meeting on Heavy Vehicle Aerodynamic Drag was held at Lawrence Livermore National Laboratory on March 16, 2000. The purpose of the meeting was to present technical details on the experimental and computational plans and approaches and provide an update on progress in the analysis of experimental results, model developments, simulations, and an investigation of an aerodynamic device. The focus of the meeting was a review of University of Southern California's (USC) experimental plans and results, NASA Ames experimental plans, the computational results from Lawrence Livermore National Laboratory (LLNL) and Sandia National Laboratories (SNL) for the integrated tractor-trailer benchmark geometry called the Ground Transportation System (GTS) Model, and turbulence model development and benchmark simulation for a rounded cube from California Institute of Technology (Caltech). Much of the meeting discussion involved deficiencies in commercial software, needed modeling improvements, and the importance of detailed data for code validation. The present and projected budget and funding situation was also discussed.

Presentations were given by representatives from the Department of Energy (DOE) Office of Transportation Technology Office of Heavy Vehicle Technology (OHVT), LLNL, SNL, NASA Ames, USC, and Caltech. Representatives from Argonne National Laboratory also participated via telephone. This report contains the technical presentations (viewgraphs) 
delivered at the Meeting, briefly summarizes the comments and conclusions, and outlines the future action items.

\section{Summary of Major Issues}

There were 3 major issues raised at the meeting.

1. Our funding is inadequate to satisfy industries request for high Reynolds number experimentation and computation. Plans are to respond to the DOD and DOE requests for proposals, which require a 50-50 cost share with industry, to acquire funding for high Reynolds number experiments at NASA Ames.

2. The deficiencies in commercial software, the need for model improvements and validation, and the unavailability of a detailed database for advanced model validation needs to be recognized.

3. The need for industrial collaboration appears to be a requirement for acquiring funding.

\section{Overview of the Project, Current Funding, and Future Workshop}

Jules Routbort of DOE OHVT and Argonne National Laboratory provided an overview of the OHVT budget from fiscal year (FY) 1997 through FY00 for heavy vehicle systems aerodynamic drag reduction. For projects involving heavy vehicle systems parasitic energy losses, OHVT has requested a total of \$4.5 million for FY01 with an additional \$1.5 million for the solicitation of industry proposals on parasitic energy losses. The Aero Team's estimated costs for FY 2001 is over \$1.5 million, because of the high cost for needed NASA experiments. This would require almost all of the OHVT's total budget for parasitic energy losses, which is not a reasonable expectation. Rather than an increase in funding, Jules presented a possible scenario for funding reduction for this project.

Jules emphasized that stable funding for our computational and experimental effort will require industrial support. Future funding through formal requests for proposals (under competitive bid) will likely require a 50-50 cost share from industry. Jules stated that commercial software companies have indicated that they can do what the Aero Team is pursuing. This comment set the tone for much debate, with the Aero Team providing evidence of needed model development, advanced computational resources, and experimental data for code validation. Without benchmarking and validation in a careful systematic approach, the correctness or accuracy of computations are unknown.

An overview of the project was presented by Rose McCallen of LLNL. The viewgraphs are enclosed. Budget issues were presented as well as the project calendar of events and plans for submitting proposals for needed funding. It was emphasized that several of the team participants (USC, Caltech, and NASA) had still not received or had just recently received the expected FY00 funds. Specifically, NASA had not received any funds and thus all testing was on hold and scheduling of tests for FY00 was in jeopardy.

It was emphasized that the program deliverables are being met only because of the team's 
success in leveraging funds from internal research support (e.g., LDRD and Tech Base at the National Labs) and the support of other agencies (e.g., DOD, Caltrans, NSF, ASCI) for related work.

\section{NASA's Plans for 7-ft x 10-ft Wind Tunnel Experiments in FY00 and Future Plans for 12-ft Wind Tunnel Experiments}

Jim Ross of NASA Ames presented options for the 7-ft x 10-ft wind tunnel tests which include experiments with a modified GTS model or use of a 1/8th scale model from International Transportation Corporation. NASA's plans also include provisions for USC to test their GTS model in the 7-ft x 10-ft wind tunnel for evaluation at lower blockage and higher Reynolds number flow. The purpose of all these experiments are for validation of the computational fluid dynamics (CFD) models and for further insight into truck flow phenomena.

Jim also presented details of the high Reynolds number experiments proposed for the 12-ft pressure wind tunnel. These experiments will provide answers to scaling issues as well as a database for code validation. Details are provided in the attached viewgraphs. The truck industry is very interested in these tests because they recognize that there are discrepancies between the Re effects experienced with a full-size truck and that predicted by experiment on scaled down models in wind tunnels.

The expected costs for the 12-ft wind tunnel experiments are at a 1/3rd reduced cost for a government funded effort. NASA is not charging for salaries. An additional $\$ 240 \mathrm{~K}$ could have been saved if the experiments had been performed in FY00. The sooner these experiments can be scheduled, the less the cost. Unfortunately, DOE funds are not adequate to support these needed experiments.

Experiments with a production model in the 12 -ft wind tunnel could be linked to field tests on the same geometry. This will allow for the added benefit of relating wind tunnel drag to fuel economy. However, higher Reynolds number experiments with the GTS geometry provide for an expansion of the existing database for code validation with a simplified geometry. The NASA cost estimates include the possibility of performing the experiments with both a production model and the GTS model.

Jim also mentioned an interest in using the NASA computational tools to simulate the proposed experiments. Use of the NASA code OVERFLOW was proposed. The computational tool uses a RANS approach with structured-overset grids.

\section{USC's Wind Tunnel Tests and a Look at an Aero Device}

Fred Browand of USC provided a status report of recent experiments and analysis of results. The drag results obtained in the USC wind tunnel were compared to the NASA experiments at low Reynolds number, providing explanation for any discrepancies. It was noted that the base pressure is not changing much in the low Reynolds number regime so that the drag is dominated by the front curvature. At high Reynolds number, the GTS 
geometry drag is all base drag so that there is significant leverage for improvement with wake conditioning.

Fred also present some preliminary results using an oscillation device to control the trailer wake flow. The device alters the turbulent structure of the wake resulting in a drag reduction. Proof-of-principal experiments are needed to determine the benefits of the oscillation device for drag mitigation.

\section{Computational Model Development and Simulations}

\section{RANS Computations at SNL}

An overview of the Reynolds-averaged Navier Stokes (RANS) computation being performed by SNL was presented by Kambiz Salari. Current efforts involve the modeling of the NASA experiments in the $7-\mathrm{ft} \times 10-\mathrm{ft}$ wind tunnel.

To determine the appropriate inflow conditions for the computations, the empty tunnel was fully modeled and computed velocities were compared to those measured in the empty $7-\mathrm{ft} \times 10-\mathrm{ft}$ wind tunnel. The computed and experimental velocity profiles at the test section entrance are in agreement and an adequate entrance and exit section for the computations was identified. Questions were raised as to how the pressure coefficient should be computed to be consistent with experiments.

The RANS one-equation Spalart-Allmaras turbulence model captures accurately the measured surface pressure coefficient at 0-degrees yaw for the GTS geometry. The calculations and the experiments indicate attached flow at the sides of the model for 0-degrees yaw. Some discrepancies between the computed and experimental pressure coefficient were present on the rear of the truck for 0-degrees yaw. At 10-degrees yaw, particle trace plots of the computations indicate the flow roll-up over the top of the vehicle as observed with pressure-sensitive paint (PSP) and oil-film interferometry (OIF) measurements in the wind tunnel. Details are provided in the enclosed viewgraph presentation.

\section{Large-Eddy Simulations using the Finite Element Method at LLNL}

The large-eddy simulation (LES) approach being used by LLNL was presented by Tim Dunn for both their compressible and incompressible flow models. The approach and development challenges were presented along with a progress update. Implementation of the incompressible model is complete and some validation remains. See attached viewgraphs for details on the models.

Dan Flowers of LLNL presented preliminary results using the compressible model for the GTS geometry for 0.3 and 2.4 million elements at 0-degrees yaw. A method for ease in grid refinement by using a separate near-body grid that boxes the vehicle was also presented. The computations with the course grid indicate higher drag results than measured, however, grid refinements show a trend towards the experimental results. The computed dominate pressure frequency with the 0.3 million element grid compares to that measured 
with the unsteady pressure probe mounted on the rear of the GTS. Further details are provided in the enclosed viewgraph presentation.

\section{Simulations using Vortex Methods: A Gridless Technique}

The Caltech group continues to improve their fast, parallelized, adaptive vortex method. Current activities at Caltech include: incorporating bodies with arbitrary complexity, obtaining higher Reynolds numbers computations, and developing and analyzing subgrid models for large-eddy simulation. Mark Brady of Caltech provided an overview of the vortex method approach and described a new technique for incorporating arbitrary sized body-surface triangles which has been recently implemented. This technique maintains computation accuracy while improving performance. Also a geometry definition similar to the GTS model (cab and trailer) has been defined and tested. Simulation Reynolds numbers are still quite low but plans are to move into the higher Reynolds number regime with the addition of subgrid scale models. Tony Leonard of Caltech presented his work in developing subgrid scale models for near-wall turbulence for use with large-eddy simulation. Several approaches for subgrid modeling for turbulent boundary layers have been identified for further developement.Further details and results of computations with the vortex method code and on the turbulence modeling approach are in the attached viewgraphs.

\section{Future Meetings}

The next Working Group Meeting will likely be in July or August 2000. The location of the next meeting has not yet been established.

\section{Action Items}

The follow-on action items with the individuals responsible for the tasks are as follows:

Response to DOD and DOE request for proposals (R. McCallen)

Proposal submission to BES and NSF (F. Browand and A. Leonard)

Paper and presentation at SAE Government Industry Meeting in Washington, DC in June 2000 (R. McCallen)

Meeting report with viewgraphs (R. McCallen)

Quarterly report due April 15, 2000 (R. McCallen)

Establish location and schedule next working group meeting (R. McCallen) 


\section{Truck Aero Team Meeting}

\section{LLNL, Livermore, CA}

March 16, 2000

\section{$\underline{\text { Attendee List }}$}

\begin{tabular}{|c|c|c|}
\hline Attendee & Organization & e-mail address and phone \\
\hline Tom Arledge & NASA & tarledge@mail.arc.nasa.gov, 650-604-1604 \\
\hline Mark Brady & Caltech & mbrady@caltech.edu, 626-395-3285 \\
\hline Fred Browand & USC & browand@ @spock.usc.edu, 213-740-5359 \\
\hline Tim Dunn & LLNL & tdunn@Ilnl.gov, 925-422-8258 \\
\hline Dan Flowers & LLNL & flowers4@1lnl.gov, 925-422-0529 \\
\hline Esther Ku & DOE & esther.ku@oak.doe.gov, 510-486-4297 \\
\hline Tony Leonard & Caltech & tony@ galcit.caltech.edu, 626-395-4465 \\
\hline Rose McCallen & LLNL & mccallen1@llnl.gov, 925-423-0958 \\
\hline Jerry Owens & LLNL & jlowens@Ilnl.gov, 925-422-1646 \\
\hline Jim Ross & NASA & jcross@mail.arc.nasa.gov, 650-604-6722 \\
\hline Jules Routbort & ANL/DOE & routbort@anl.gov, 630-252-5065 \\
\hline Walt Rutledge & SNL & whrutle@sandia.gov, 505-844-6548 \\
\hline Kambiz Salari & SNL & ksalari@sandia.gov, 505-844-9836 \\
\hline Ross Sheckler & Dynacs & dynacsny@dynacs.com, 315-626-6800 \\
\hline Ray Smith & LLNL & smith40@Ilnl.gov, 925-422-7802 \\
\hline Bruce Storms & NASA & bstorms@mail.arc.nasa.gov, 650-604-1356 \\
\hline Frank Tokarz & LLNL & tokarz1.llnl.gov, 925-423-3459 \\
\hline \multicolumn{3}{|c|}{ By Telephone } \\
\hline Adrian Tentner & ANL & tentner@anl.gov, 630-252-8454 \\
\hline Rich Valentine & ANL & richv@anl.gov, 630-252-4483 \\
\hline David Weber & ANL & weber@anl.gov, 630-252-8175 \\
\hline
\end{tabular}




\section{Agenda}

\section{Heavy Vehicle Aerodynamic Drag: Working Group Meeting \\ Lawrence Livermore National Laboratory \\ Livermore, CA}

Wednesday, March 16, 2000

Purpose of Meeting

Discussion of project technical and budget issues and proposed strategies to improve budget situation

Review of experimental and computational plans

Presentation of technical details of experimental and computational work in progress

Introduction to LLNL and Energy Directorates

Ray Smith

OHVT and budget

Jules Routbort

Introduction and issues

Rose McCallen

NASA data reduction/analysis/documentation and test plans

Jim Ross, Bruce Storms, Tom Arledge

USC test results/plans and a look at aero devices

Fred Browand

SNL RANS computations/analysis and DES development

Kambiz Salari, Walt Rutledge

Overview of TMC meeting

Frank Tokarz

LLNL LES compressible/incompressible computations/analysis

Tim Dunn, Dan Flowers, Jerry Owens, Rose McCallen

Caltech vortex method development and computations

Tony Leonard

Demonstration project: description and status

Ross Sheckler

Discussion:

Outline strategies/plans for resolving budget issues

Review computational/test plans for completeness

Discuss needed task expansions (e.g., design aspect) and research for project success

Decide on best collaborative approach with software industry and tractor manufacturers 


\title{
Aerodynamic Design of Heavy Vehicles
}

- Overview of Project

- Budget Issues

\author{
Rose McCallen
}

Lawrence Livermore National Laboratory, Livermore, CA

March 16, 2000 
At $70 \mathrm{mph}, 65 \%$ of the total energy expenditure is in overcoming aerodynamic drag.

Typical Class 8 tractor-trailer

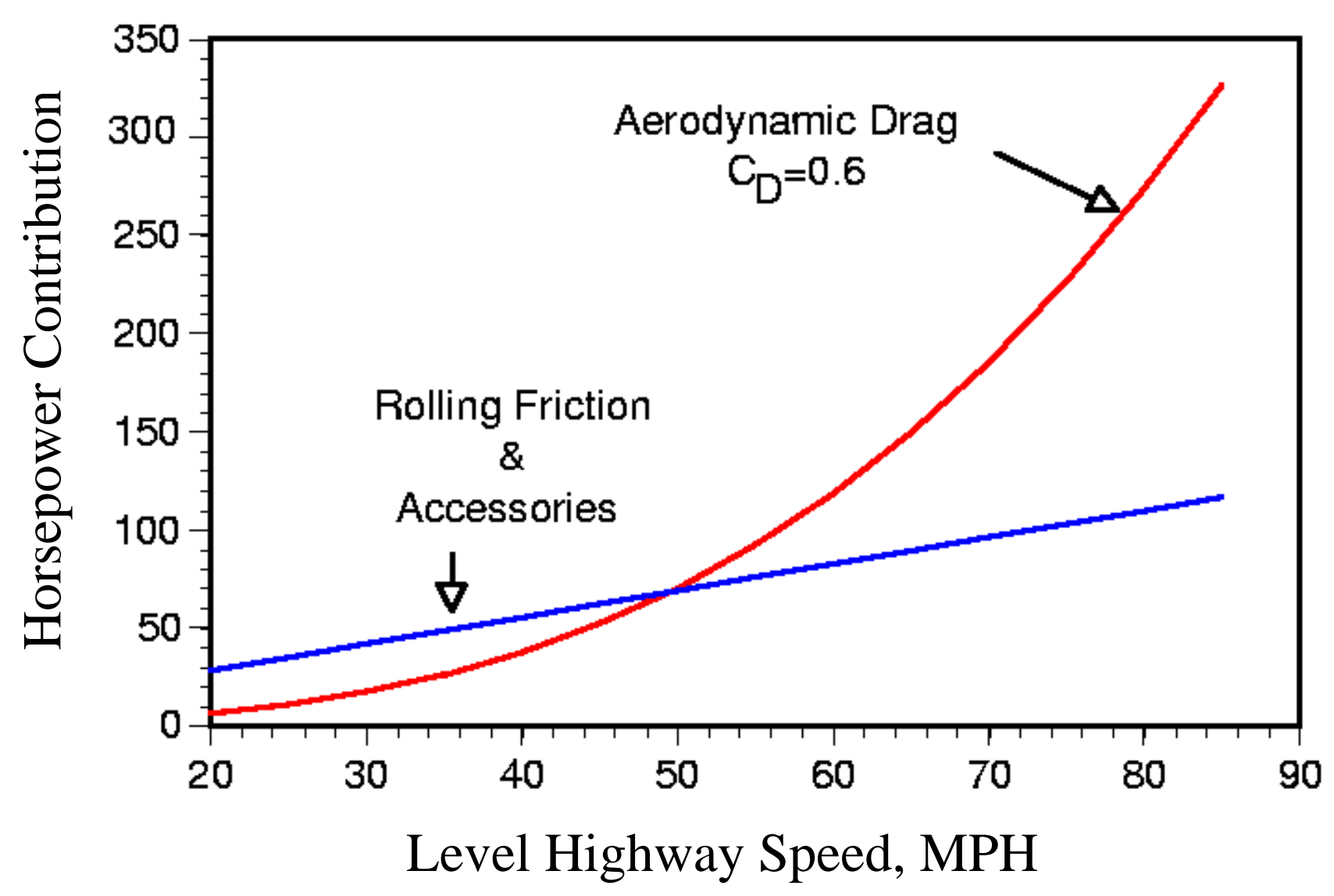


Reducing aerodynamic drag has a higher potential leverage than any other technology improvement.

\section{Year Projection}

\begin{tabular}{|l|c|}
\hline \multicolumn{1}{|c|}{ Technology } & Fuel Reduction \\
\hline Improve engine efficiency by $8 \%$ & $8 \%$ \\
\hline Weight reduction of $15 \%$ & $<10 \%$ \\
\hline Reduce aerodynamic drag by $25 \%$ & $10-15 \%$ \\
\hline
\end{tabular}

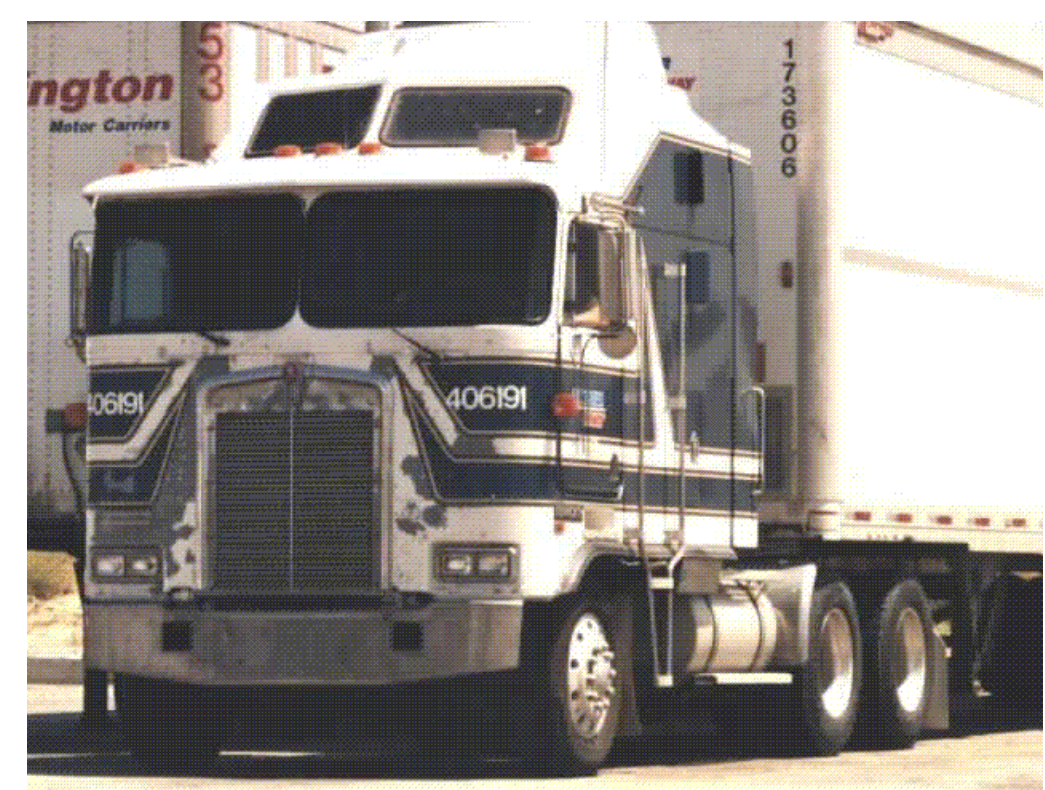

Kenworth cab-over-engine (1990)

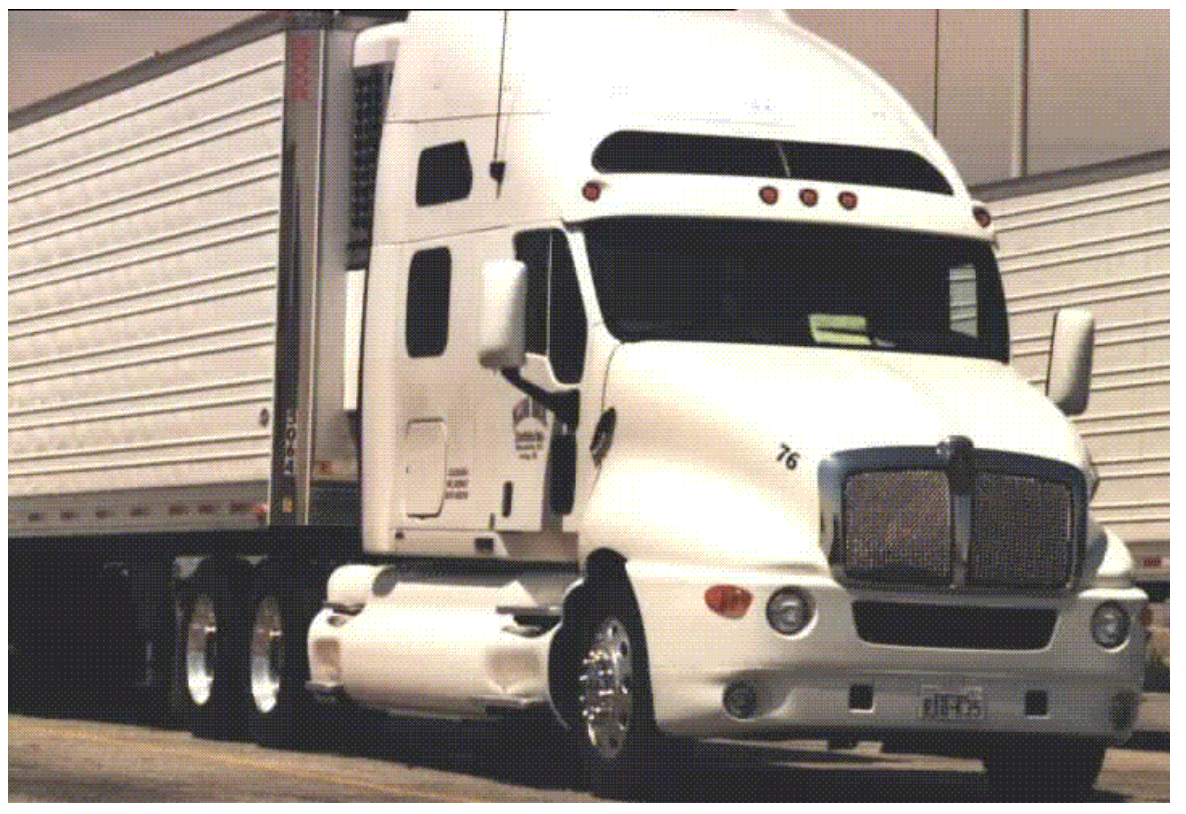

Kenworth T2000 conventional model (1999) 
The truck industry relies on wind tunnel and field experiments for aerodynamic design and analysis.

Wind Tunnel Testing

Costly detailed models

Expensive tunnel use

Trial-error approach to determine drag effects

Field Testing

Performed by both manufacturer and fleet operators

Issues

A tractor is paired with several different trailers

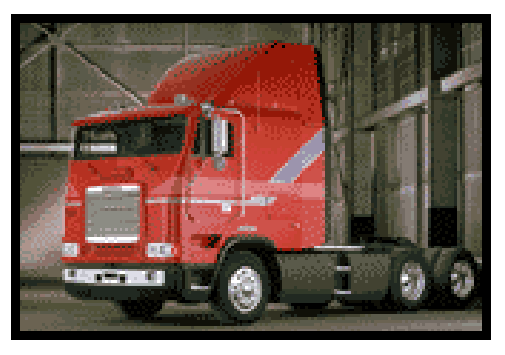

Cabover Engine

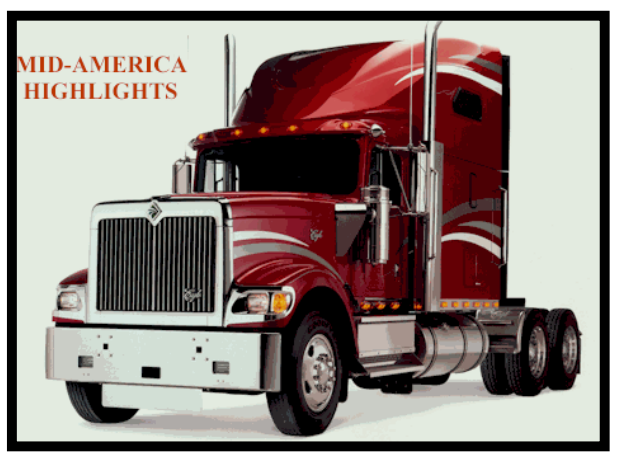

Conventional

Almost no aero design interaction between tractor and trailer manufacturers

The effects of design changes on drag are not well understood and computational guidance is needed 
The current project focus is on a validated simulation capability, but the MYPP includes a 'design' effort.

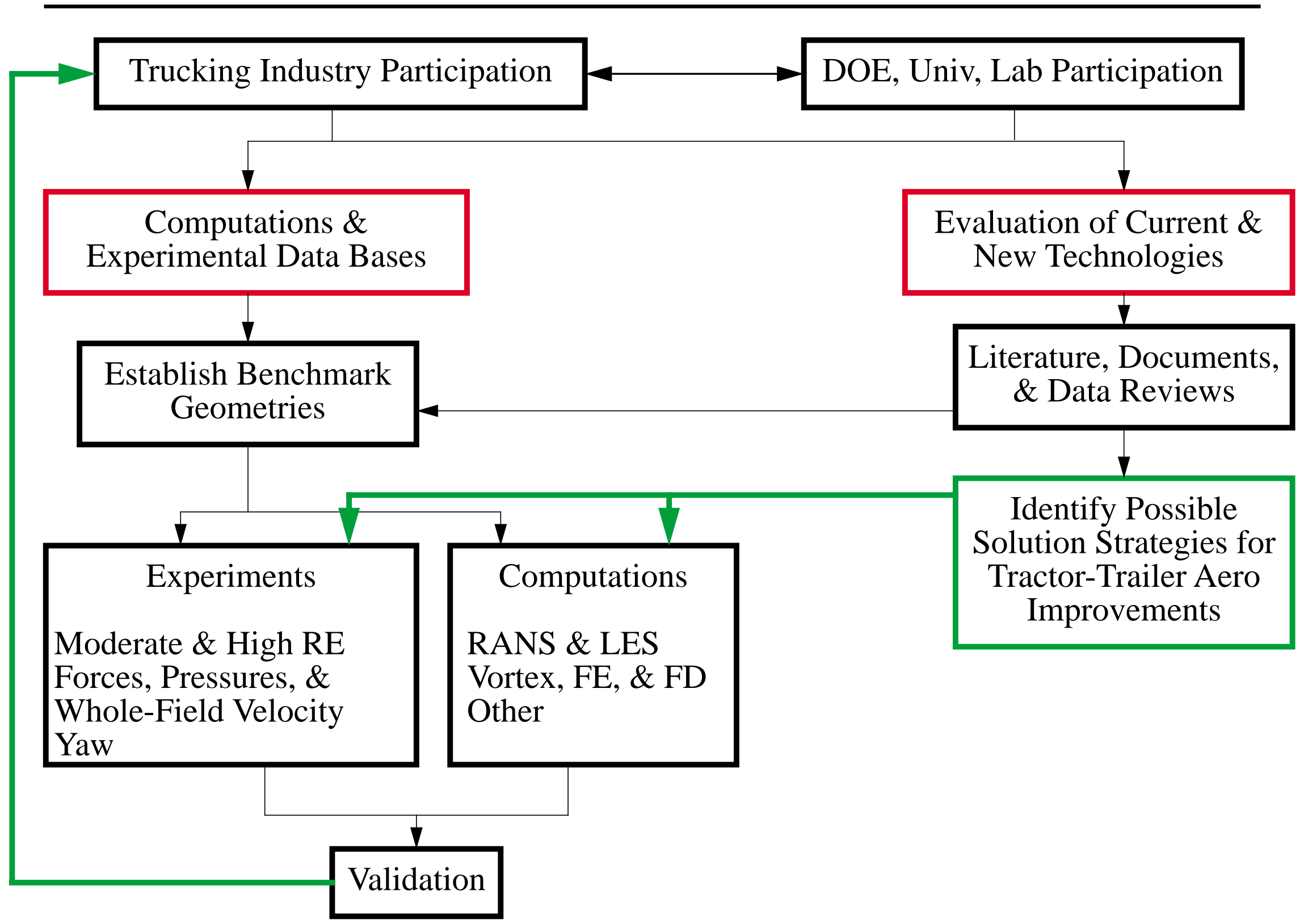


To obtain near-term results on a limited budget, we chose a simple geometry with existing data and modeling.

Ground Transportation System (GTS)
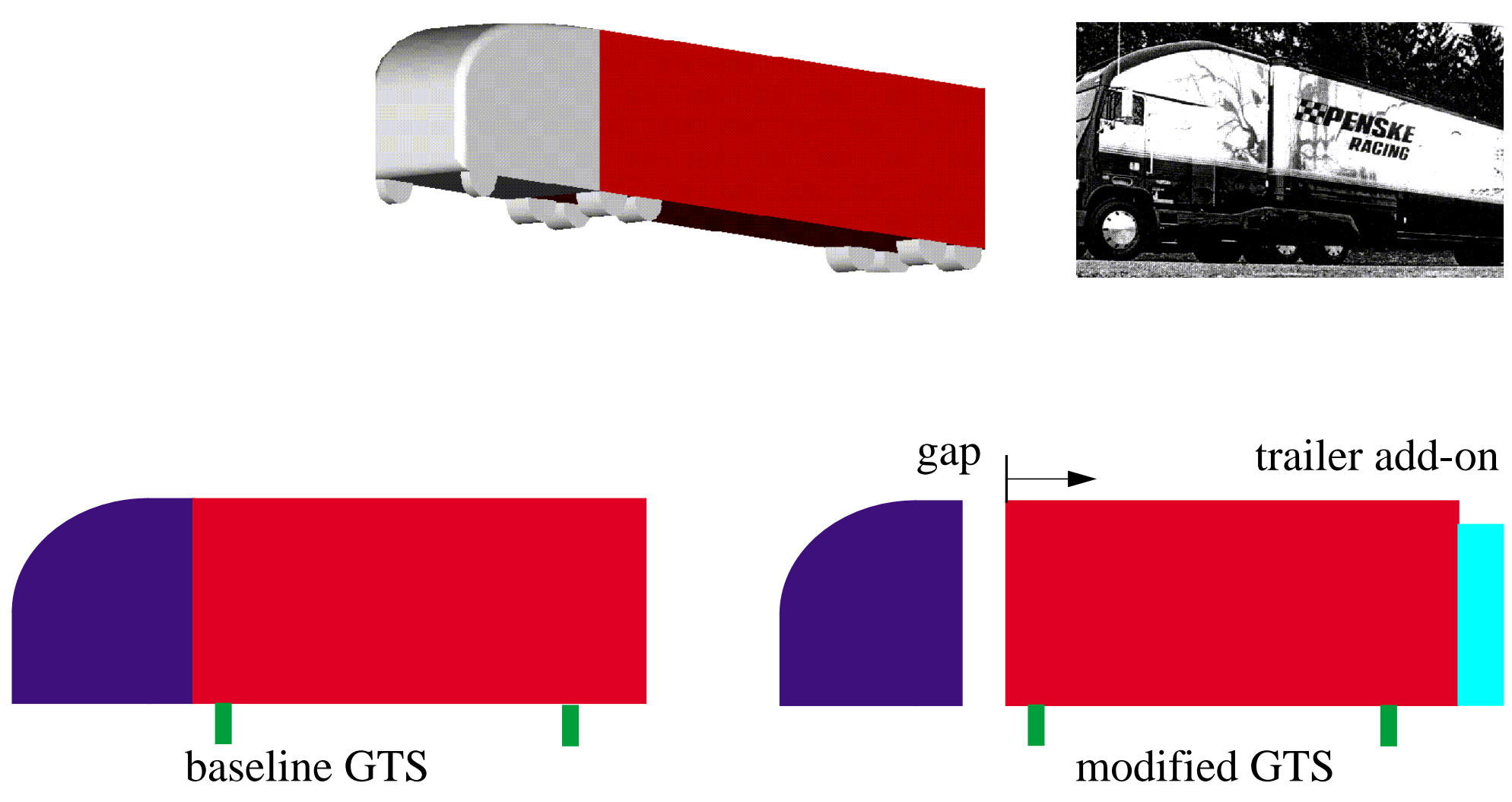
The DOE is interested in improved heavy vehicle thermal management for fuel reduction.

The engine cooling airflow contributes to aerodynamic drag

1970’s - 1980’s Designs

$\overline{\mathrm{C}}_{\text {Dtotal }}=1.0-0.85$

engine air cooling is $3.8 \%$ of $\overline{\mathrm{C}}_{\text {Dtotal }}$
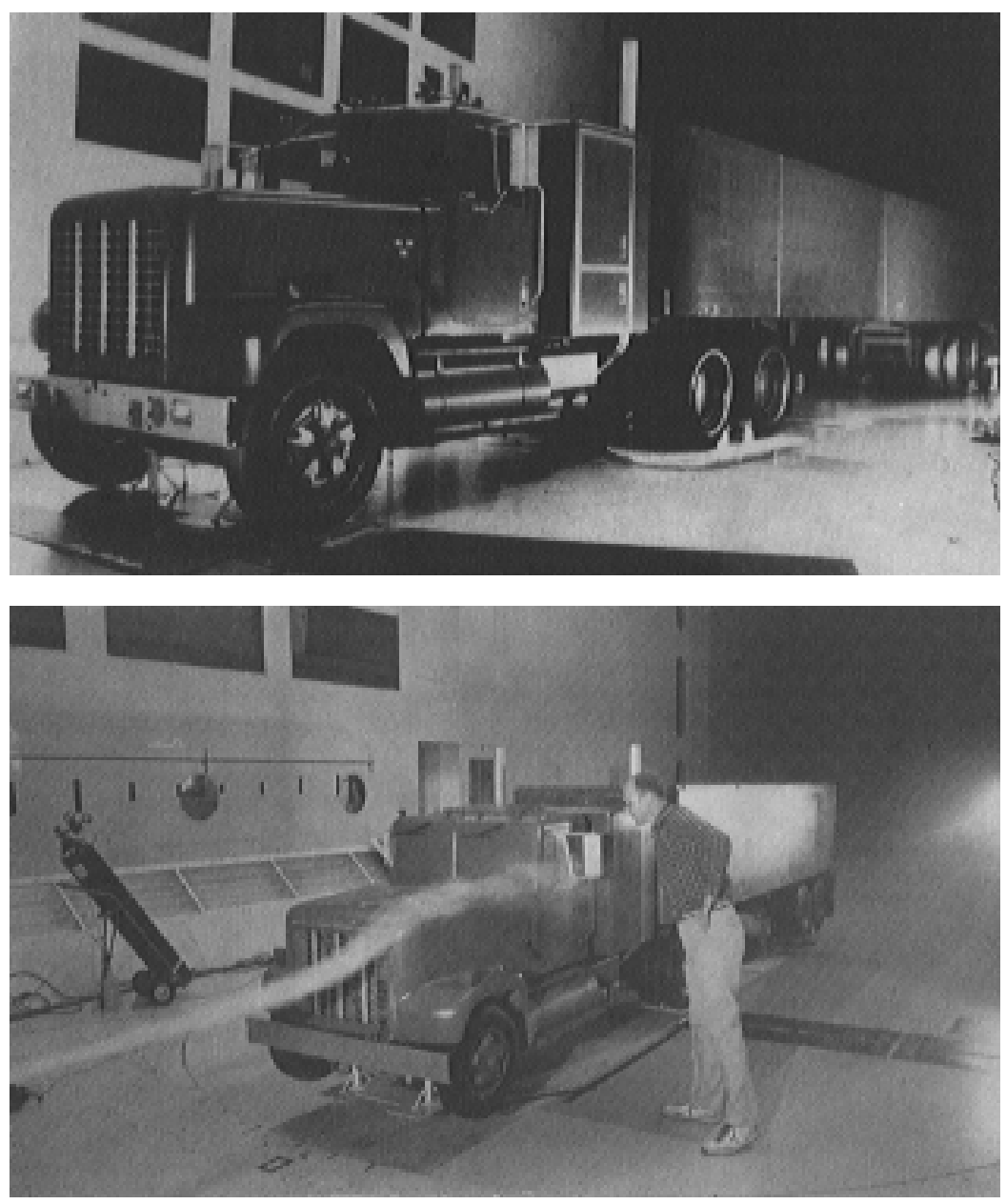


\section{Efficient aerodynamic design leads to less splash and spray.}

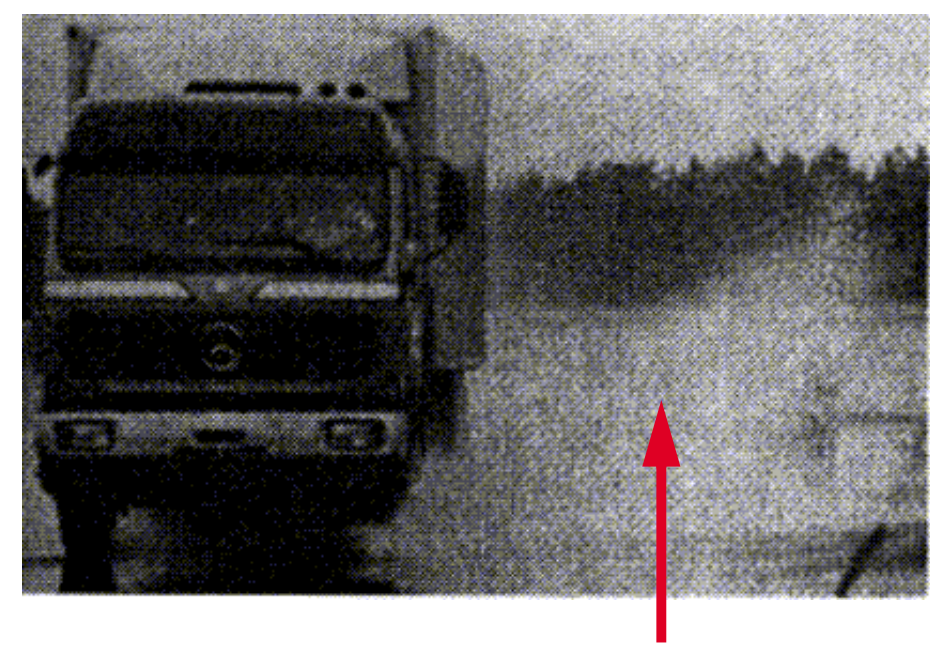

Car disappears behind water spray

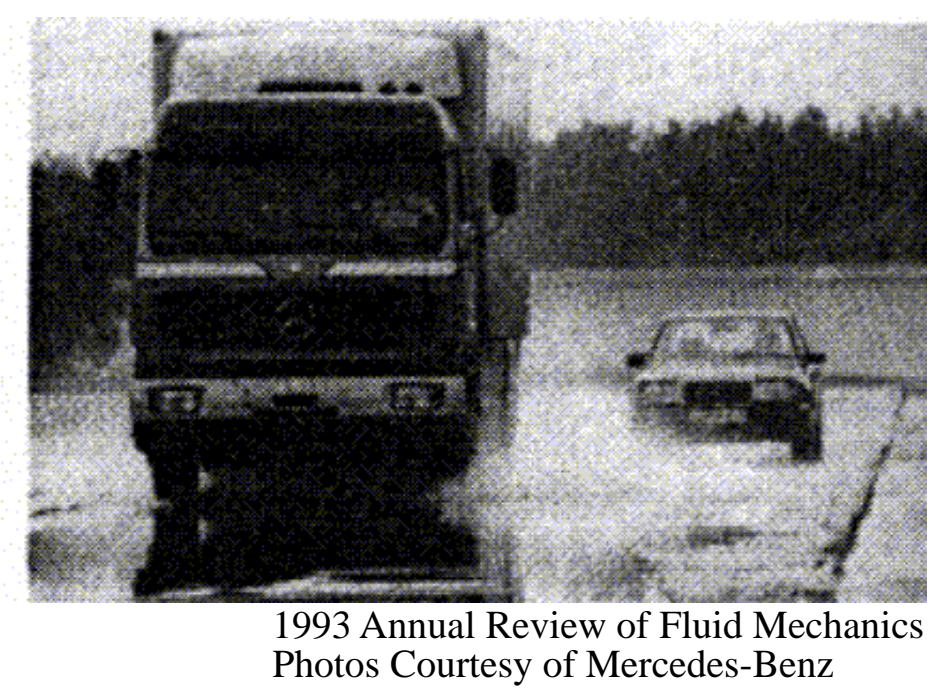

Grooved Mudguard reduced water flow between tire and mudguard

truck and car can be seen clearly truck not visib
to car driver

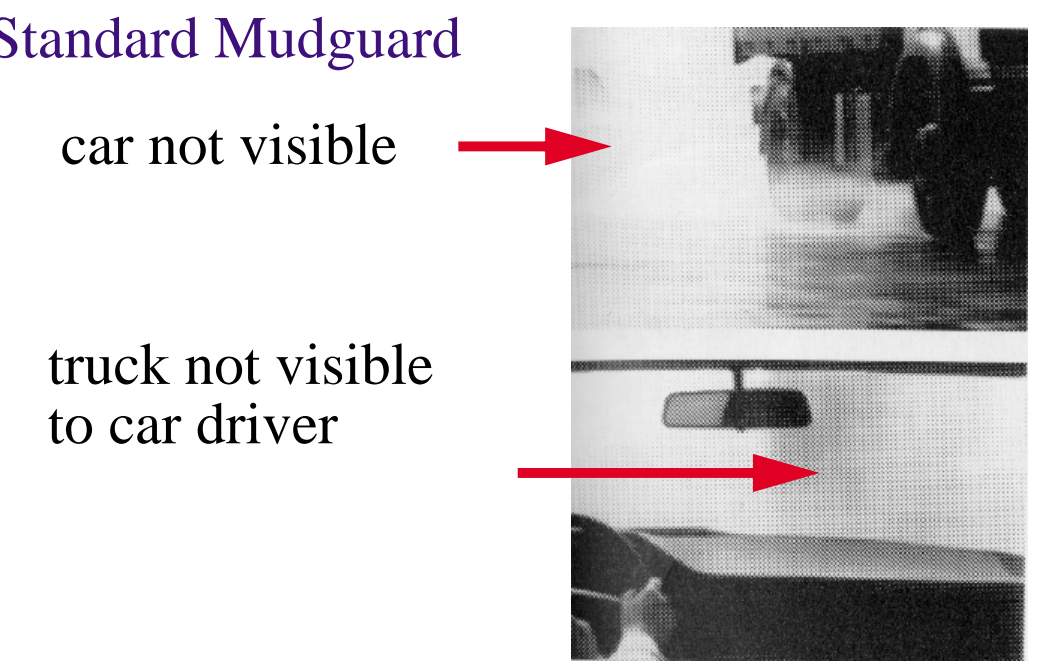

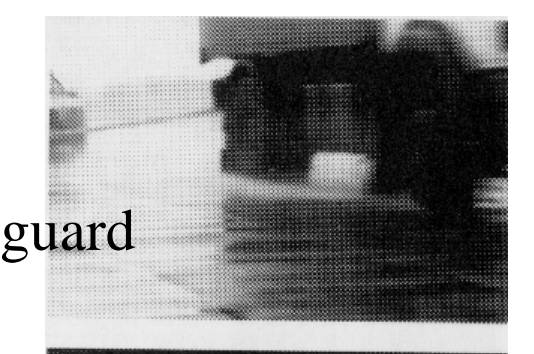

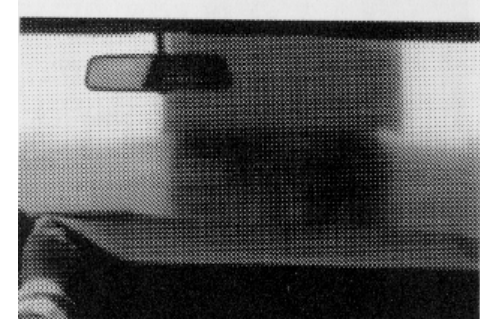

Ref. SAE paper 950631 


\section{Funding has been less than required.}

\begin{tabular}{|l|c|c|c|c|c|}
\hline & $\begin{array}{c}\text { Computations } \\
\& \text { Experiments }\end{array}$ & $\begin{array}{c}\text { Evaluation of } \\
\text { Current \& New } \\
\text { Technologies }\end{array}$ & Final Report & $\begin{array}{c}\text { Total requested/ } \\
\text { Year }\end{array}$ & $\begin{array}{c}\text { Total received/ } \\
\text { Year }\end{array}$ \\
\hline FY98 & $\$ 276 \mathrm{~K}$ & $\$ 34 \mathrm{~K}$ & & $\$ 310 \mathrm{~K}$ & $\$ 325 \mathrm{~K}$ \\
\hline FY99 & $\$ 630 \mathrm{~K}$ & $\$ 5 \mathrm{~K}$ & & $\$ 635 \mathrm{~K}$ & $\$ 441 \mathrm{~K}$ \\
\hline FY00 & $\$ 1,045 \mathrm{~K}$ & $\$ 188 \mathrm{~K}$ & & $\$ 1,233 \mathrm{~K}$ & $\$ 710 \mathrm{~K}$ \\
\hline FY01 & $\$ 1,095 \mathrm{~K}$ & $\$ 188 \mathrm{~K}$ & & $\$ 1,283 \mathrm{~K}$ & \\
\hline FY02 & $\$ 855 \mathrm{~K}$ & $\$ 161 \mathrm{~K}$ & & $\$ 1016 \mathrm{~K}$ & \\
\hline FY03 & $\$ 818 \mathrm{~K}$ & $\$ 161 \mathrm{~K}$ & & $\$ 979 \mathrm{~K}$ & \\
\hline FY04 & $\$ 120 \mathrm{~K}$ & $\$ 124 \mathrm{~K}$ & $\$ 34 \mathrm{~K}$ & $\$ 278 \mathrm{~K}$ & \\
\hline TOTAL & & & & $\$ 5,734 \mathrm{~K}$ & \\
\hline
\end{tabular}


Funding for FY98, FY99, and FY00

\begin{tabular}{|l|c|c|c|}
\hline & FY 98 & FY 99 & FY 00 \\
\hline LLNL & $\$ 100 \mathrm{~K}$ & $\$ 170 \mathrm{~K}$ & $\$ 210 \mathrm{~K}$ \\
\hline SNL & $\$ 100 \mathrm{~K}$ & $\$ 80 \mathrm{~K}+$ & $\$ 135 \mathrm{~K}$ \\
\hline USC & $\$ 50 \mathrm{~K}$ & $\$ 80 \mathrm{~K}$ & $\$ 100 \mathrm{~K}$ \\
\hline Caltech & $\$ 50 \mathrm{~K}$ & $\$ 80 \mathrm{~K}$ & $\$ 100 \mathrm{~K}$ \\
\hline NASA & $\$ 25 \mathrm{~K}$ & $\$ 31 \mathrm{~K}$ & $\$ 165 \mathrm{~K}$ \\
\hline & & & \\
\hline Totals & $\$ 325 \mathrm{~K}$ & $\$ 441 \mathrm{~K}$ & $\$ 710 \mathrm{~K}$ \\
\hline
\end{tabular}


It has been necessary to leverage other funding sources.

\begin{tabular}{|c|c|c|}
\hline \multirow[t]{4}{*}{ SNL } & - past data obtained at Texas A\&M & Free \\
\hline & - loan of model to NASA & Free \\
\hline & - LES/DES R\&D & LDRD, Tech Base \\
\hline & - computational resources and more & ASCI \\
\hline USC & - instrumentation & Caltrans, NSF \\
\hline \multirow[t]{2}{*}{ Caltech } & - LES model development & ASCI, DOD, internal \\
\hline & - computational resources & ASCI, NSF, DOD, internal \\
\hline \multirow[t]{3}{*}{ NASA Ames } & - 7’x10' wind tunnel tests & Free \\
\hline & - 12' wind tunnel tests & $1 / 3$ Cost \\
\hline & - loan of Navistar's model & Free \\
\hline \multirow[t]{2}{*}{ LLNL } & - computational resources & ASCI \\
\hline & - LES and code development & ASCI/Tech Base, other \\
\hline
\end{tabular}


Possible funding sources are being investigated.

DOE
Request for Proposals
Aerodynamic drag (LLNL, SNL, NASA, USC, Caltech)
Thermal management (ANL)
Friction and wear (ORNL)
Rolling resistance (ORNL)

DOD

Request for Proposals

Parasitic loss reduction

BES

$R \& D$ on simple shapes for turbulence model development 
We have a full calendar.

Progress Report (4/00)

DOE/EE/BES Proposal (4/00) - Assistance from OHVT needed

DOE RFP

Establish scope and industrial partners (WITHOUT TRAVEL)

Pre application (before 5/1/00)

Final proposal (before 5/15/00)

DOD RFP

Establish scope and partners - must be complimentary to DOE proposal

White paper $(2 / 29 / 00)$

Final proposal (before 4/28/00)

SAE Meeting, Washington, DC (6/00)

Abstract submitted

Draft paper $(3 / 15 / 00)$

Final paper and presentation

Working Group Meeting (7/00? Travel?)

Progress Report (7/00) 


\section{Accomplishments: The team of experts is established and significant progress has been made.}

- Established multi-lab, multi-university team

- Working relation with industry

- Multi-year program plan in place

- Experiments completed for baseline case (first time 3D, unsteady velocity field measured in a production wind tunnel)

- Understanding of gap flow phenomena

- Preliminary RANS and LES calculations

- Advanced model development in progress

- Continued data base development

- Preliminary design effort

\section{Baseline Case}

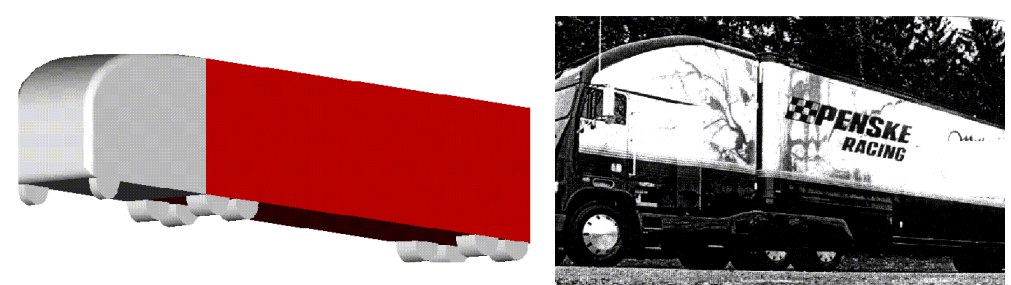

Experiments

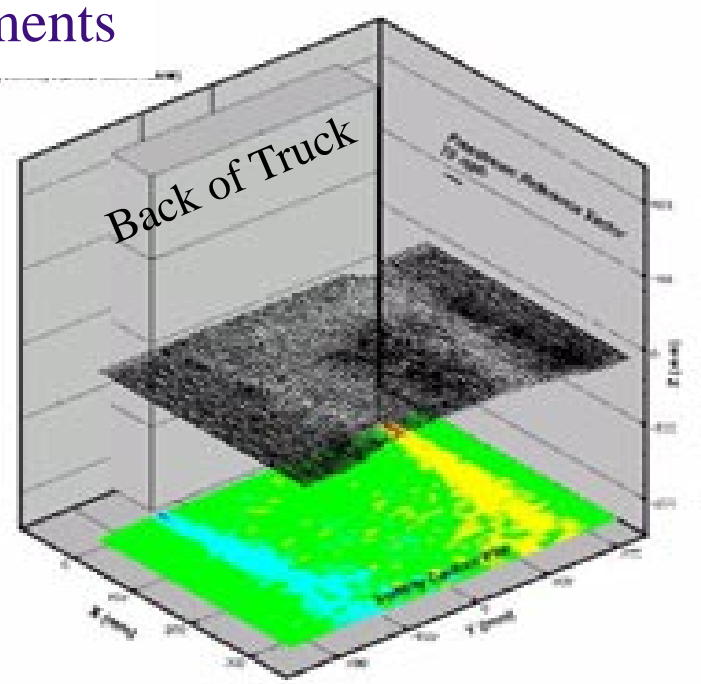

Computations

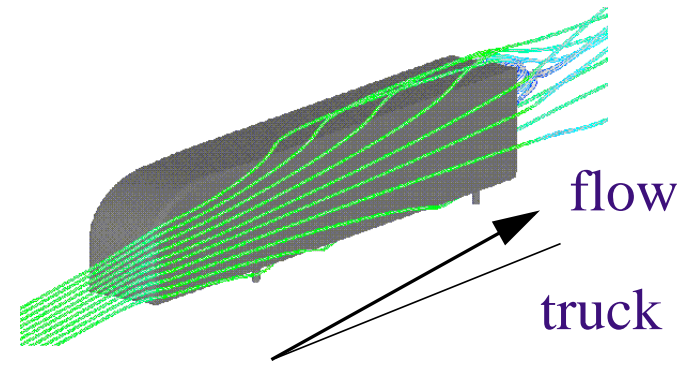




\section{$\frac{\text { Options for FY'00 Experiments at }}{\underline{\text { NASA ARC }}}$}

Two Possibilities and Associated Issues

- 12' Pressure Wind Tunnel

- Cost and schedule

- 7x10 Wind Tunnel

- Technical payoff 


\section{2’ Experiment Objectives}

- Re effect on wind-averaged drag

- Range from 0.5 to 5 million (based on width)

- Re effect on drag components - mirrors, bars, gap/side extenders, base-drag-reduction devices, etc.

- CFD validation data

- Skin friction

- Pressure distributions (PSP works great at high pressure)

- Unsteady pressures

- PIV not possible this year 


\section{Model for 12' Experiment Provided by Navistar}

- Navistar routinely tests to $140 \mathrm{mph}$ (50 psf) (at $60 \mathrm{mph}$, dynamic pressure is $9 \mathrm{psf}$ )

- Full-scale Re obtained in 12' at 6 atm (80 mph or q of 96 psf)

- Most model parts meet safety factor of 4 - those that don't can be replaced

- Balance arrangement like that used for America's Cup keel or fabricate new single- or two-axis balance (not an expensive proposition)

$\gg$ Max drag <400\#

$»$ Max side force < drag

- Yaw range of $\pm 14^{\circ}$ for wind averaging

- Generic model with CAD definition 


\section{2’ Issues}

- 3-week slot open in August - too soon to do the job right

- Cost is $\$ 160 \mathrm{~K} /$ week plus power

- Power $\sim \$ 10 \mathrm{~K} /$ week

- PIV not possible in this time frame

- Funding won't be available in time 


\section{Cost Breakdown for 12' Experiment}

What it takes to do the job right

\begin{tabular}{|c|c|c|c|c|c|}
\hline Item & Explanation & Cost basis & Per & Amount & Item cost \\
\hline Direct Labor & $\begin{array}{c}\text { Contracted labor research } \\
\text { engineer }\end{array}$ & $\$ 10,000$ & FTE month & 8.0 & $\$ 80,000$ \\
\hline Program Support & $\begin{array}{l}\text { Head tax for Experimental } \\
\text { Physics personnel }\end{array}$ & $\$ 40,000$ & FTE year & 1.5 & $\$ 60,000$ \\
\hline Wind Tunnel Time & 12' PWT charges & $\$ 160,000$ & week & 3.0 & $\$ 480,000$ \\
\hline Power Cost & $\begin{array}{c}\text { Cost of power to run wind } \\
\text { tunnel }\end{array}$ & $\$ 10,000$ & week & 3.0 & $\$ 30,000$ \\
\hline Model Mods & $\begin{array}{l}\text { Strengthen parts, balance } \\
\text { mods }\end{array}$ & $\$ 15,000$ & each & 1.0 & $\$ 15,000$ \\
\hline \multicolumn{6}{|l|}{ Instrumentation: } \\
\hline & PIV insertion optics & $\$ 30,000$ & each & 1.0 & $\$ 30,000$ \\
\hline & Laser installation & $\$ 15,000$ & each & 1.0 & $\$ 15,000$ \\
\hline & Camera housings & $\$ 10,000$ & each & 2.0 & $\$ 20,000$ \\
\hline & $\begin{array}{c}\text { Second laser for fwd } \\
\text { scatter }\end{array}$ & $\$ 40,000$ & each & 1.0 & $\$ 40,000$ \\
\hline & Seeder installation & $\$ 25,000$ & each & 1.0 & $\$ 25,000$ \\
\hline & PSP & $\$ 12,000$ & each & 1.0 & $\$ 12,000$ \\
\hline & & & & Instr. Total & $\$ 142,000$ \\
\hline Center taxes & $\begin{array}{c}\text { Directorate, Division, \& } \\
\text { Branch taxes }\end{array}$ & $3 \%$ & of net & $\$ 880,000$ & $\$ 26,400$ \\
\hline "Handling fee" & Tax on reimbursable $\$$ & $6 \%$ & of net & $\$ 880,000$ & $\$ 52,800$ \\
\hline & & & & Total & $\$ 886,200$ \\
\hline
\end{tabular}

Total for 2 week test \$716,200 


\section{Options for 7x10 Experiments}

- Full truck geometry from Navistar

- CFD validation data on full truck configuration » Probably without ugly details

- Additional base-drag reduction device demonstration

- Modified GTS model (Preferred option)

- Include gap/side extenders for higher-Re CFD validation

- Additional base-drag reduction device demonstration

- With either option, provide opportunity for tests of USC model at lower blockage and higher Re 


\section{Proposed Mods to GTS Model}

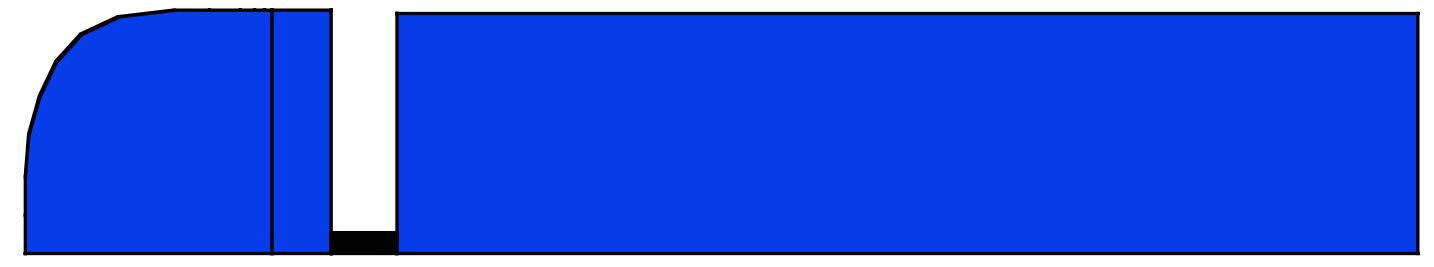

Side view

Cut gap in model sized appropriately for long-haul trucks

Instrument gap and side extenders to measure mean and fluctuating pressure

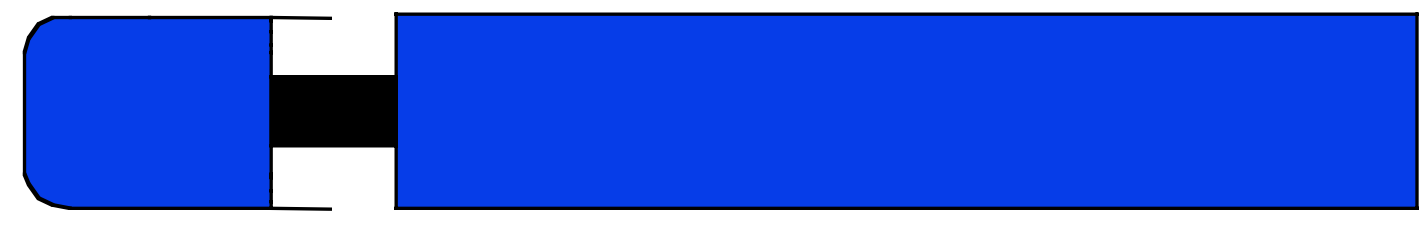

Top view

Side extenders to model cavity on trucks

- transparent set to allow PIV measurements

- instrumented set for mean and unsteady pressure

Fabricate set of plates to allow restoring model to GTS configuration 


\section{Aeroplate $^{\mathrm{TM}}$ Concept}

- Developed by B.L.A. Technologies
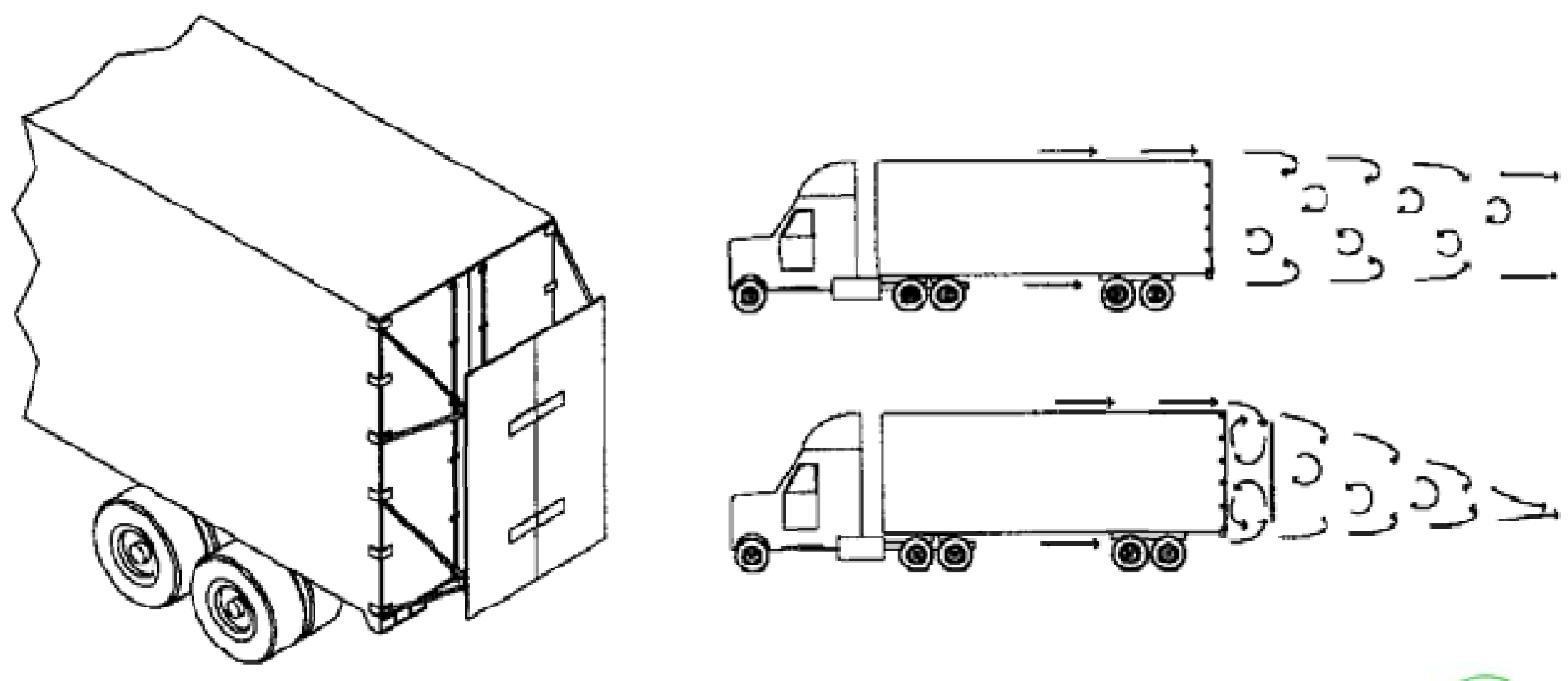


\section{Aeroplate $^{\mathrm{TM}}$ Folding}
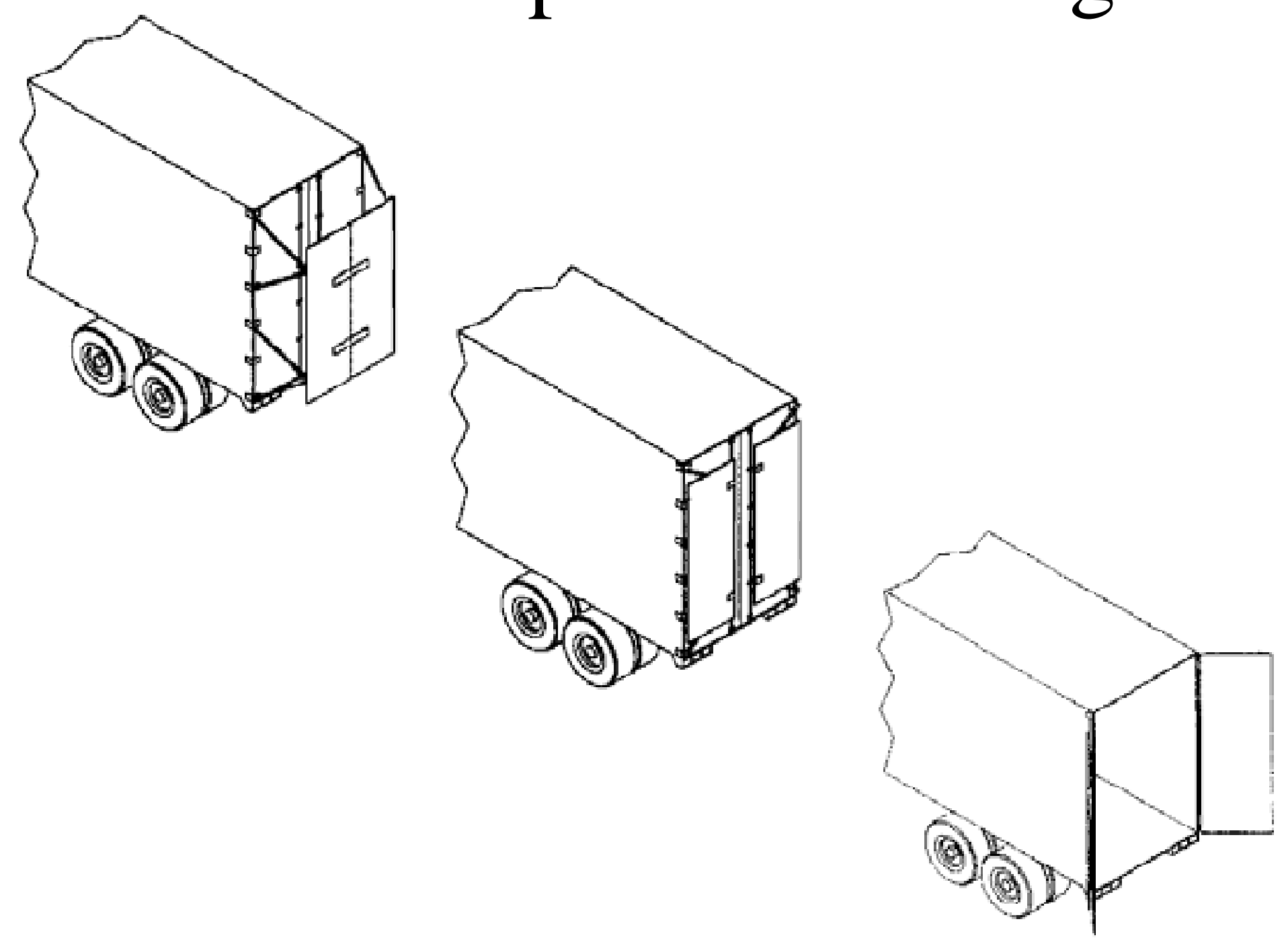

Experimental Physics Branch 


\section{Proposed Test Matrix}

- Side extender variations for drag (3 or 4 )

- Select 2 extenders for PIV and other detailed measurements

- At least 2 yaw angles

- Repeat drag data for baseline GTS

- Examine drag reduction with and without gap for

- CDI boat-tail plates

- BLA drag plate

- Other drag-reduction devices?

- If noticeable difference in drag reduction with gap, perform PIV measurements in wake to diagnose 


\section{Measurements}

- Pressure distributions (no PSP unless big demand)

- Oil film skin friction (limited configurations)

- 3-D PIV in gap (and possibly in wake)

- Mean and unsteady pressures in gap area and rear door

- Need to define measurement requirements for USC model 


\section{Proposed CFD Work at Ames}

- Overflow computations of geometry with gap \& side extenders at 2 yaw angles and $2 \mathrm{Re}$

- 2 month effort

- Donovan Mathias will do work - running ship air wake computations for Navy

- Similar to Navy Ship Airwake computations currently underway 


\section{Sample Effort \#1:}

\section{LHA/LHD Vortex Visualization}

(All cases 340 deg WOD; vortex core located near port deck edge)
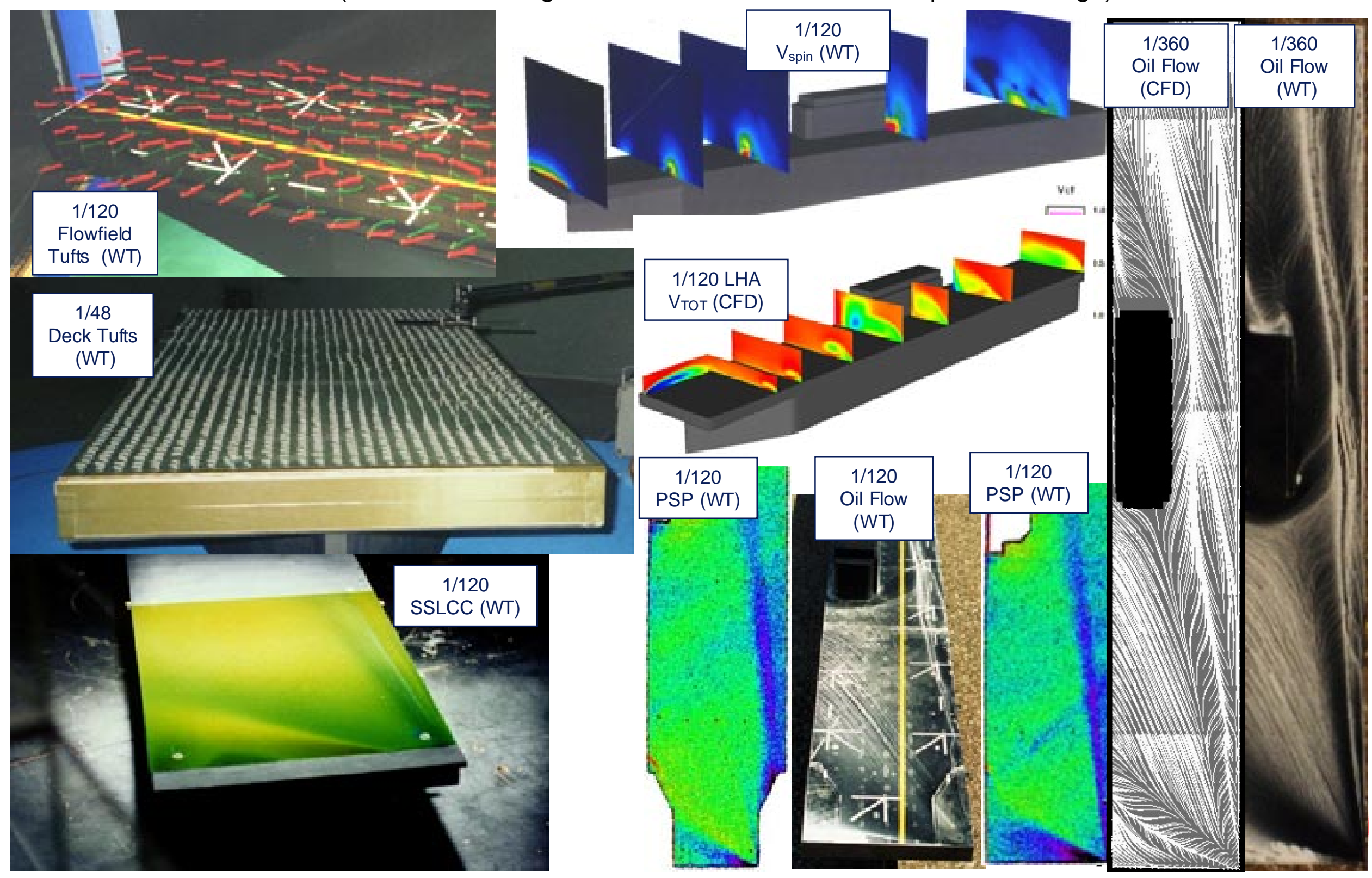


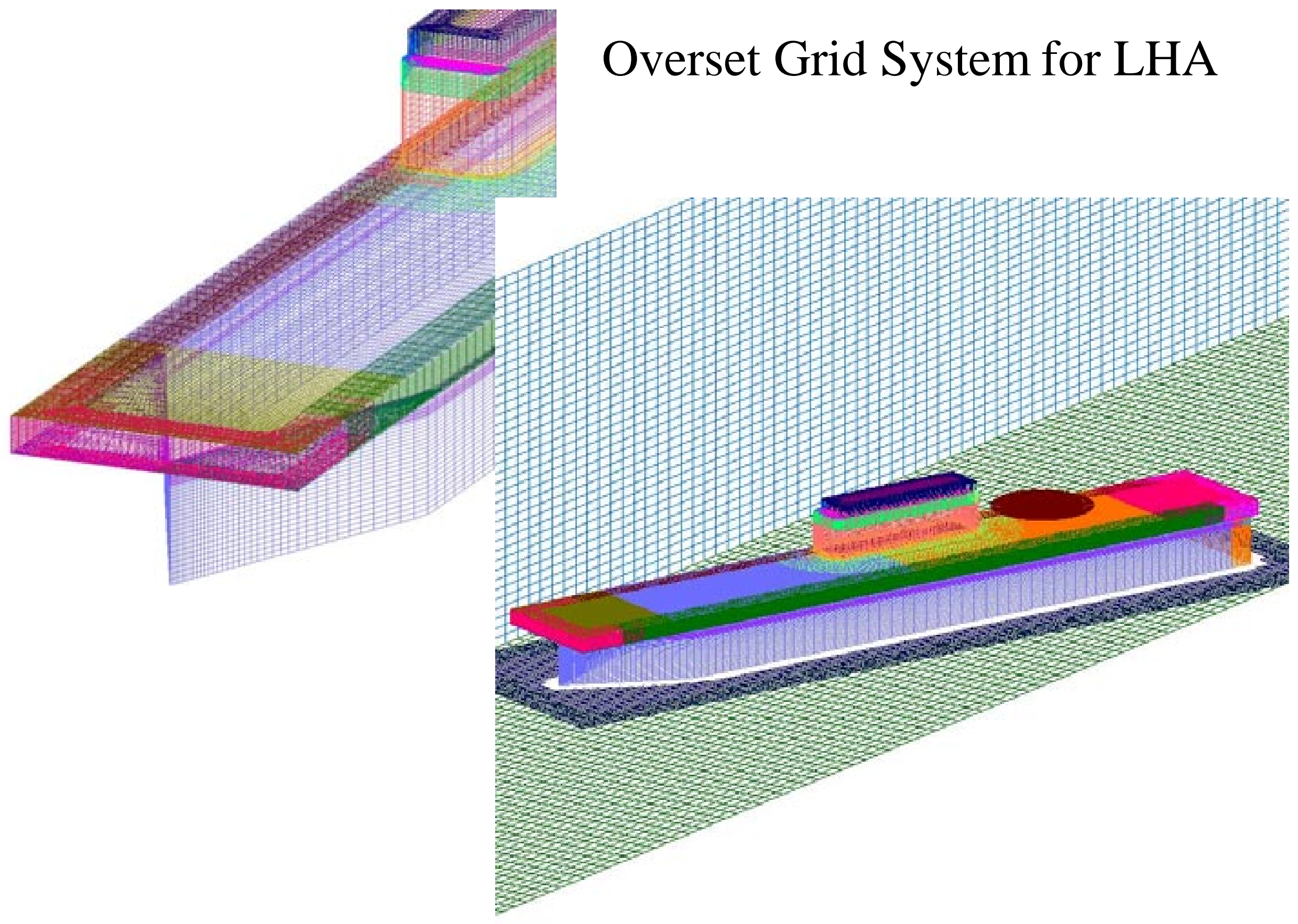




\section{Effect of Hovering H-60 Rotor Thrust \\ on Future LHA ${ }^{1}$ Flow Patterns}

(Structured RANS CFD Solutions by D. Mathias, 340 deg winds)

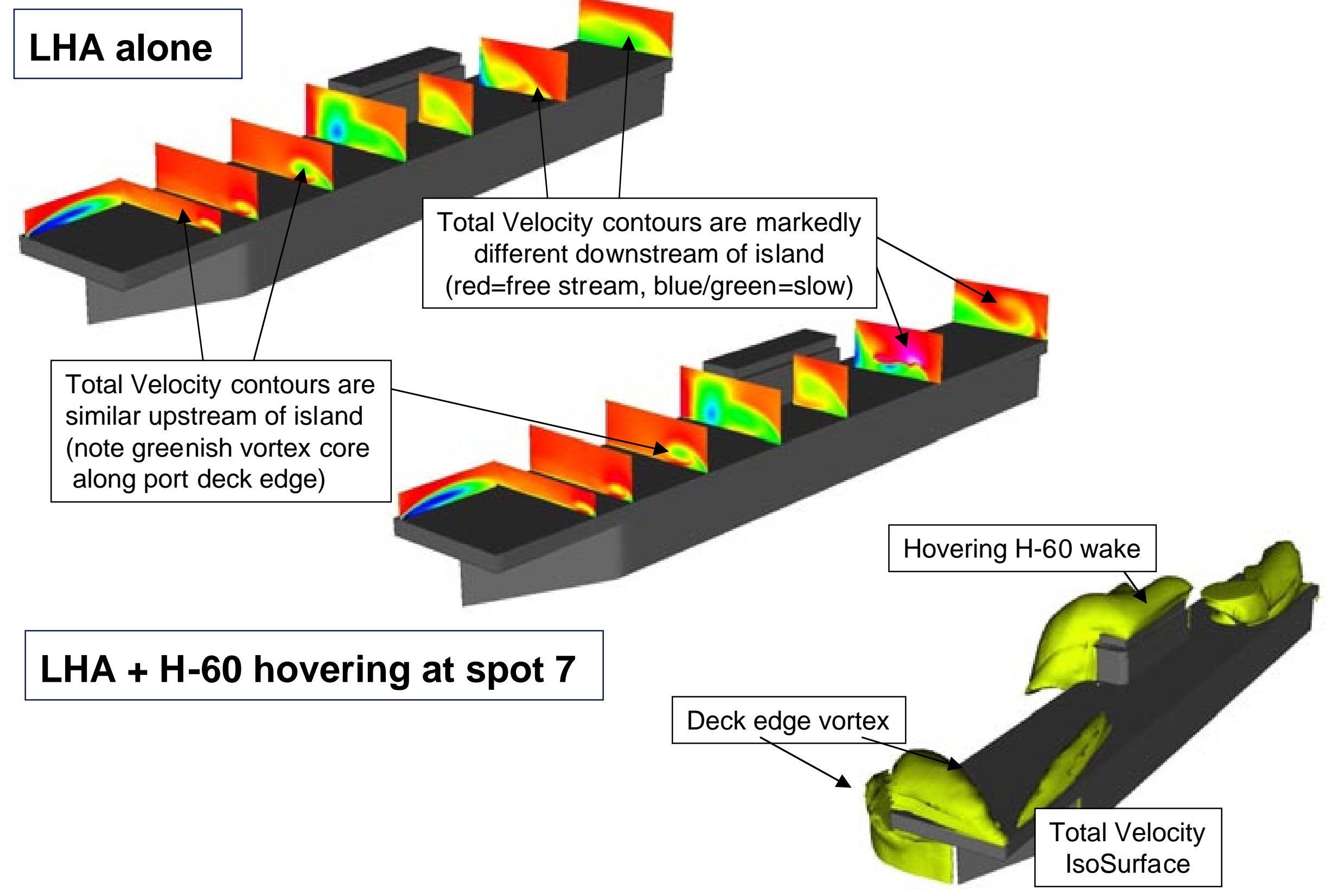




\section{Ames Activities for FY '01}

- No real need for additional testing at low Re

- Experimental work at Ames should be high-Re only

- Maybe not plan any experiments for next year unless

12 ' experiment can be funded

- Overset grid computations? 


\section{USC Results/Plans}

Fred Browand/Mustapha Hammache

\section{Resolving Differences in Measured Drag}

Coefficients

Tractor-Trailer Gap Flow at Yaw

Modification of TrailerBase Flow by Means of Oscillatory Perturbations 


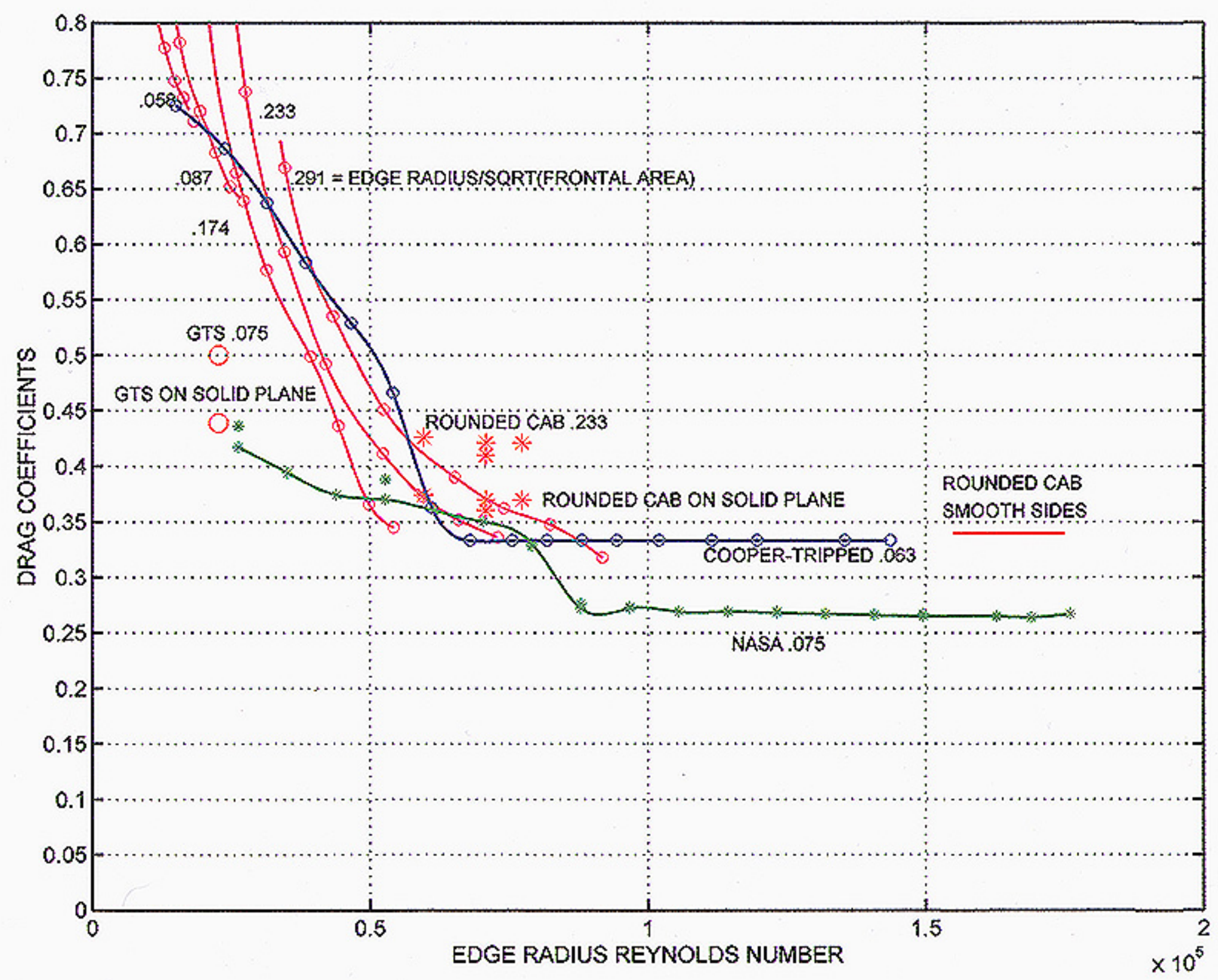




\title{
Computational Prediction for a Simplified Truck Geometry
}

\author{
Kambiz Salari \\ Walter H. Rutledge \\ Aerosciences and Compressible Fluid Mechanics Department \\ Sandia National Laboratories \\ Heavy Vehicle Aerodynamic Drag: Working Group Meeting \\ Lawrence Livermore National Laboratory
}

March 16, 2000 


\section{Outline}

- Sandia computational approach

- Ongoing Sandia simulations

- Leveraging from other programs

- Modeling wind tunnel experiment

- Tunnel empty flow simulation

- Flow quality in the tunnel test section

- Results for $0^{\circ}$ and $10^{\circ}$ yaw

- Conclusions 


\section{Sandia Computational Approach}

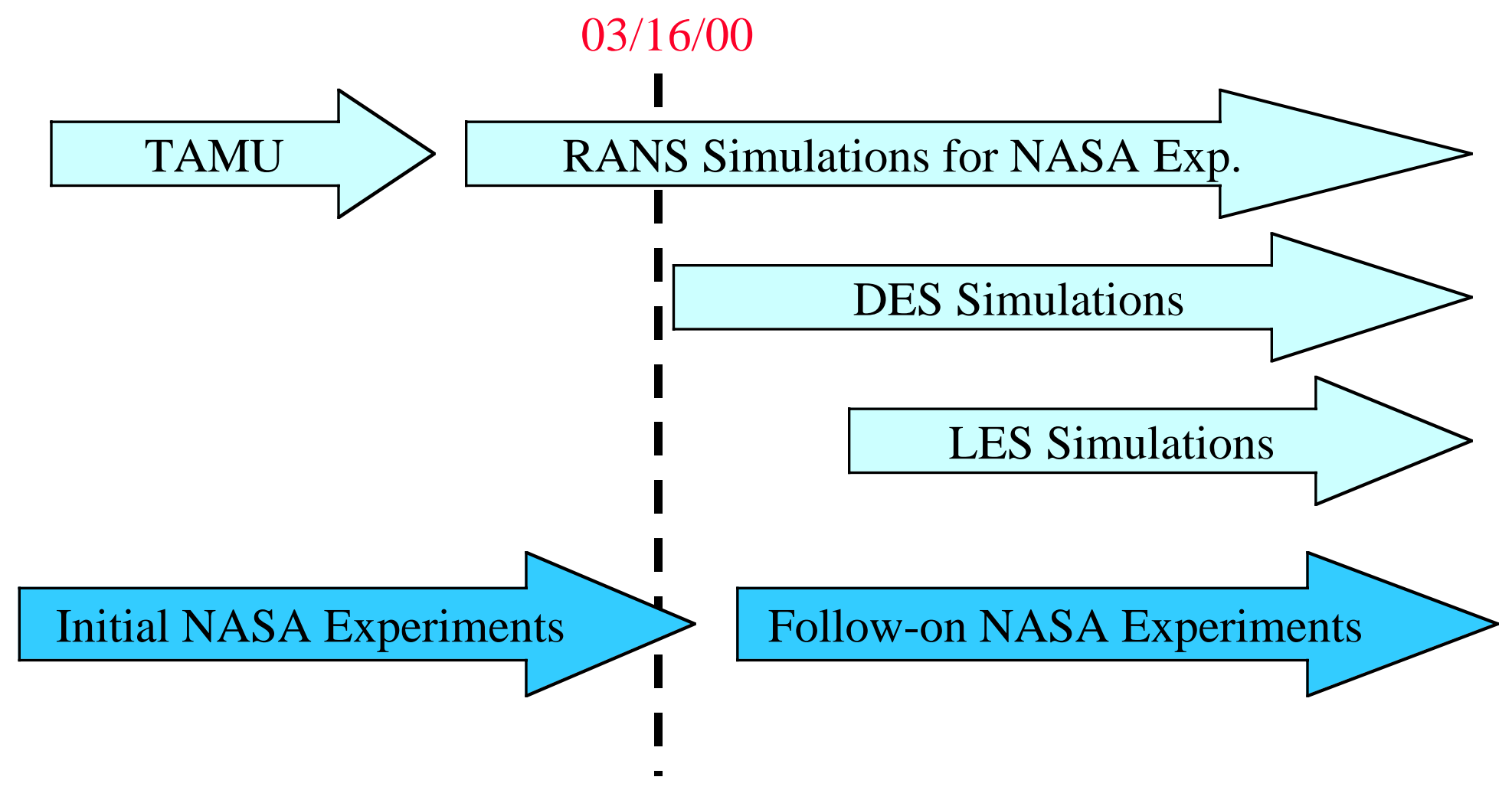




\section{Ongoing Sandia Simulations}

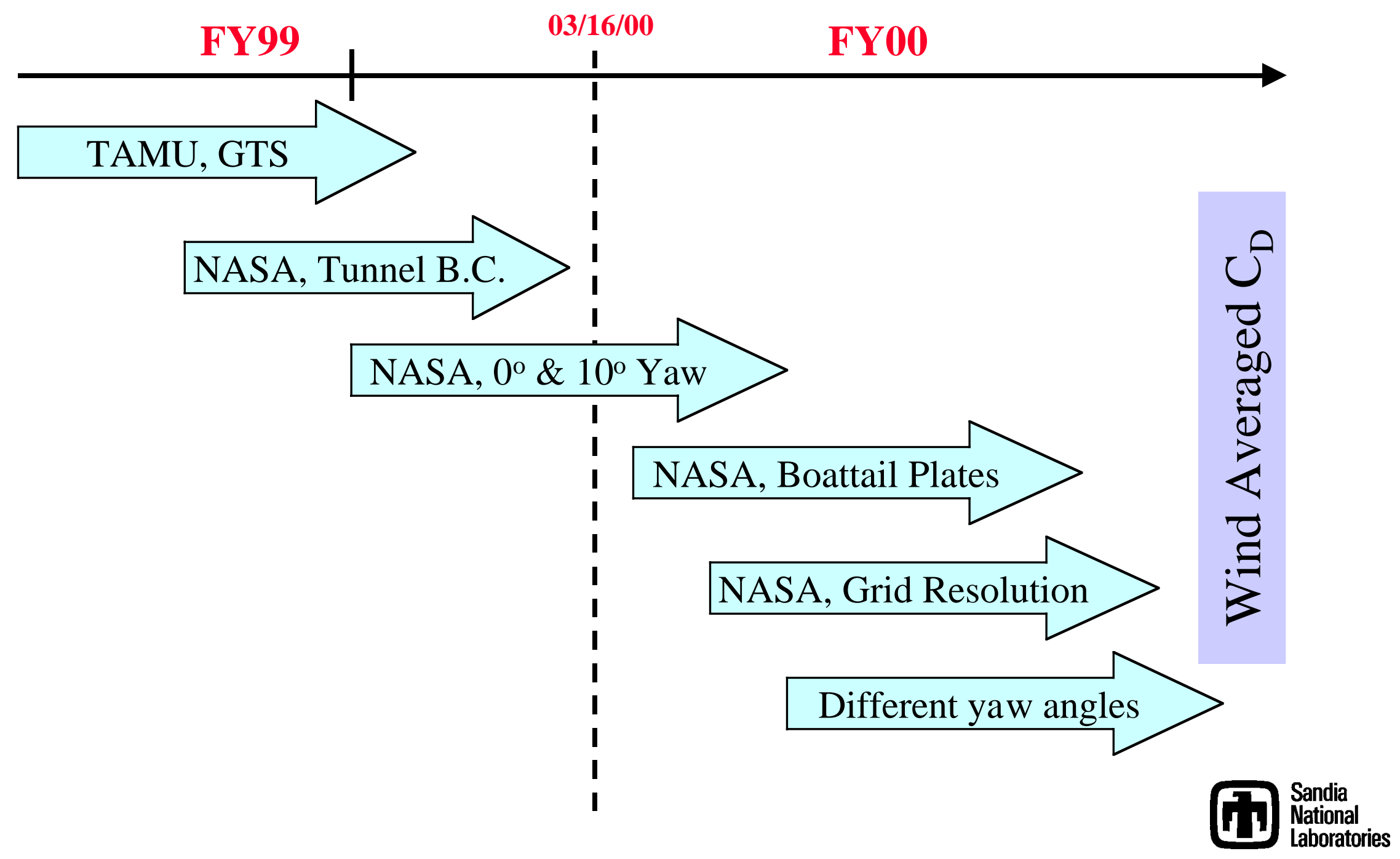




\section{Leveraging from Other R\&D Programs}

- DOE Accelerated Strategic Computing Initiative (ASCI) Aerodynamics program

- RANS Code Development (SACCARA)

- Intel Teraflop access time

- Verification \& Validation Activities

- Sandia Engineering Sciences Research Foundation (Tech Base, LDRD)

- Transition and Turbulence Modeling

- DES Research

- Potential Industrial CRADA

- LES Development \& Application 
The Six Recommended Practices of a "Validation Experiment"

- Validation experiment should be jointly designed and executed by experimentalists and code developers

- Validation experiments should be designed to capture the relevant physics, all initial and boundary conditions and auxiliary data (viscosity, flow rates, etc.)

- Validation experiments should utilize any inherent synergisms between experiments and computational approaches (try to offset strengths and weaknesses between the two) 


\section{The Six Recommended Practices of a "Validation Experiment" (cont'd)}

- The flavor of a blind comparison of computational results with experimental data should be a goal (that is, it should be an attempted to be a true prediction not a code calibration)

- Level of complexity of physics should be attacked in a series of validation experiments (start off simple with experiments at high confidence and work up to more complex flows, e.g., turbulent flows) 
The Six Recommended Practices of a "Validation Experiment" (cont'd)

- Develop and employ experimental uncertainty analysis procedures to delineate and quantify systematic and random sources of errors

Reference: Oberkampf and Aeschliman, AIAA Journal, May 1998, pp. 733-741.

Reference: AIAA Guide to Verification and Validation of Computational Fluid Dynamics 


\section{Modeling Wind Tunnel Experiment}

\section{Computational Boundary Conditions}

- Inflow

- Boundary layer profile

- Uniformity of the incoming flow

- Description of turbulent fluctuations (intensities)

- Outflow

- Modeling tunnel walls (blockage) 


\section{NASA 7'x10' Tunnel, Flow Simulation}

Test Condition for run 7:

Total pressure $=102,652.76\left(\mathrm{~N} / \mathrm{m}^{2}\right)$

Static pressure $=97,612.51\left(\mathrm{~N} / \mathrm{m}^{2}\right)^{\star}$

Dynamic pressure $(Q)=5,040.24\left(\mathrm{~N} / \mathrm{m}^{2}\right)$

Static Temperature $=5^{\circ} \mathrm{C}$

Mach number $=0.27$

Yaw angle $=0^{\circ}$ and $10^{\circ}$

$R e=2 \times 10^{6}$ (based on truck width)

"Based on equivalent "Tunnel Empty" Condition 


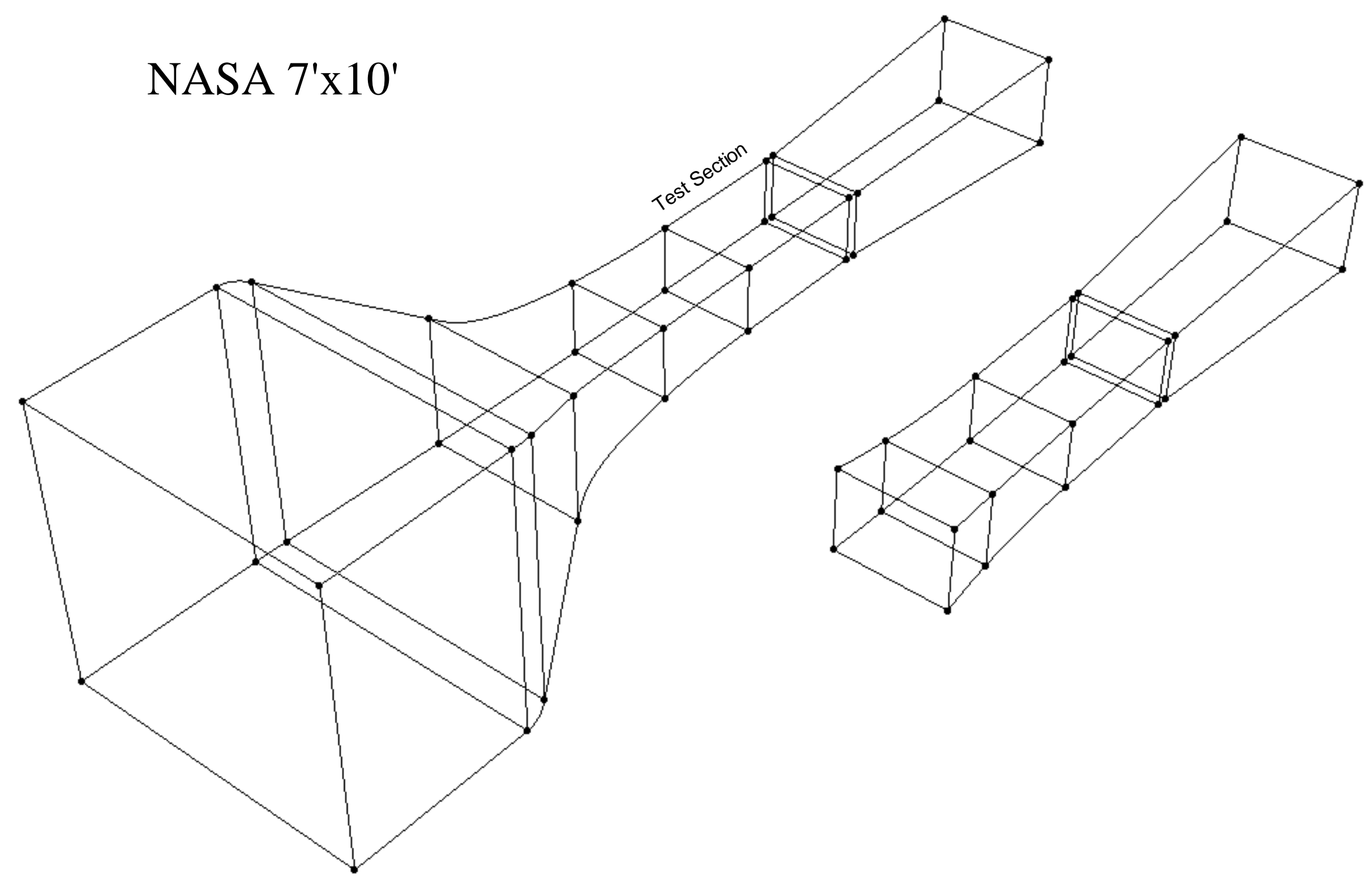




\section{NASA 7'x10' Tunnel, Flow Simulation}

Pressure contours

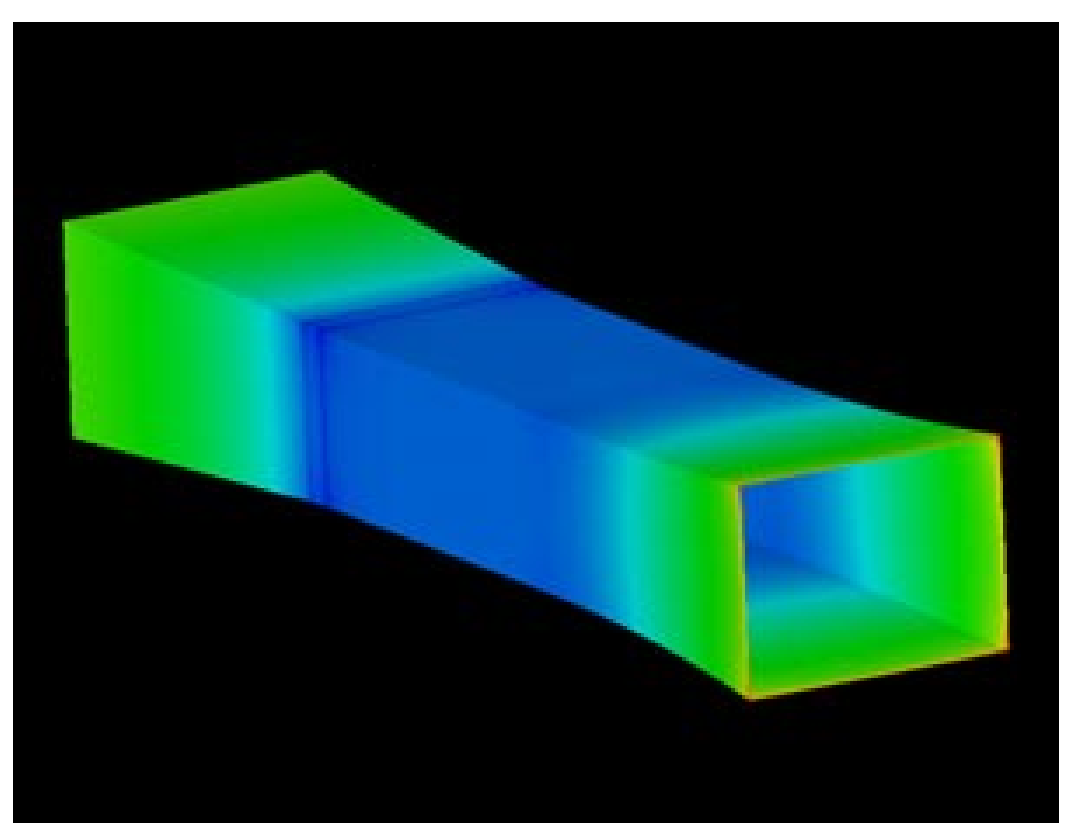

Mach contours (centerline)

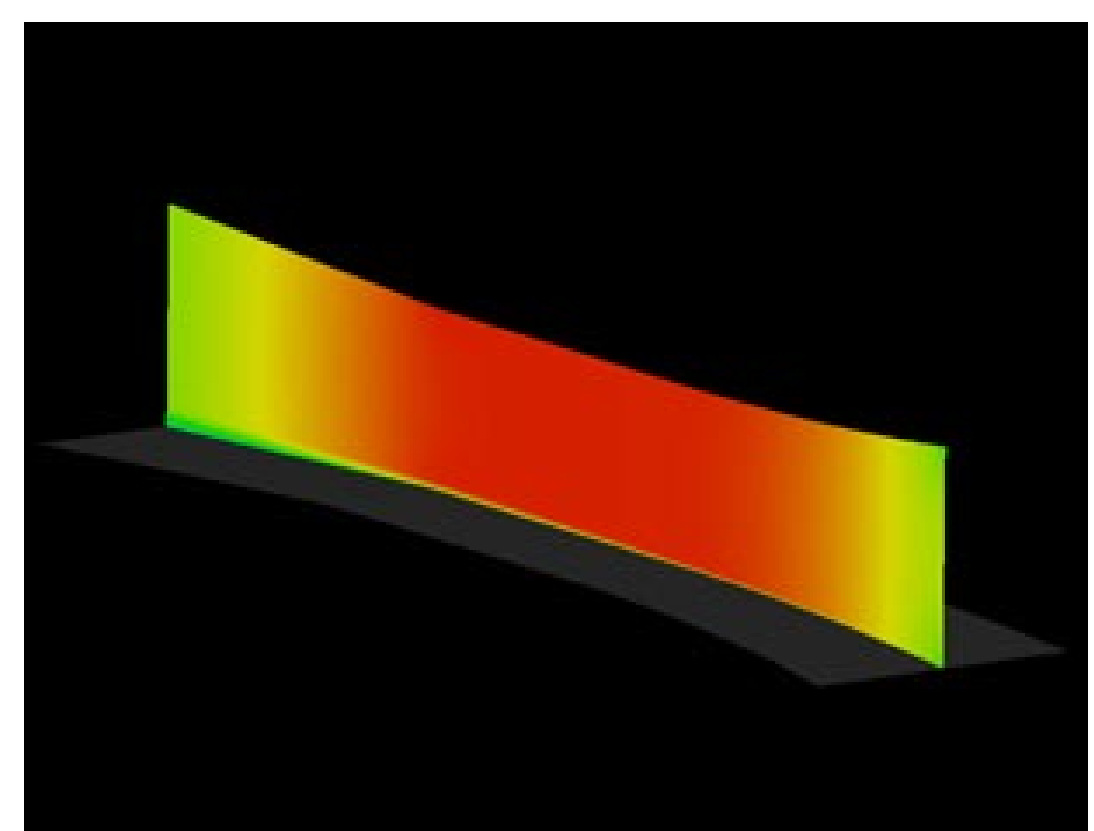




\section{NASA $7^{\prime} \times 10^{\prime}$ Tunnel, Flow Simulation, ...}

\section{Iso-Surface Plots}

u-component, $\mathrm{u}=89.92 \mathrm{~m} / \mathrm{s}$

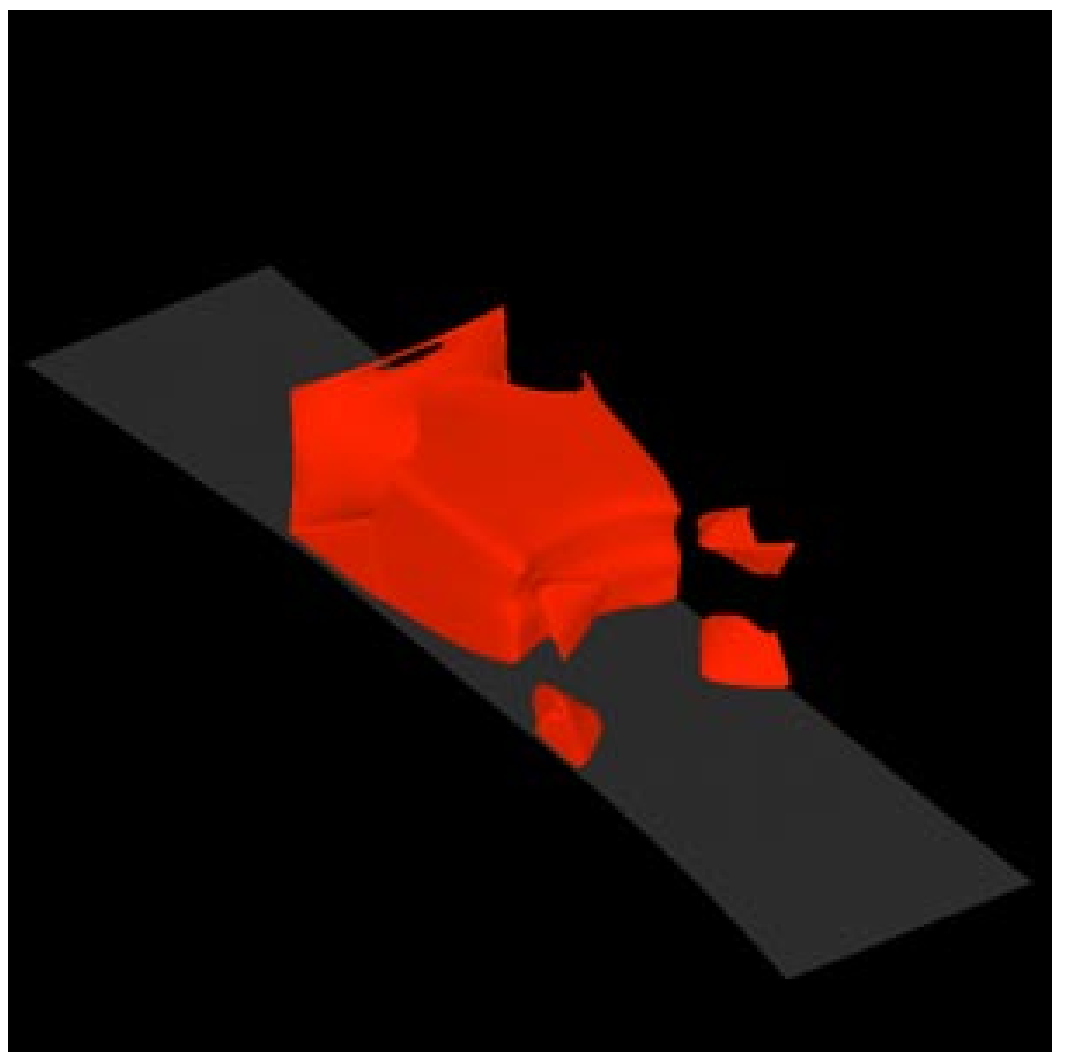

v-component, $\mathrm{v}=0.1 \mathrm{~m} / \mathrm{s}$

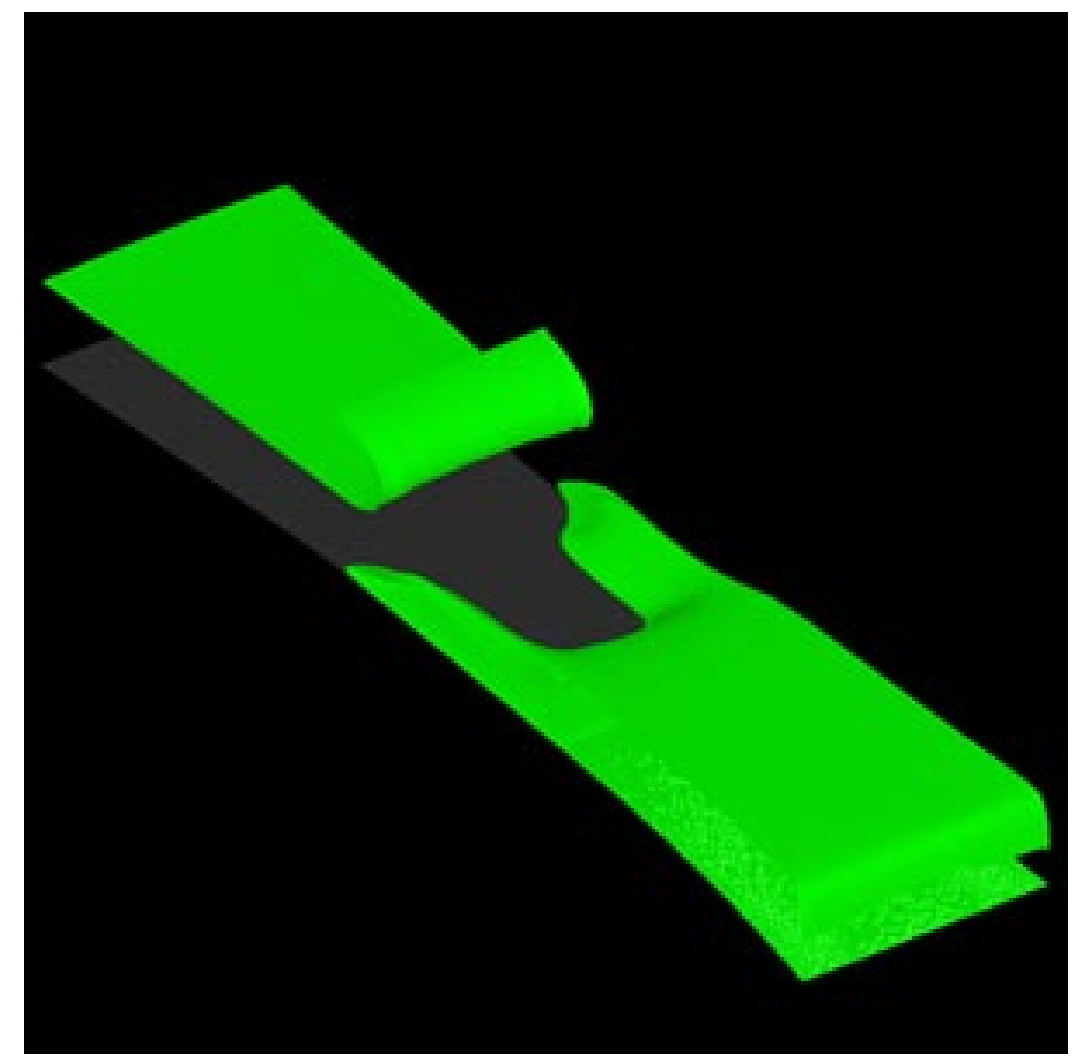




\section{NASA $7^{\prime} \times 10^{\prime}$ Tunnel, Flow Simulation, ...}

\section{Iso-Surface Plots}

$\mathrm{W}$-component, $\mathrm{w}=-0.01 \mathrm{~m} / \mathrm{s}$

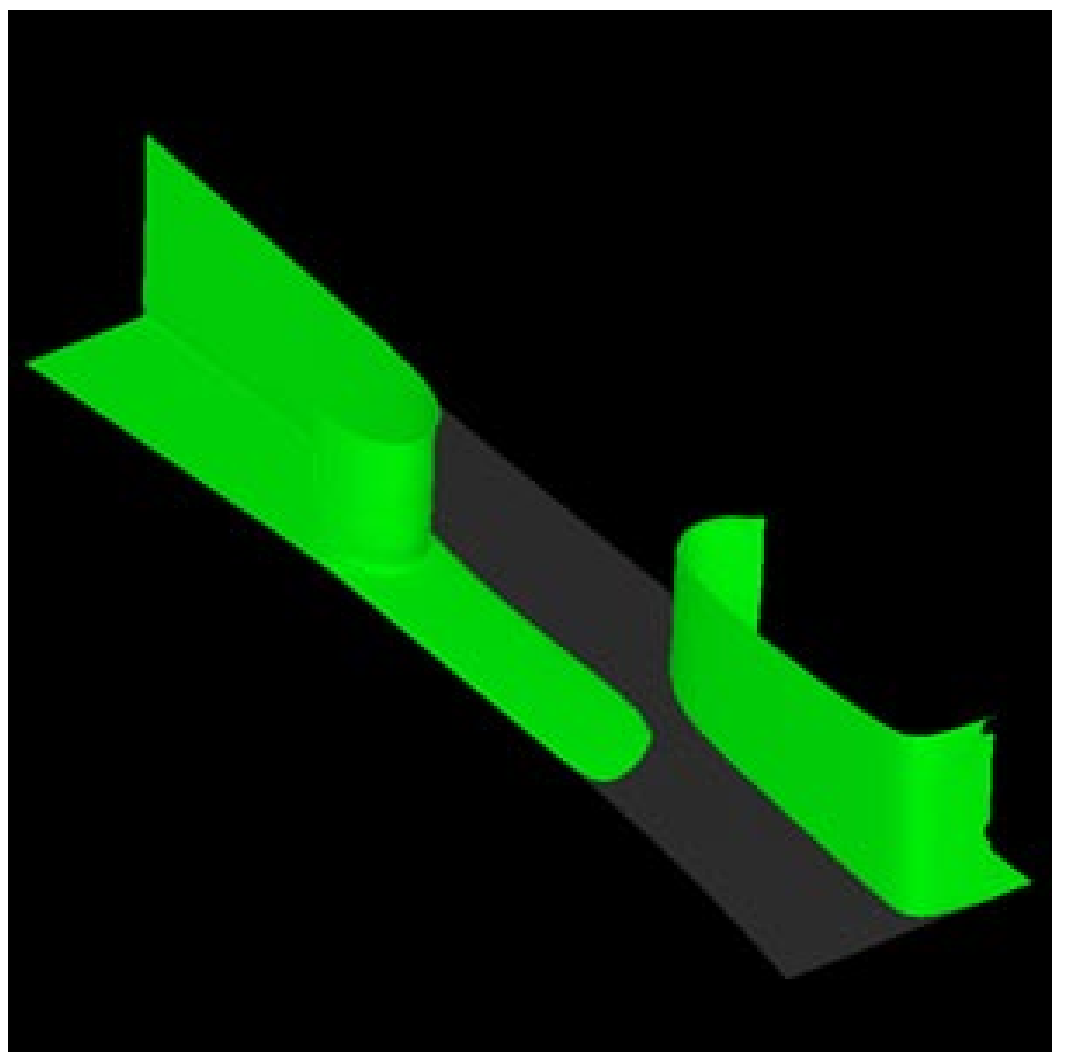

Pressure, $\mathrm{P}=97613 \mathrm{~Pa}$

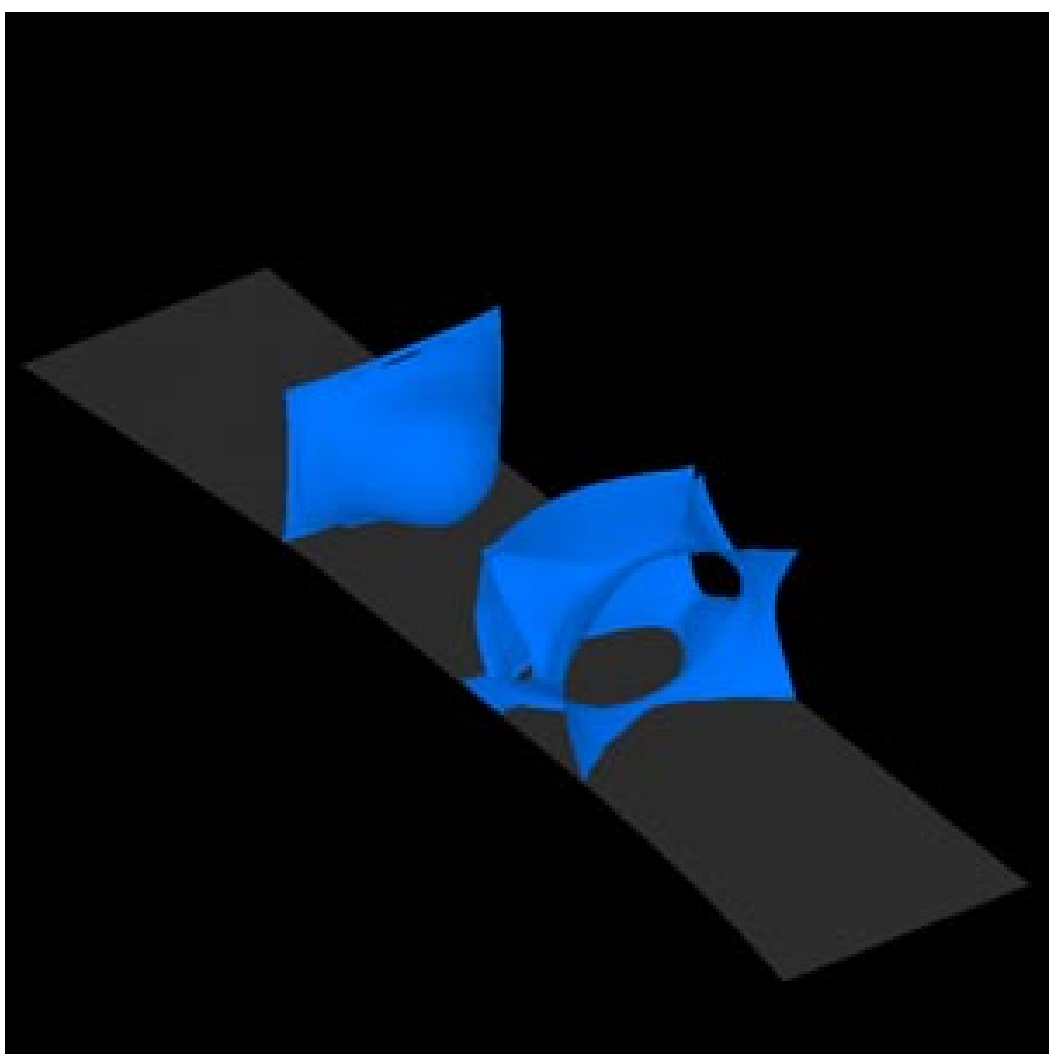




\section{Inflow Velocity Profile (Test Section)}

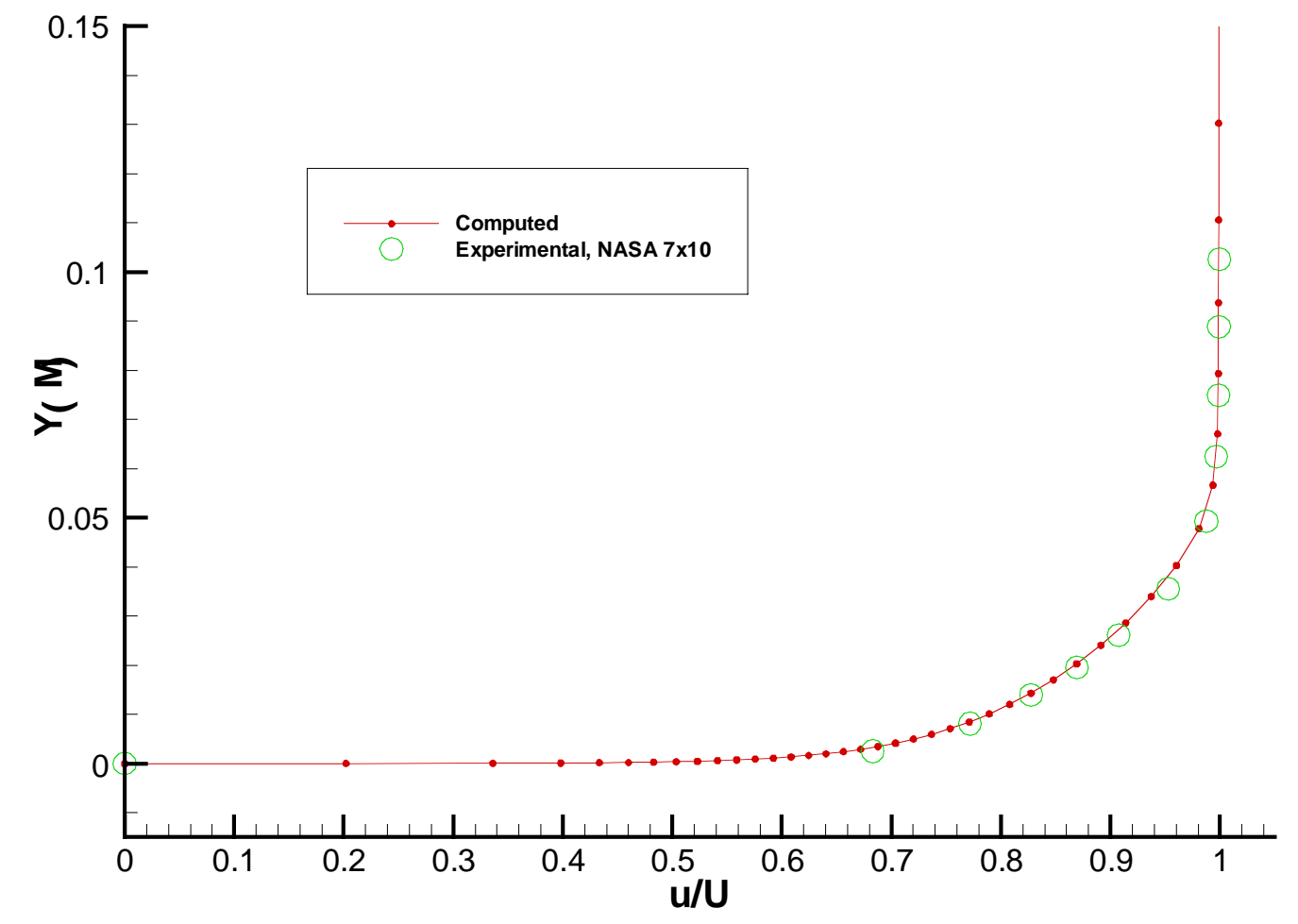




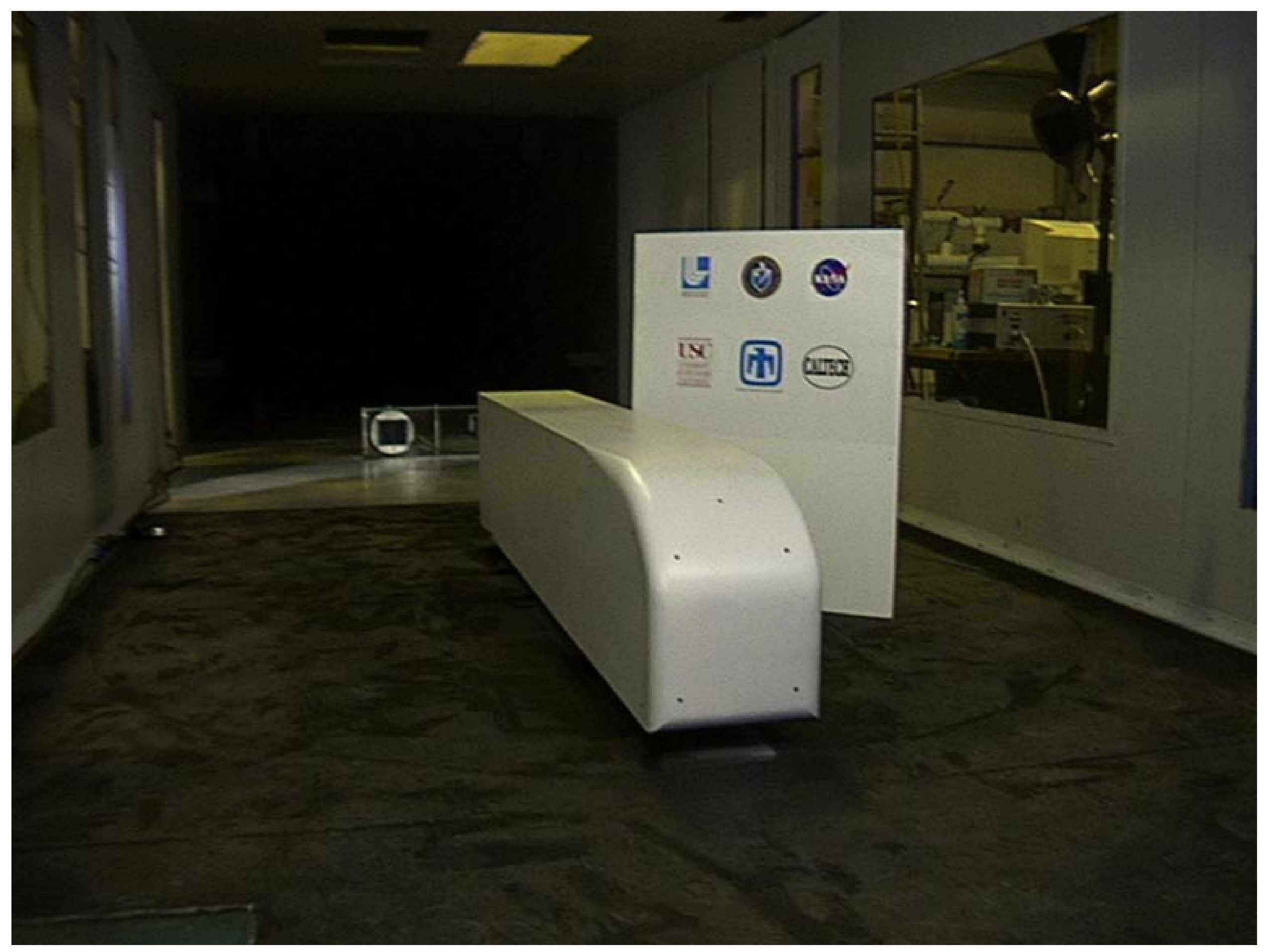




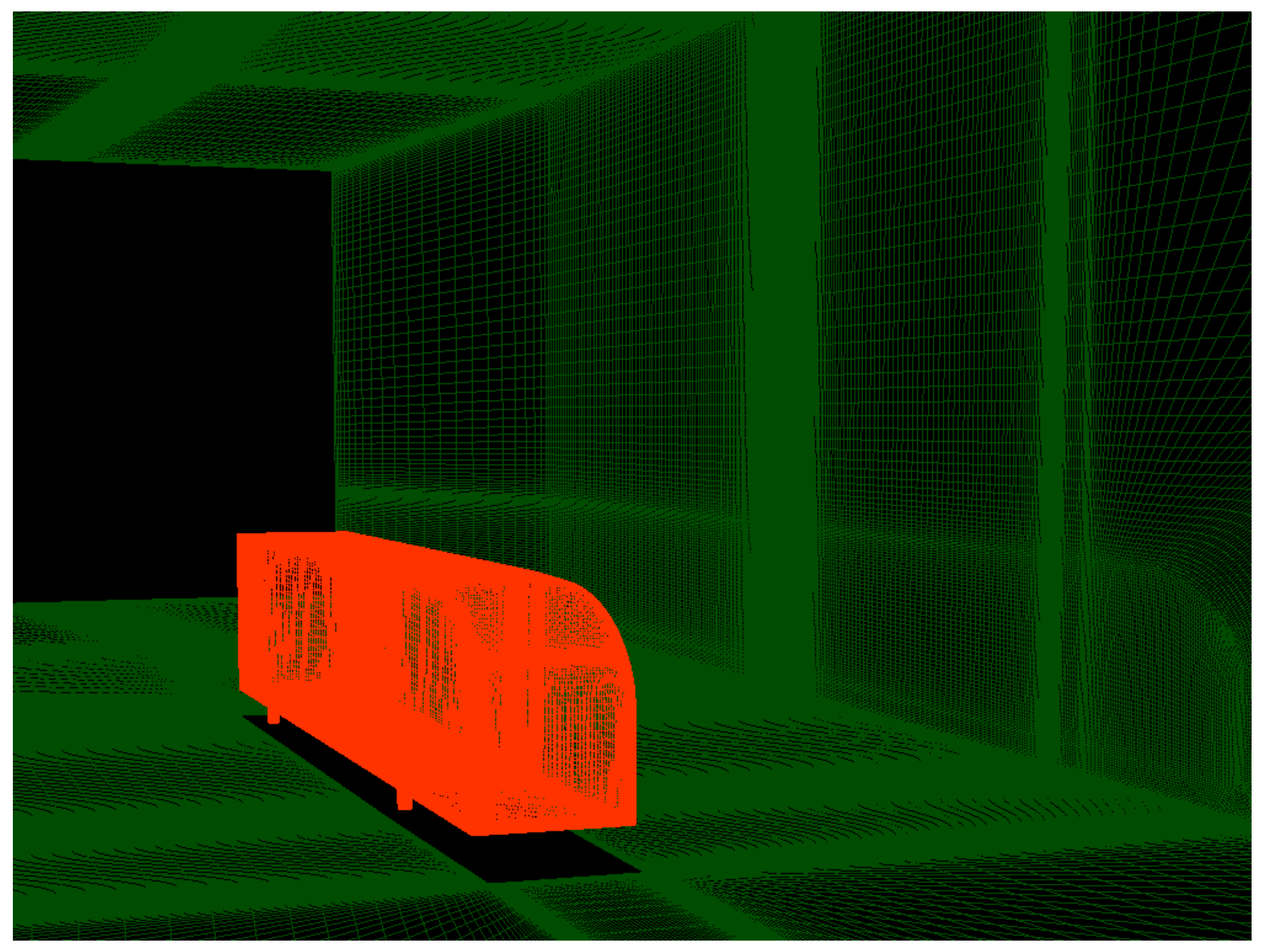




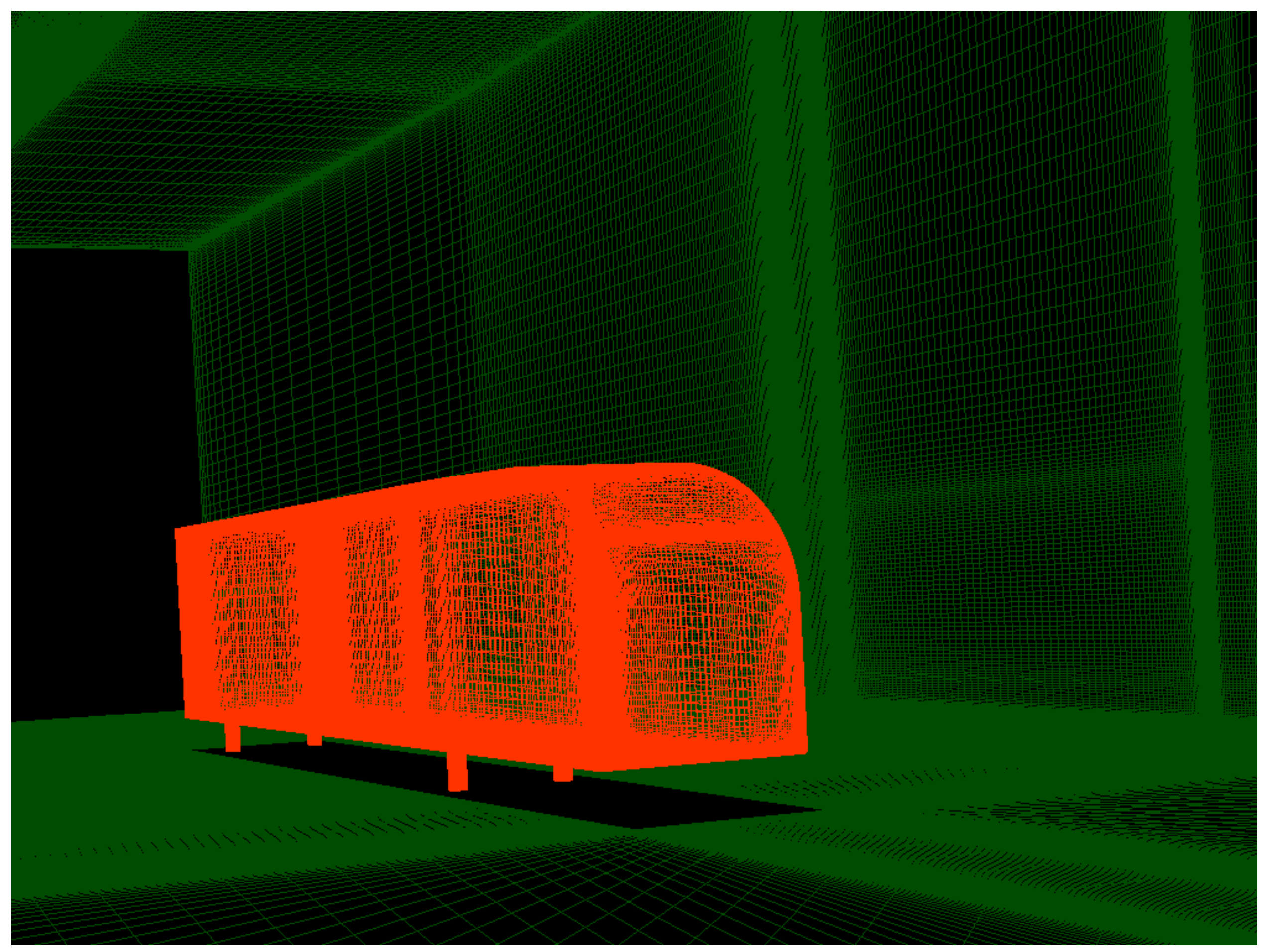




\section{Particle Traces, $0^{\circ}$ yaw}

Particle traces are colored by pressure magnitude

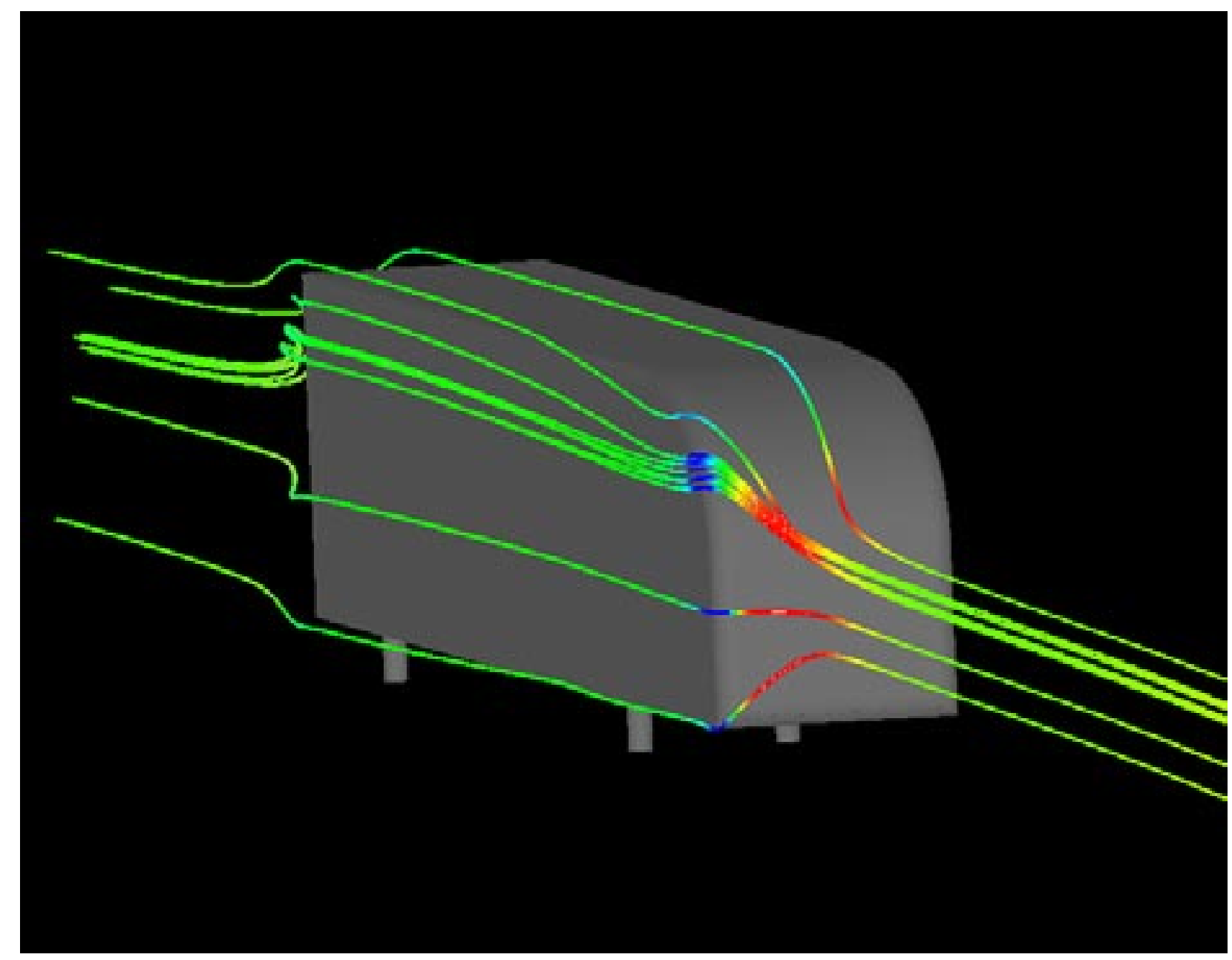




\section{Surface Pressure Coefficient $\left(C_{p}\right)$ Comparison to NASA Data}

$\mathrm{Y}=1.39$ (top of the truck), $\mathrm{Z}=0.0$ (centerline)

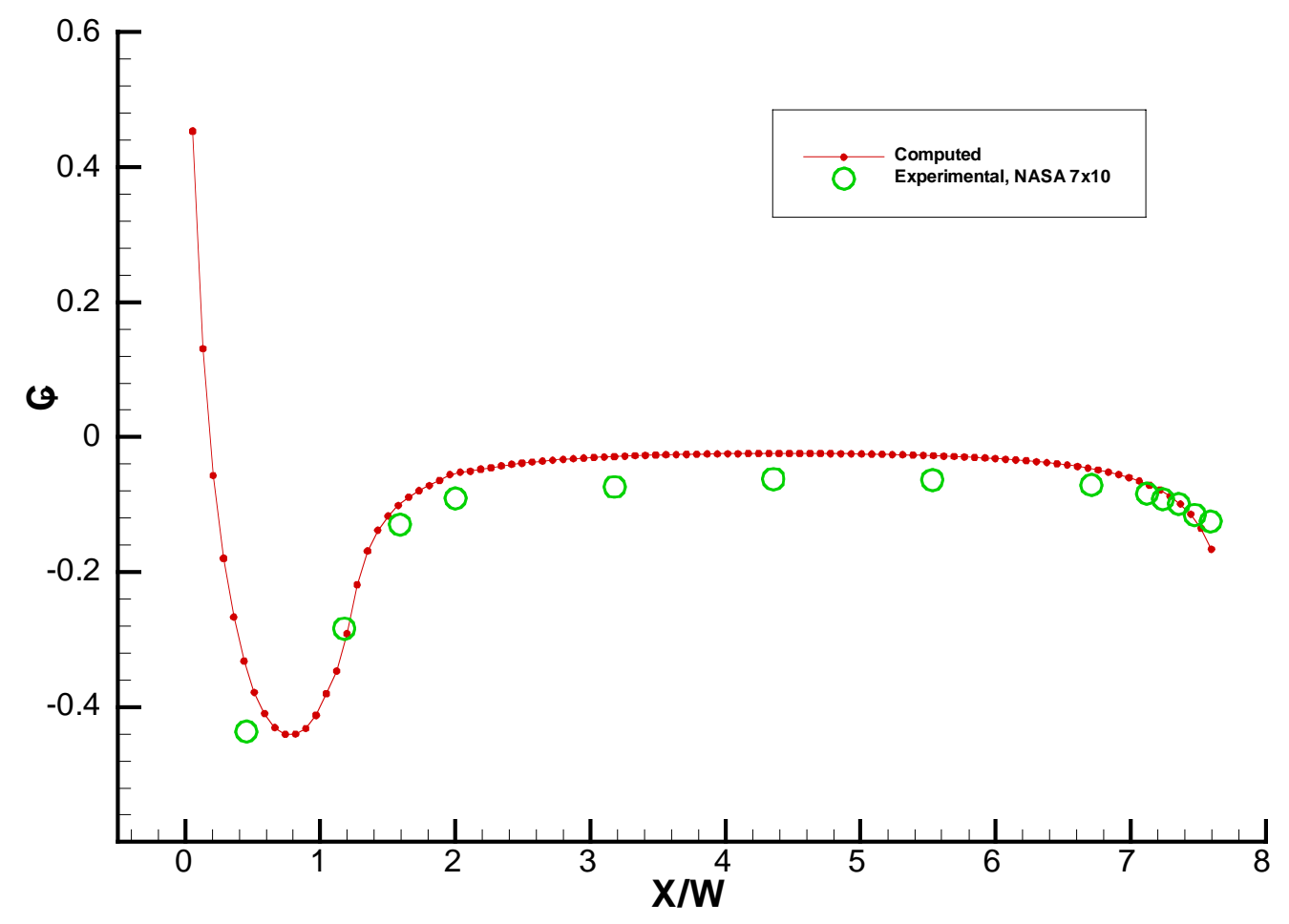




\section{Surface Pressure Coefficient $\left(C_{p}\right)$ Comparison to NASA Data}

$\mathrm{Y}=0.0$ (bottom of the truck), $\mathrm{Z}=0.0$ (centerline)

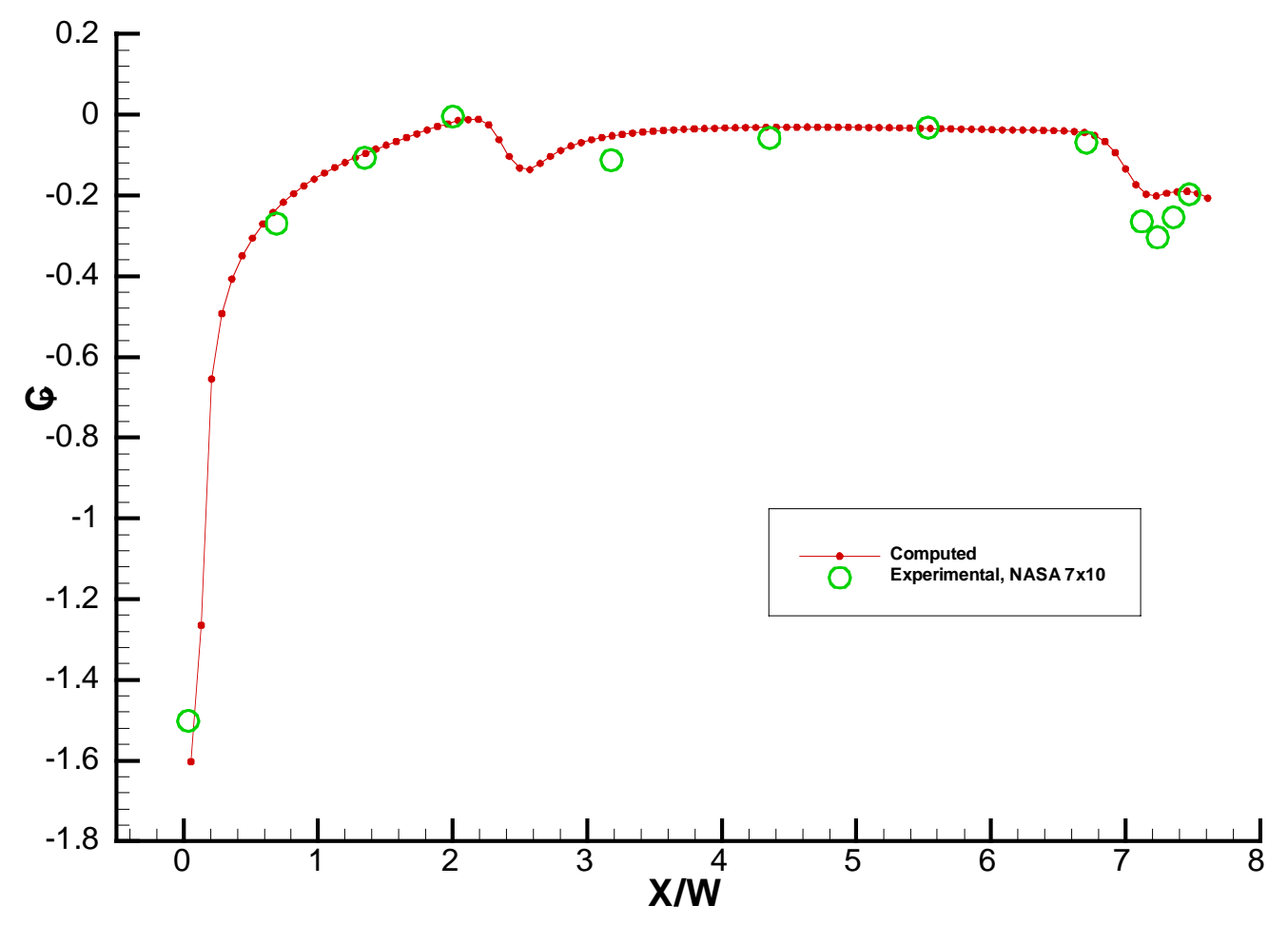




\section{Surface Pressure Coefficient $\left(C_{p}\right)$ Comparison to NASA Data}

$\mathrm{Z}=0.0$

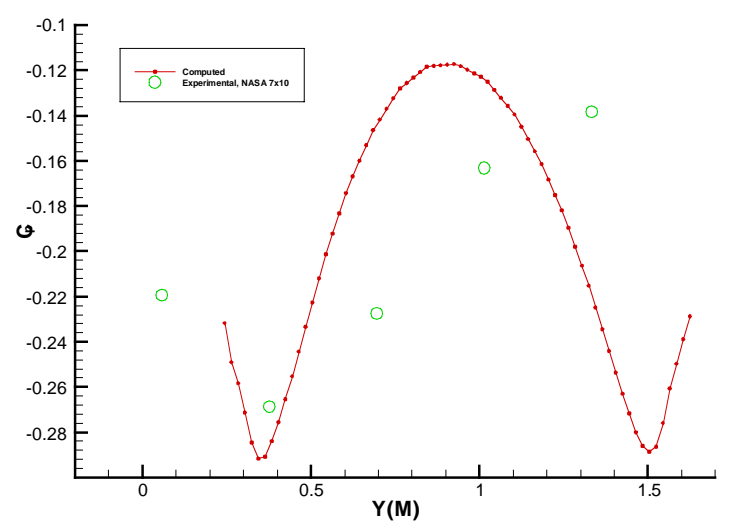

$\mathrm{Z}=0.22$

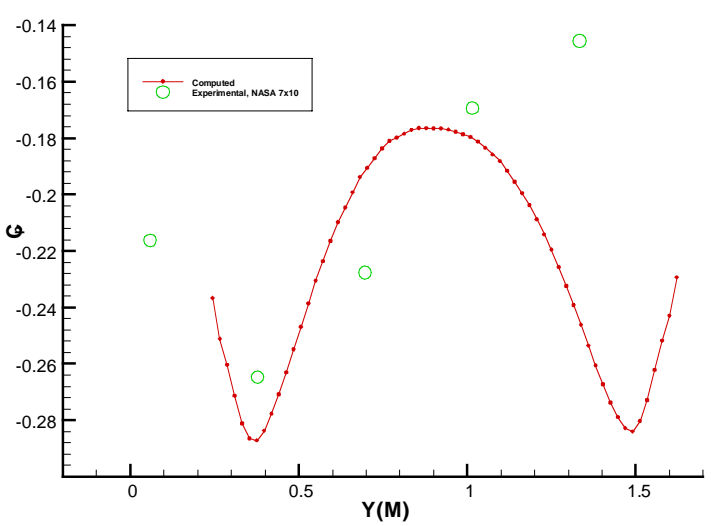

$Z=0.44$

$X=7.59$ (back of the truck) $\mathrm{Z}=0.0$ (centerline)

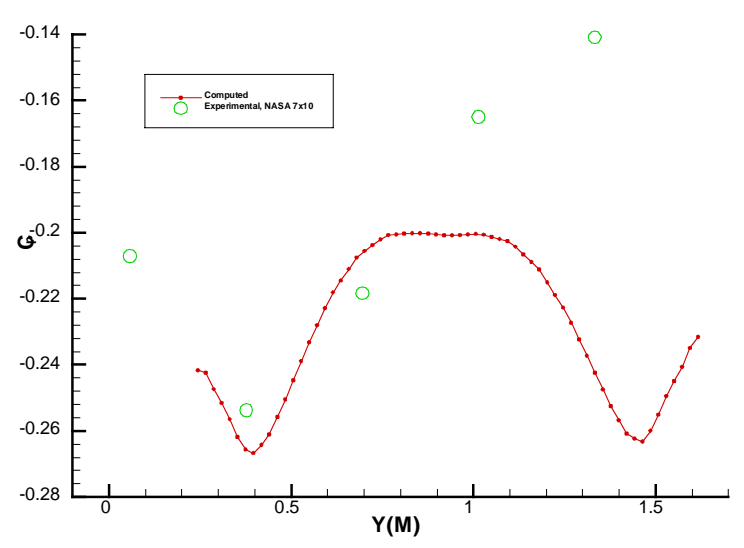




\section{Surface Pressure Coefficient $\left(C_{p}\right)$ Comparison to NASA Data}

$X=0.0$ (front of the truck), $Z=0.0$ (centerline)

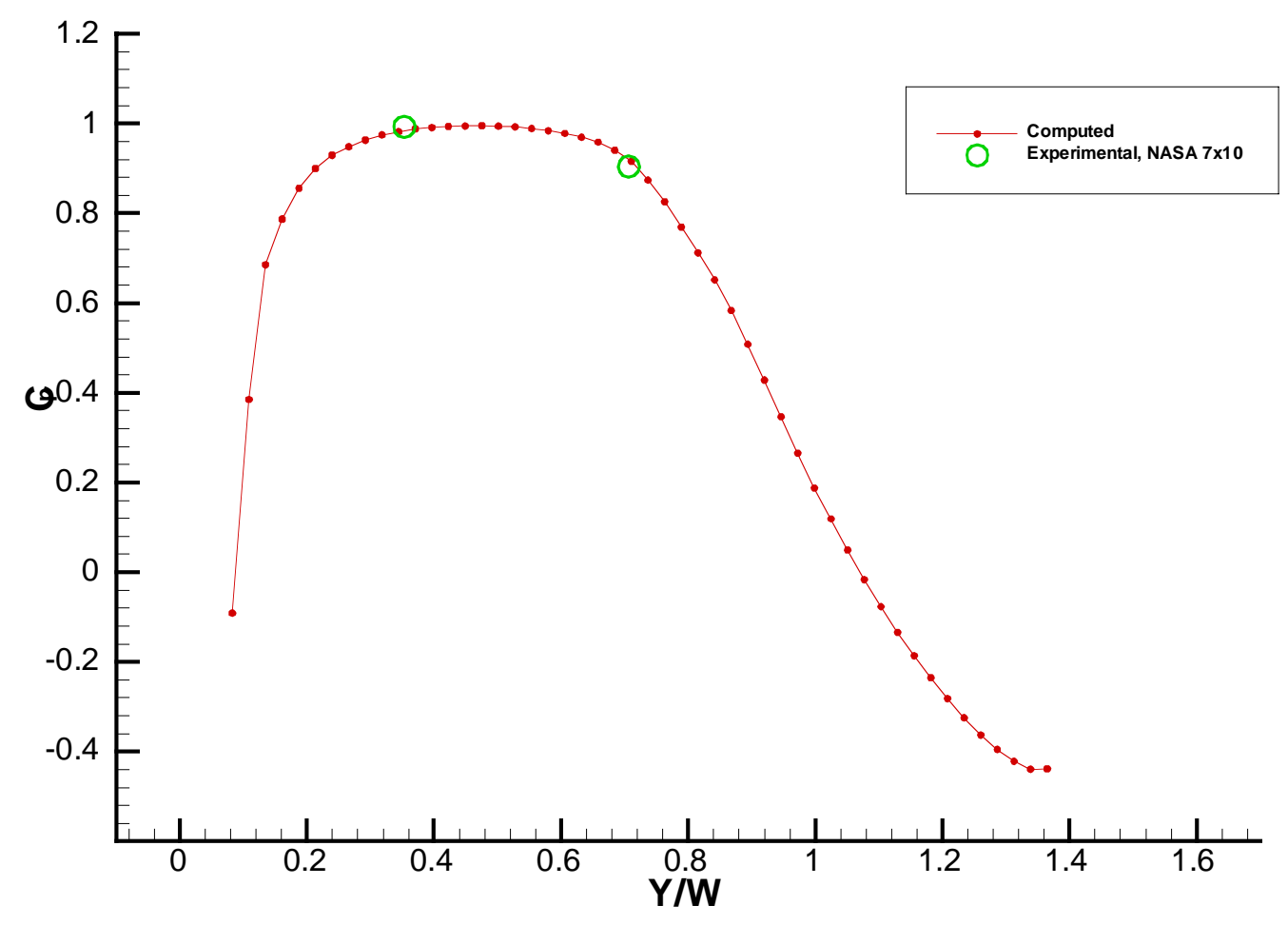




\section{Pressure Distribution on the Surface}

$0^{\circ}$ yaw

NASA
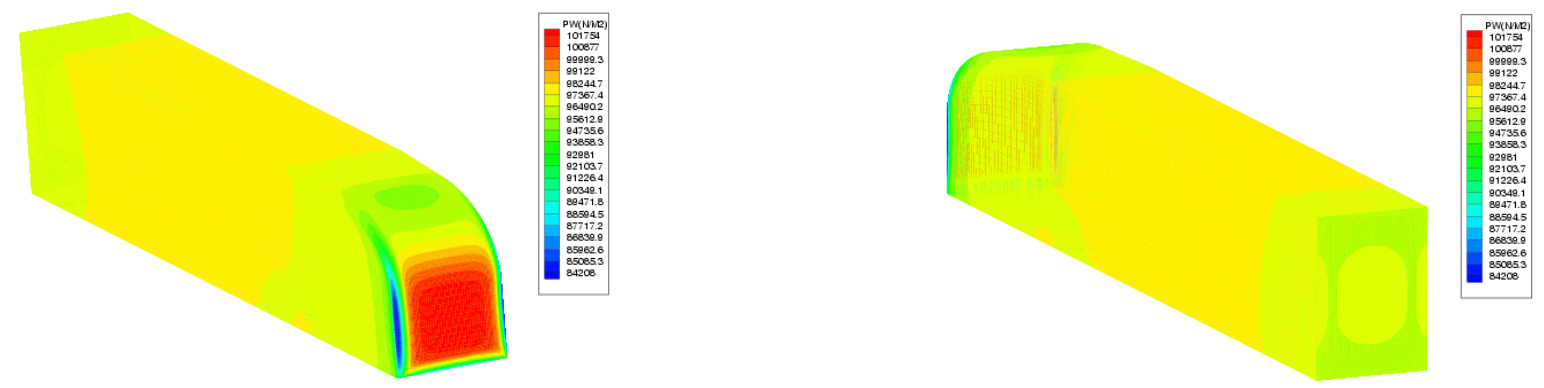

TAMU
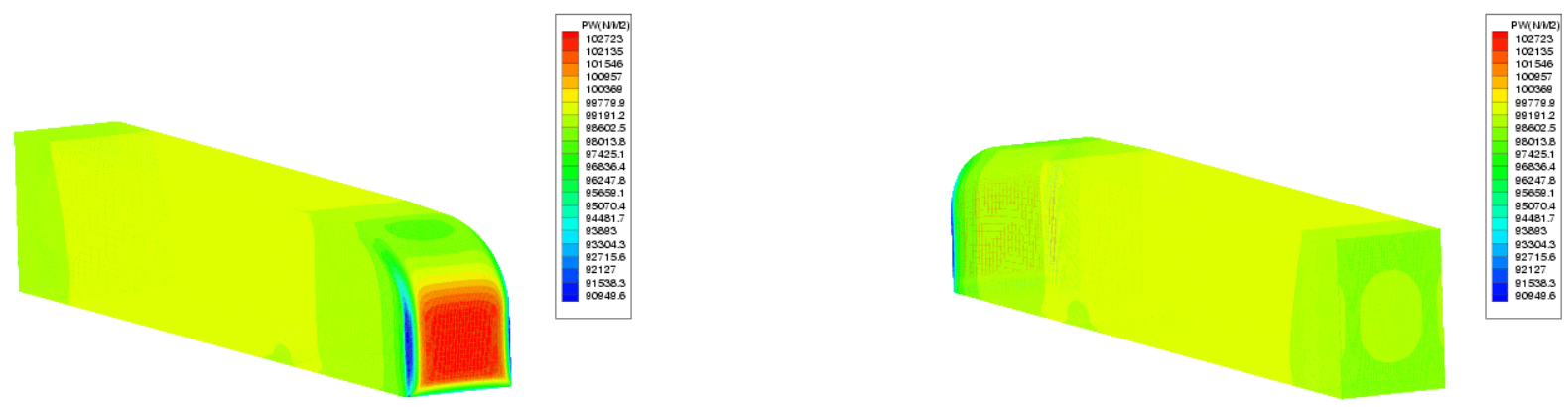


\section{Shear Stress Distribution on the Surface}

\section{$0^{\circ}$ yaw}

NASA
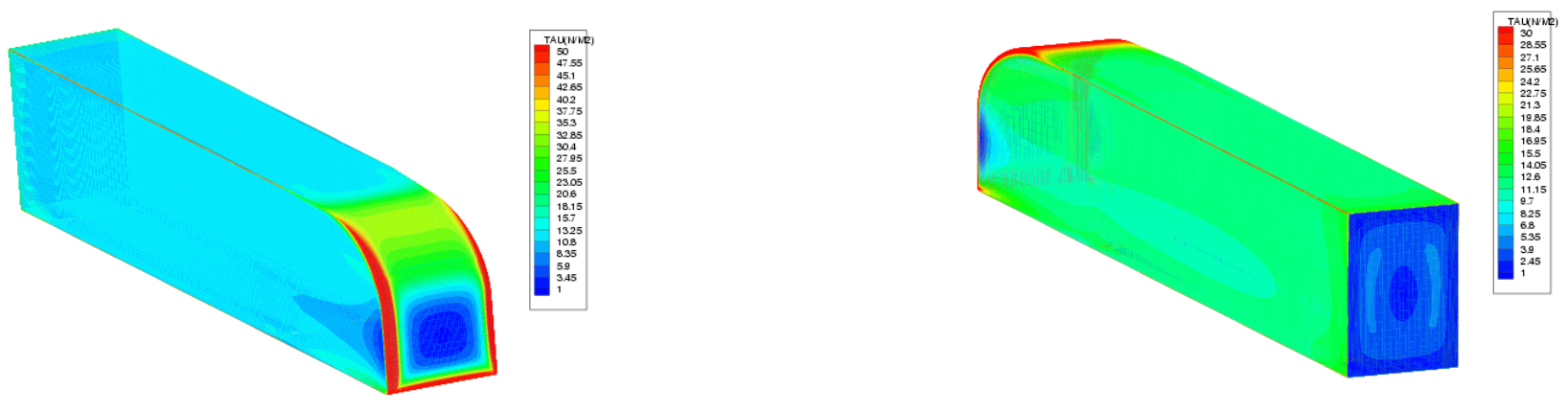

\section{TAMU}
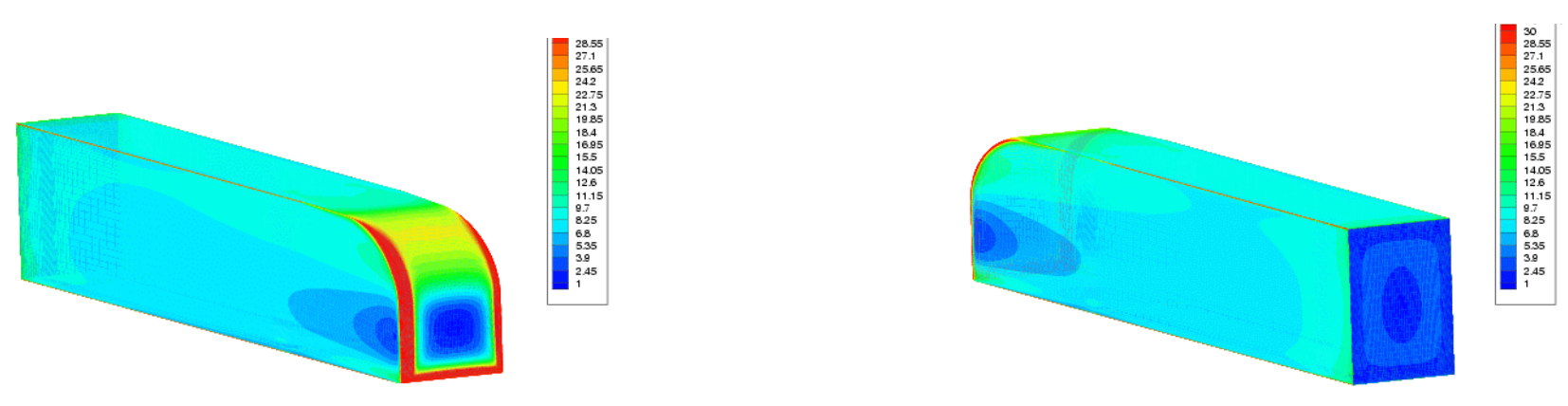


\section{Particle Traces, $10^{\circ}$ yaw}

Particle traces are colored by pressure magnitude
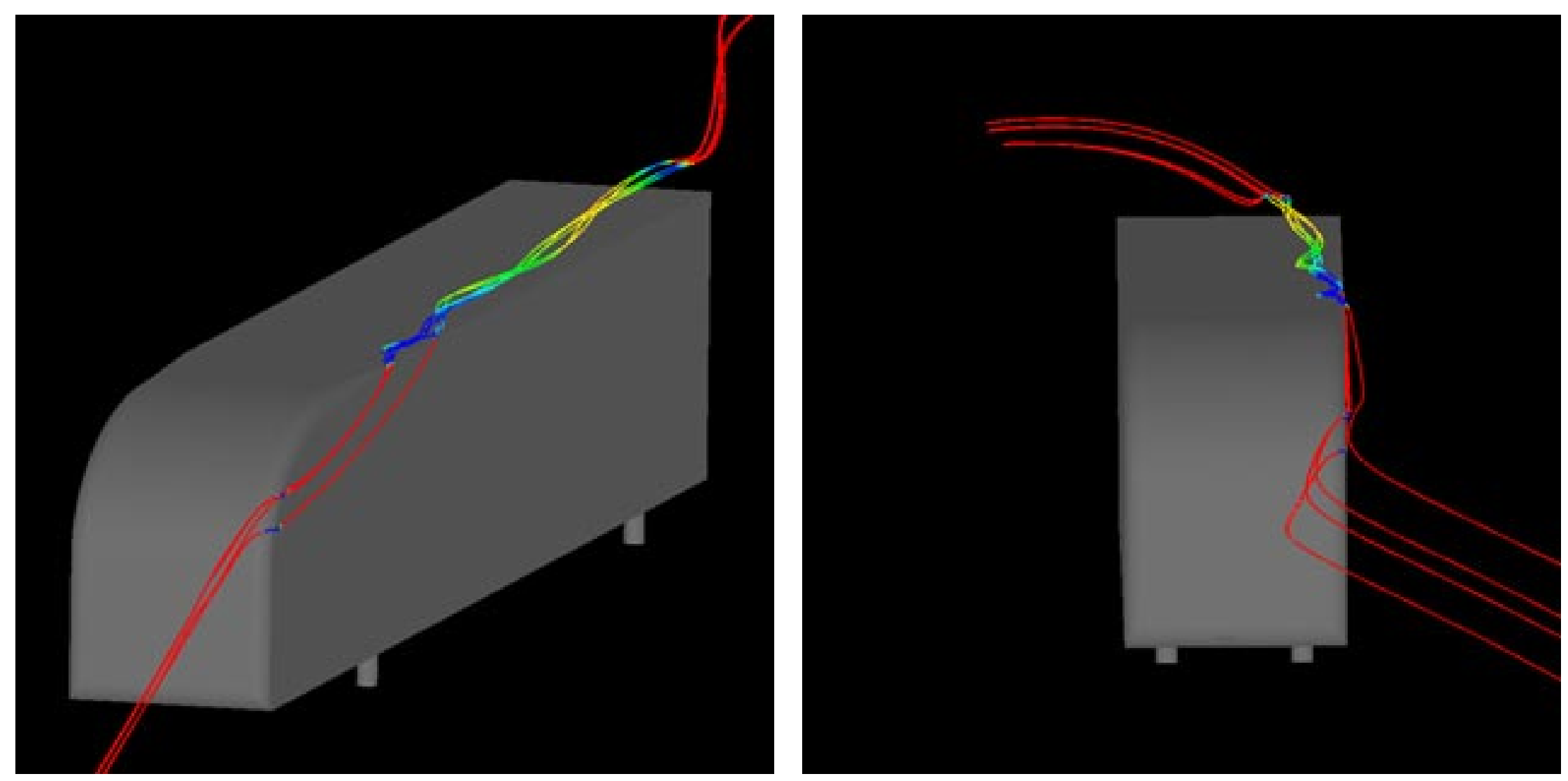


\section{Particle Traces, $10^{\circ}$ yaw}

Particle traces are colored by pressure magnitude

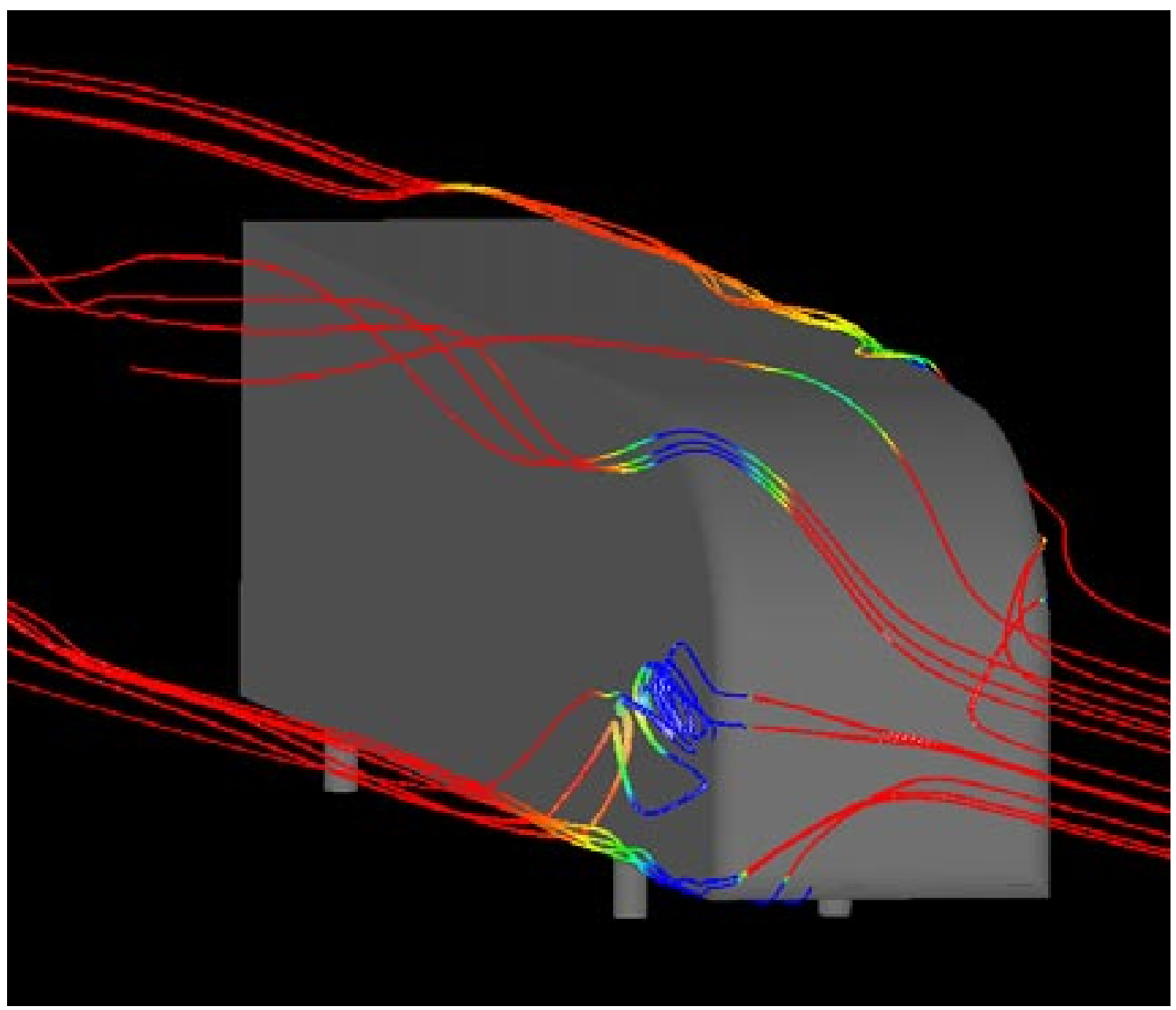




\section{Separation Bubble, Leeward Side, $10^{\circ}$ yaw}

Iso-Surface $\mathbf{u}=\mathbf{- 0 . 0 0 1}(\mathrm{m} / \mathrm{s})$

NASA

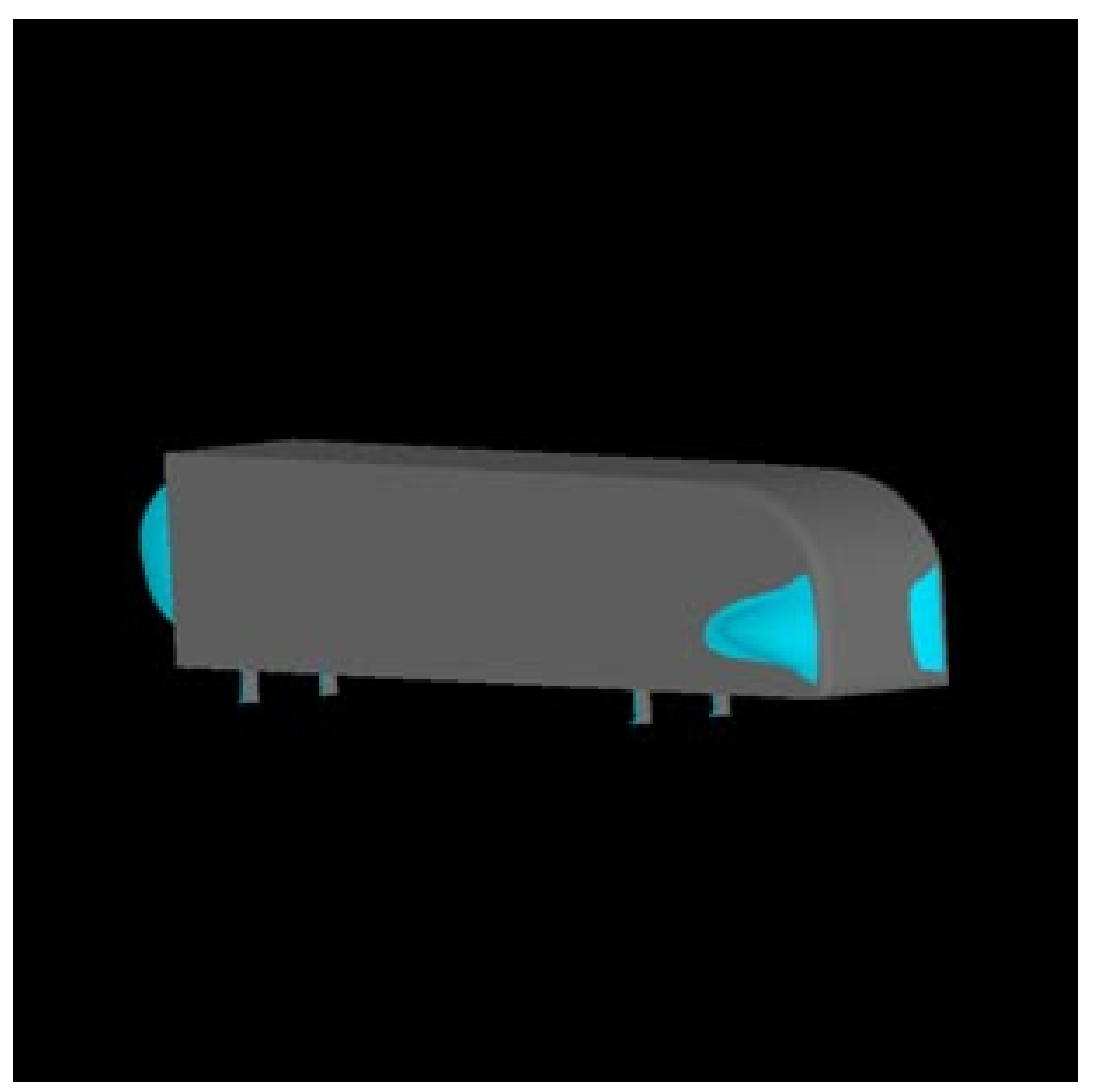

TAMU

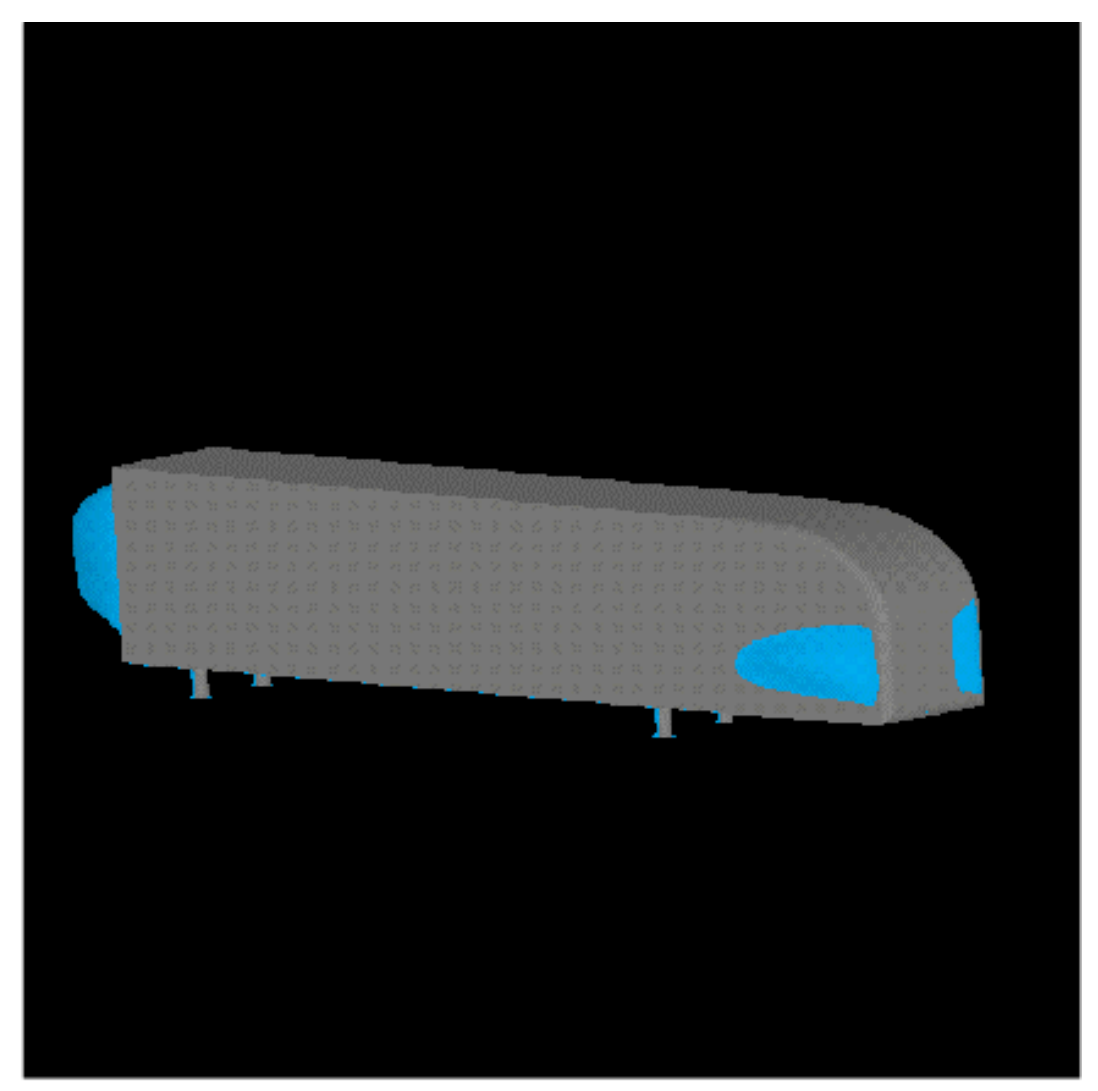




\section{Separation Bubble, Leeward Side, $10^{\circ}$ yaw, ...}

Iso-Surface $\mathbf{u}=\mathbf{- 0 . 0 0 1}(\mathrm{m} / \mathrm{s})$
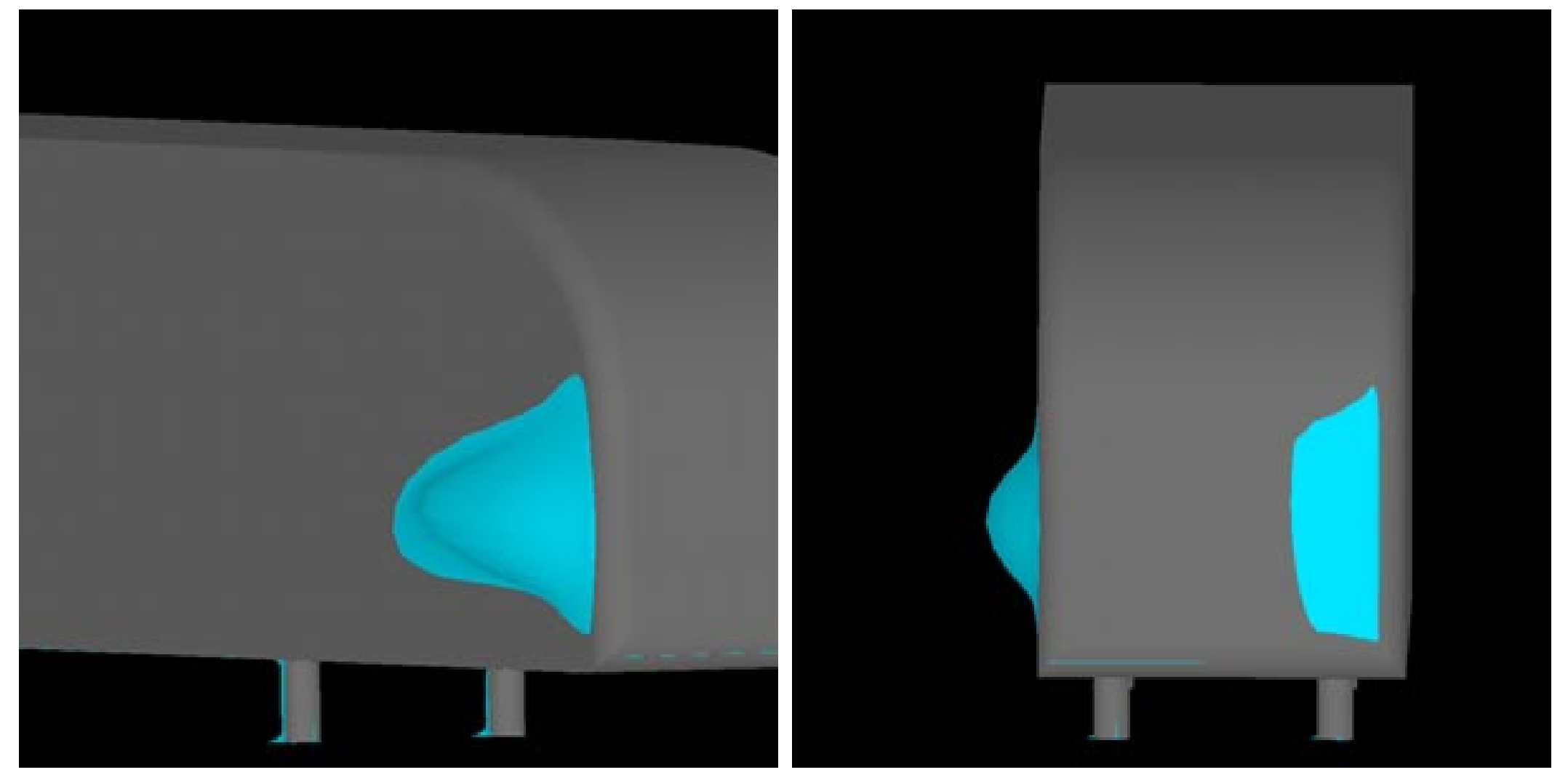


\section{Pressure Distribution on the Surface}

$$
10^{\circ} \text { yaw }
$$

NASA
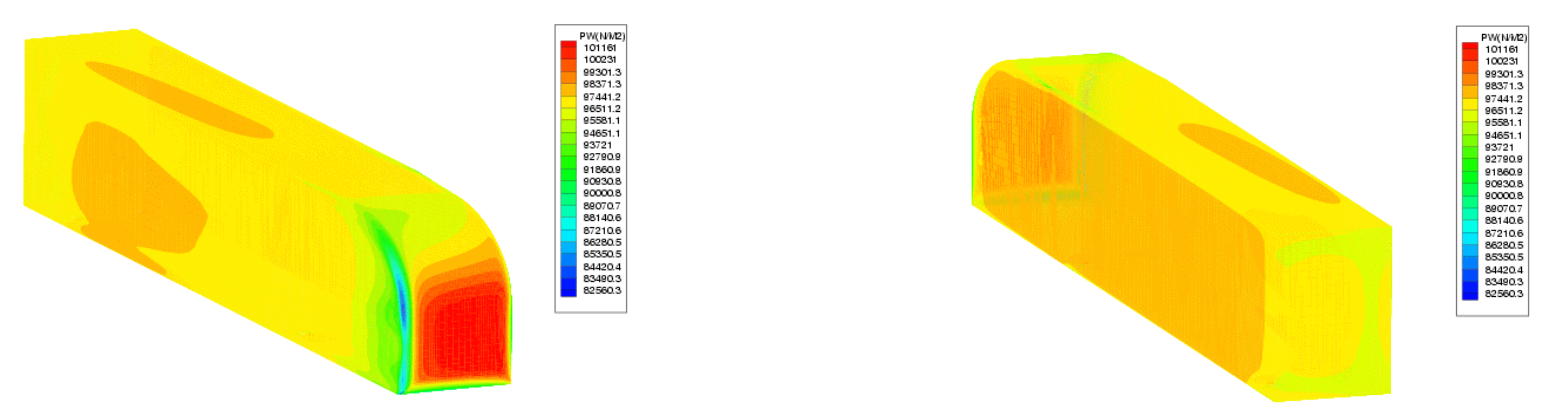

TAMU
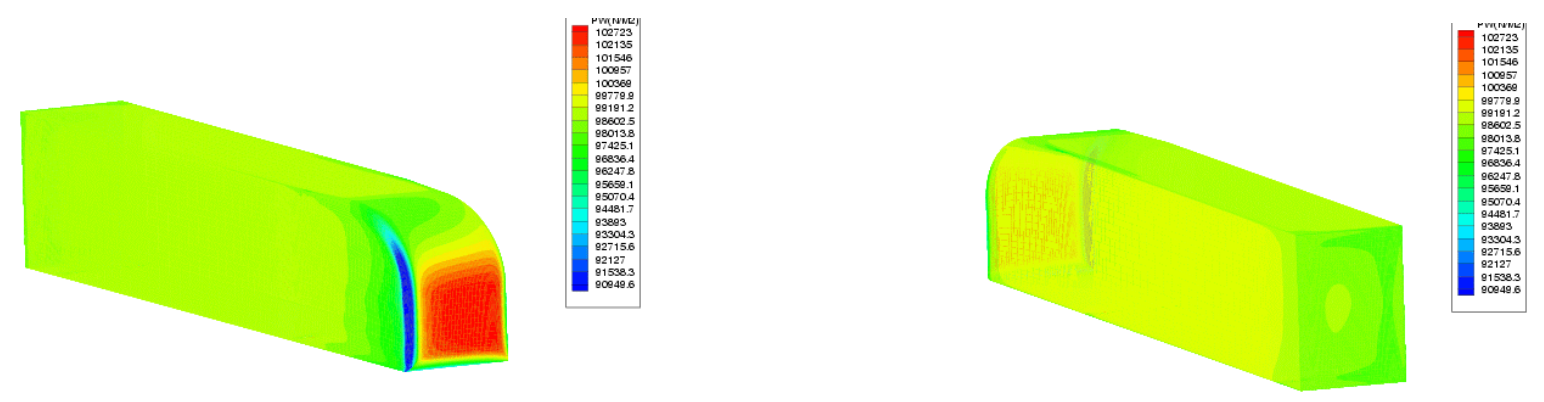


\section{Pressure Sensitive Paint (NASA)}

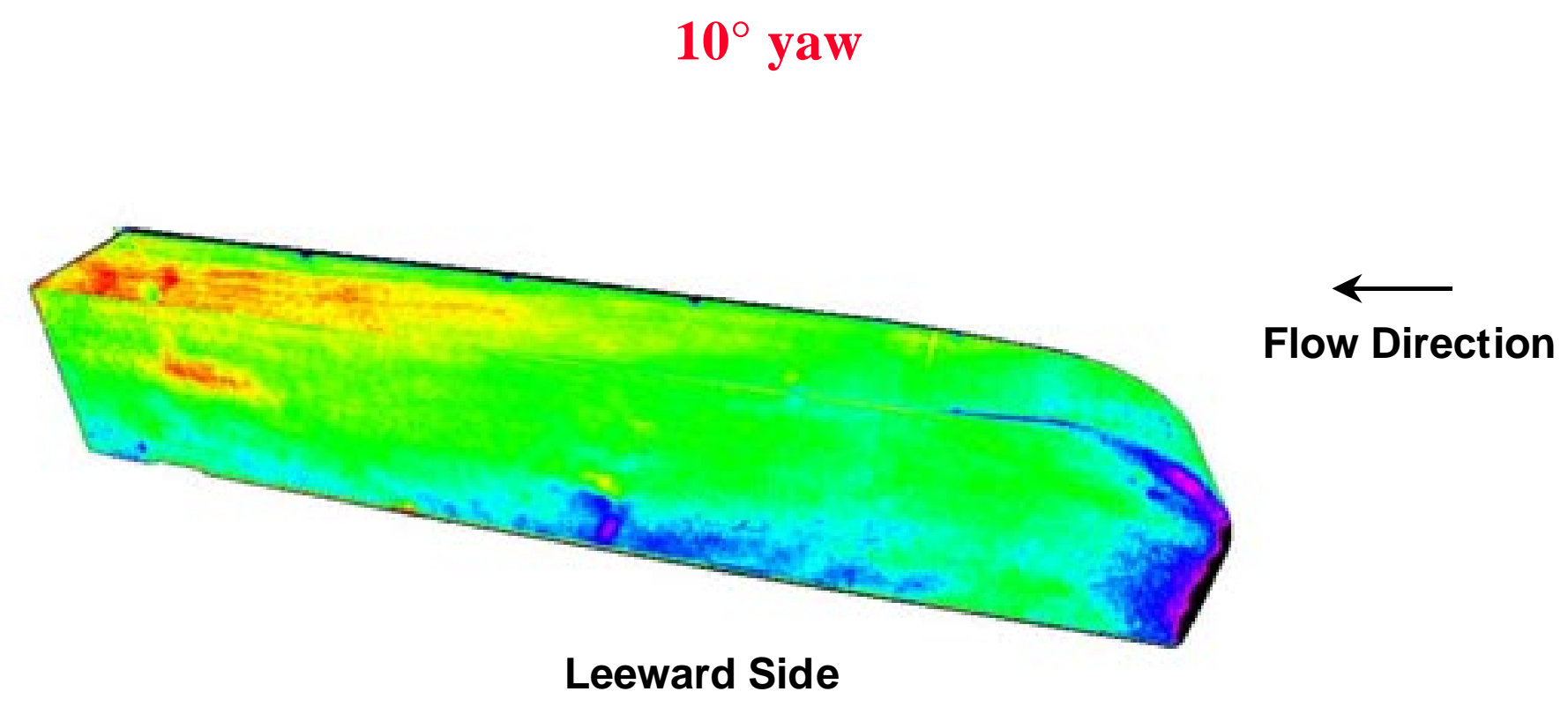




\section{Shear Stress Distribution on the Surface}

$$
10^{\circ} \text { yaw }
$$

NASA
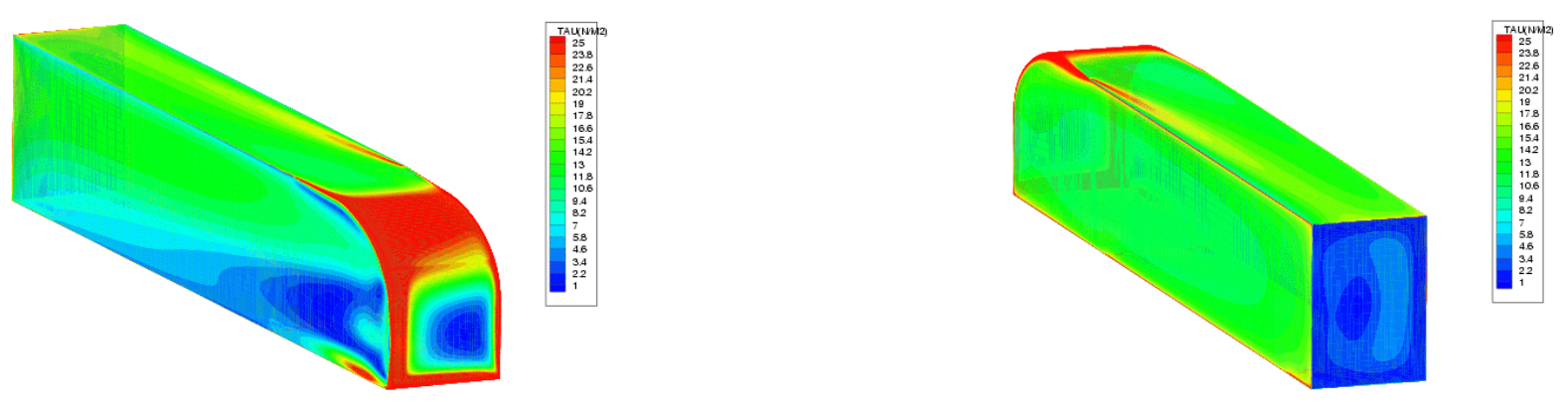

TAMU
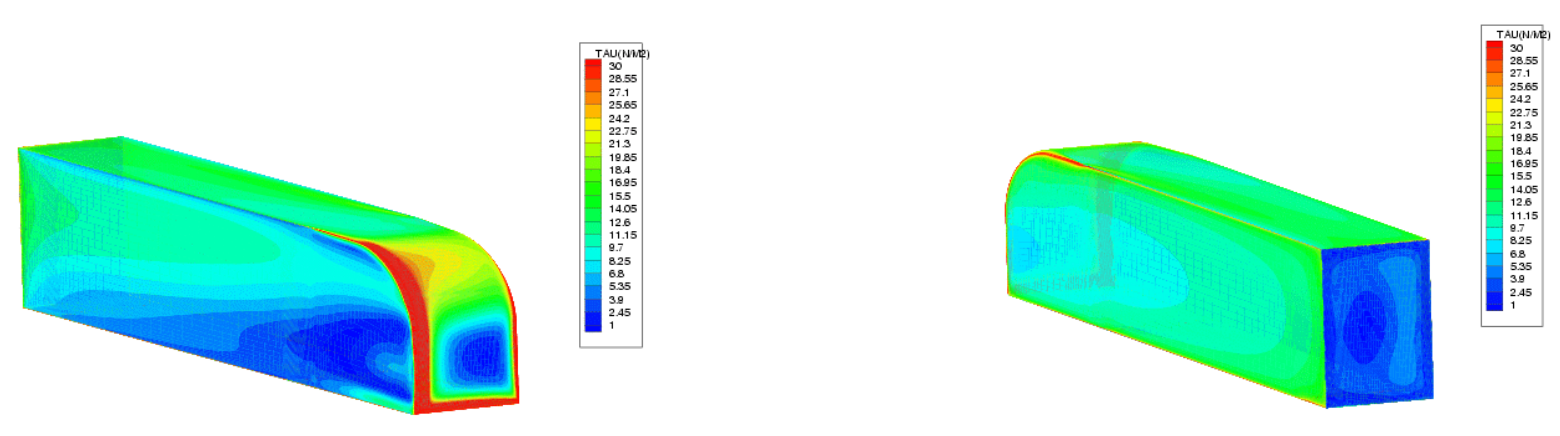


\section{Concluding Remarks}

- It was important (for validation purposes) to perform "tunnel empty" simulation of the NASA 7x10 tunnel to obtain proper boundary conditions for GTS simulation

- Two yaw angles ( $0^{\circ}$ and $\left.10^{\circ}\right)$ were computed and the result for the 0 yaw was compared to NASA data

- There is a question of how $C_{p}$ should be computed to be consistent with the experimental calculation (current method introduces data reduction uncertainty) 


\section{Sandia Near Term Tasks}

- Complete 0 \& 10 yaw simulations and compare results to NASA $7^{\prime} \times 10^{\prime}$ experimental data

- Initiate Boattail Plate calculations

- Perform Grid Convergence Studies on 10 yaw case

- Initiate simulations for additional yaw angles to obtain "Wind Averaged Drag Coefficient"

- Document "effort to date" with NASA configuration in technical report (FY01) 
TruCK AERODYNAMICS:

\title{
Progress in Code Development Related to TruCK AEROdynamics
}

\author{
Tim Dunn Rose McCallen Dan Flowers \\ Lawrence Livermore National Laboratory
}

March 16, 2000 


\section{OUR GOAL IS NUMERICAL SOLUTIONS OF THE COMPRESSIBLE AND INCOMPRESSIBLE N-S EQUATIONS.}

\section{Status:}

Compressible

Incompressible
In production mode.

Enhancements continually being made.

Currently in development.

Working for small problems.

Working up to large problems.

\section{Common Themes:}

Finite Element Method (FEM)

Unstructured Grid

Large-Eddy Simulation

Time-Accurate

- Explicit Time-Stepping 


\section{Compressible Flow}

- Large Mach numbers OK

- Time-step limited by speed of sound

$$
\left[\Delta t \sim \frac{1}{a}\right]
$$

\section{Incompressible Flow}

- Small Mach numbers only $(\mathrm{M}<0.1-0.3)$

- Time-step limited by local flow velocity

$$
\left[\Delta t \sim \frac{1}{u}\right]
$$




\section{THE COMPRESSIBLE FLOW USES AN}

\section{ARbitrary LAgRANGIAN EULERIAN ForMULATION.}

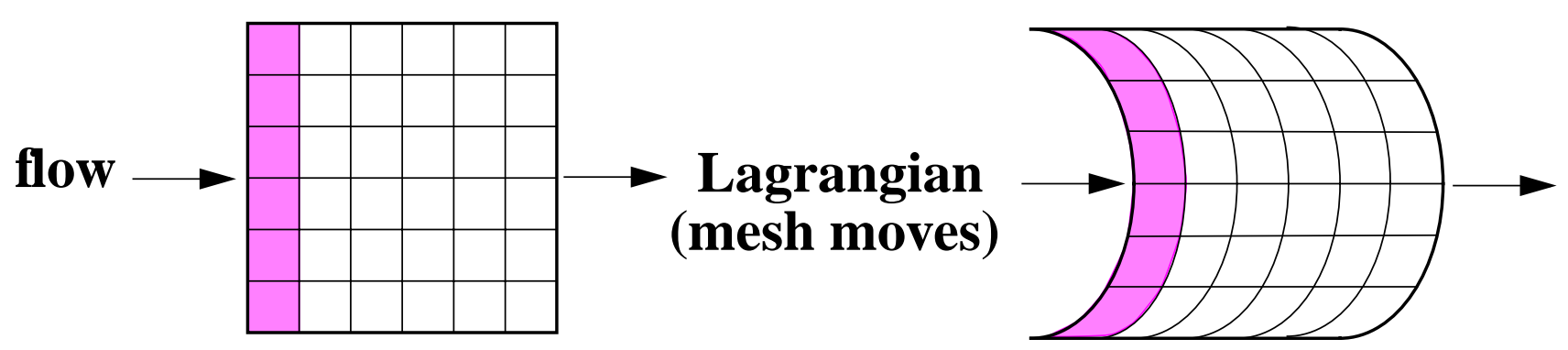

accurate but can tangle

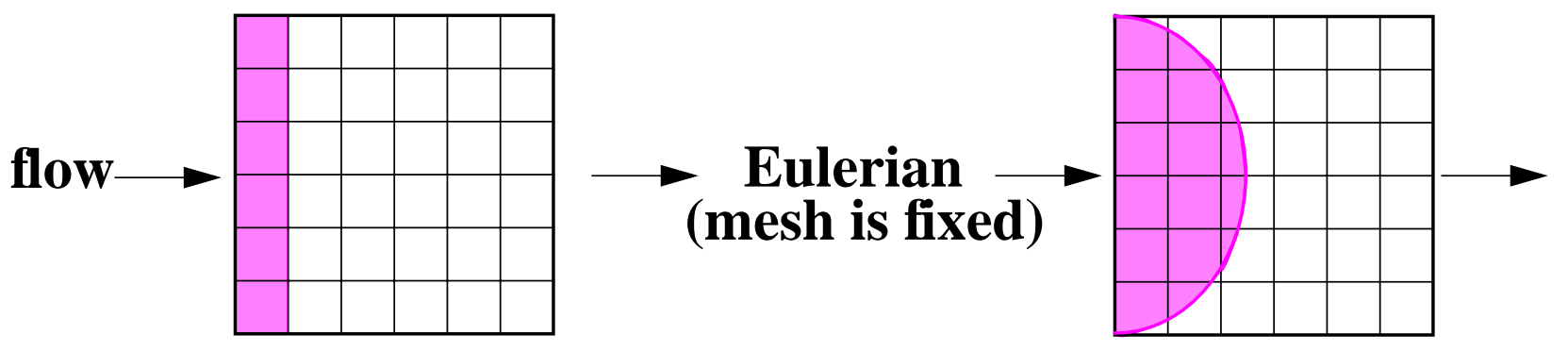

will not tangle, but may need

ALE fine resolution everywhere

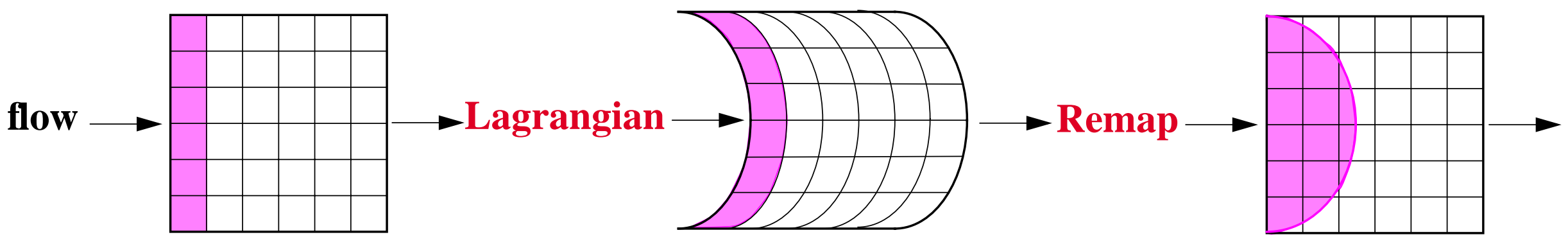




\section{THE INCOMPRESSIBLE FLOW USES AN}

EULERIAN FORMULATION.

Galerkin Finite-Element Method

- 8-node Hexahedral Elements

- Tri-linear Basis Functions for Velocity

- Piecewise Constant Pressure

- Single Point Integration 


\section{THE INCOMPRESSIBLE FLOW EQUATIONS}

\section{Conservative Form}

Mass

$$
\frac{\partial u_{\alpha}}{\partial x_{\alpha}}=0
$$$$
\tau_{\alpha \beta}=-P \delta_{\alpha \beta}+v \frac{\partial u_{\alpha}}{\partial x_{\beta}}
$$

Momentum

$$
\frac{\partial u_{\alpha}}{\partial t}+u_{\beta} \frac{\partial u_{\alpha}}{\partial x_{\beta}}=\frac{\partial \tau_{\alpha \beta}}{\partial x_{\beta}}
$$$$
P=\frac{p}{\rho}
$$

Variational Form

Mass

$$
\int_{\Omega} w \frac{\partial u_{\alpha}}{\partial x_{\alpha}}=0
$$

Momentum

$$
\begin{aligned}
& \int_{\Omega} v\left(\frac{\partial u_{\alpha}}{\partial t}+u_{\beta} \frac{\partial u_{\alpha}}{\partial x_{\beta}}\right)+\int_{\Omega} \tau_{\alpha \beta} \frac{\partial v}{\partial x_{\beta}}=\int_{\partial \Omega} v f_{\alpha} \\
& f_{\alpha}=n_{\beta} \tau_{\alpha \beta}=n_{\beta}\left(-P \delta_{\alpha \beta}+v \frac{\partial u_{\alpha}}{\partial x_{\beta}}\right)
\end{aligned}
$$




\section{THE INCOMPRESSIBLE FLOW EQUATIONS DISCRETE FORM}

Variable Expansion

$$
\begin{aligned}
u_{\alpha}^{h}(x, t)=\sum_{j=1}^{N} u_{\alpha}^{j}(t) \phi_{j}(x) & v=\phi_{i}(x), i=1, N \\
h_{(x, t)}=\sum_{j=1}^{M} P_{j}(t) \psi_{j}(x) & w=\psi_{i}(x), i=1, M
\end{aligned}
$$

Mass

$$
\left(\int_{\Omega} \psi_{i} \frac{\partial \phi_{j}}{\partial x_{\alpha}}\right) u_{\alpha}^{j}=0
$$

Momentum

$$
\begin{array}{r}
\left(\int_{\Omega} \phi_{i} \phi_{j}\right) \frac{\partial u_{\alpha}^{j}}{\partial t}+\left(u_{\beta}^{k} \int_{\Omega} \phi_{i} \phi_{k} \frac{\partial \phi_{j}}{\partial x_{\beta}}\right) u_{\alpha}^{j}+\left(\int_{\Omega} v \frac{\partial \phi_{i}}{\partial x_{\beta}} \frac{\partial \phi_{j}}{\partial x_{\beta}}\right) u_{\alpha}^{j} \\
-\left(\int_{\Omega} \psi_{j} \frac{\partial \phi_{i}}{\partial x_{\alpha}}\right) P^{j}=\int_{\partial \Omega} \phi_{i} f_{\alpha}
\end{array}
$$




\section{THE INCOMPRESSIBLE FLOW EQUATIONS MATRIX FORM}

$$
\begin{gathered}
C^{T} \underline{v}=0 \\
M \dot{u}+[K+N(u)] u+C P=F
\end{gathered}
$$

OR

$$
\left[\begin{array}{ll}
M^{\prime} & C \\
C^{T} & 0
\end{array}\right]\left[\begin{array}{l}
v \\
P
\end{array}\right]=\left[\begin{array}{l}
F^{\prime} \\
0
\end{array}\right]
$$

where

$$
\begin{aligned}
& \underline{v}=\underline{u}^{n+1}-\underline{u}^{n} \quad M^{\prime}=\frac{M}{\Delta t} \quad F^{\prime}=F-[K+N(u)] u \\
& C=\left[\begin{array}{l}
c_{i n(1)} \\
c_{i n(2)} \\
c_{i n(3)}
\end{array}\right] ; c_{i n(\alpha)}=\int_{\Omega} \psi_{n} \frac{\partial \phi_{i}}{\partial x_{\alpha}} \quad M=\left[\begin{array}{ccc}
m_{i j} & 0 & 0 \\
0 & m_{i j} & 0 \\
0 & 0 & m_{i j}
\end{array}\right] ; m_{i j}=\int_{\Omega} \phi_{i} \phi_{j}
\end{aligned}
$$




\section{THE MATRIX IS ASSEMBLED AND SOLVED USING THE FEI DEVELOPED AT SANDia NATIONAL LAB.}

Matrix Assembly

- FEI (SNL)

Solvers

- ISIS (SNL)

- HYPRE (LLNL)

- AZTEC (SNL)

Matrix Solved

- Full Matrix

- Schur Compliment, $\left(C^{T} M^{-1} C\right) P=0$

- Difficult to find solvers which efficiently solve our matrix and work in parallel. 
WE HAVE SOME VALIDATION OF

THE INCOMPRESSIBLE FLOW MODEL.

Couette
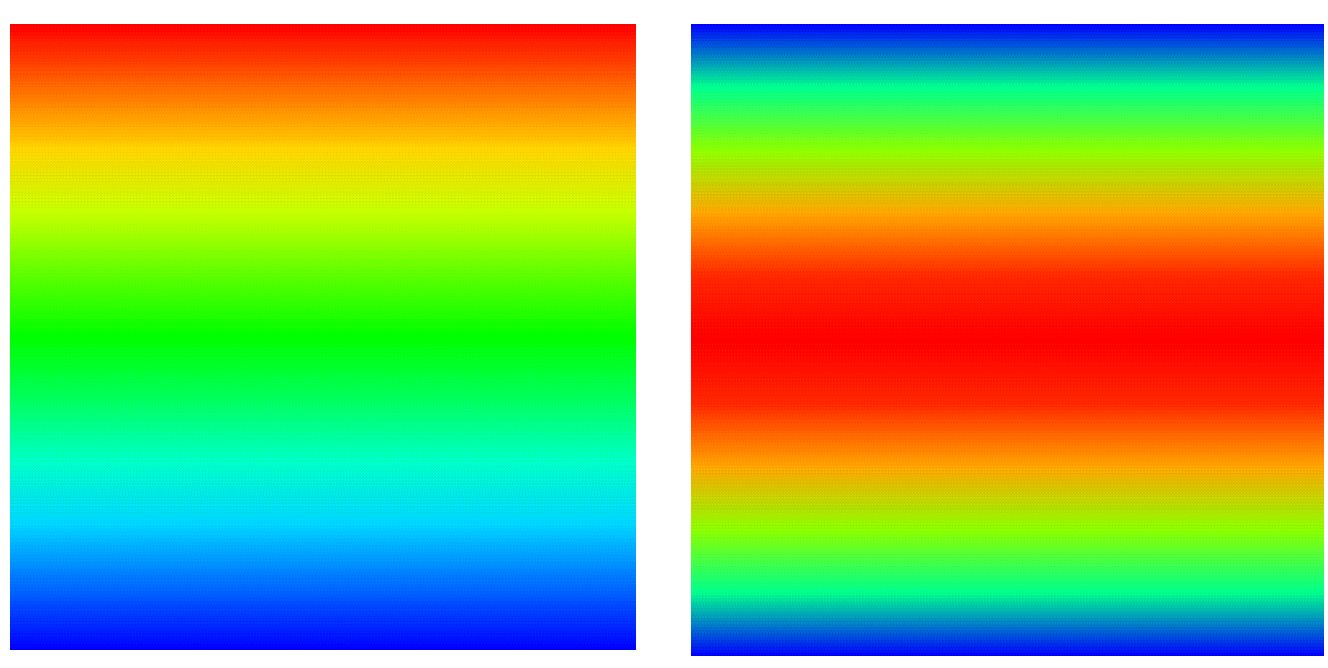

Venturi

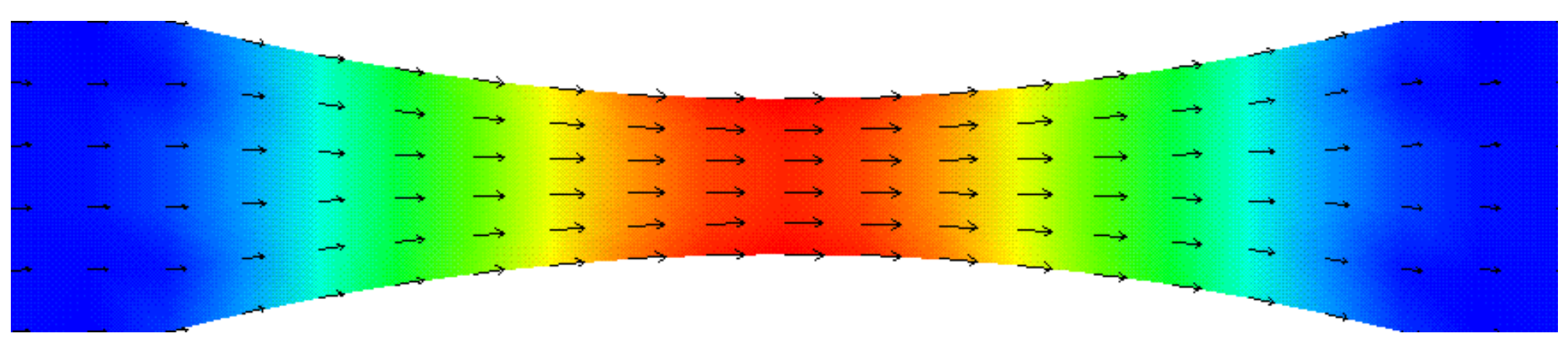

\section{Driven Cavity}

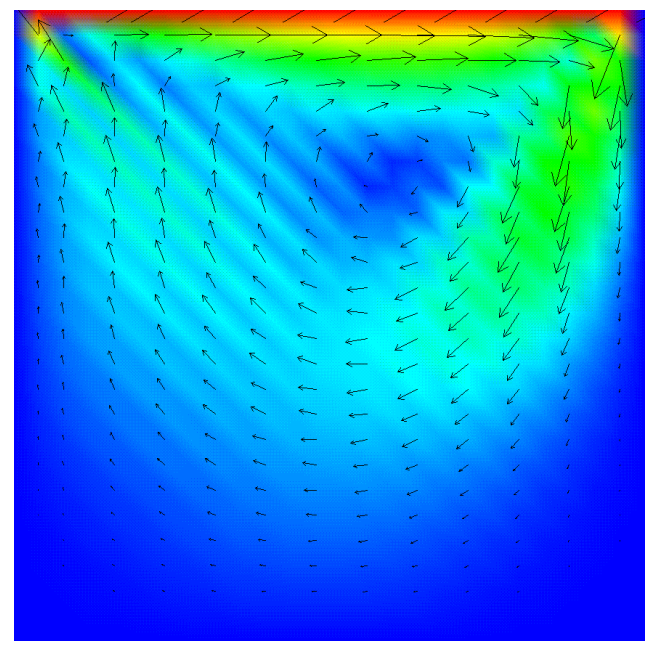

皐 


\section{MULTIPHYSICS MODELING IS ANOTHER PART OF OUR R\&D EFFORT.}

Goals: Flow/Structural/Thermal/Chemistry

Status: Flow/Thermal

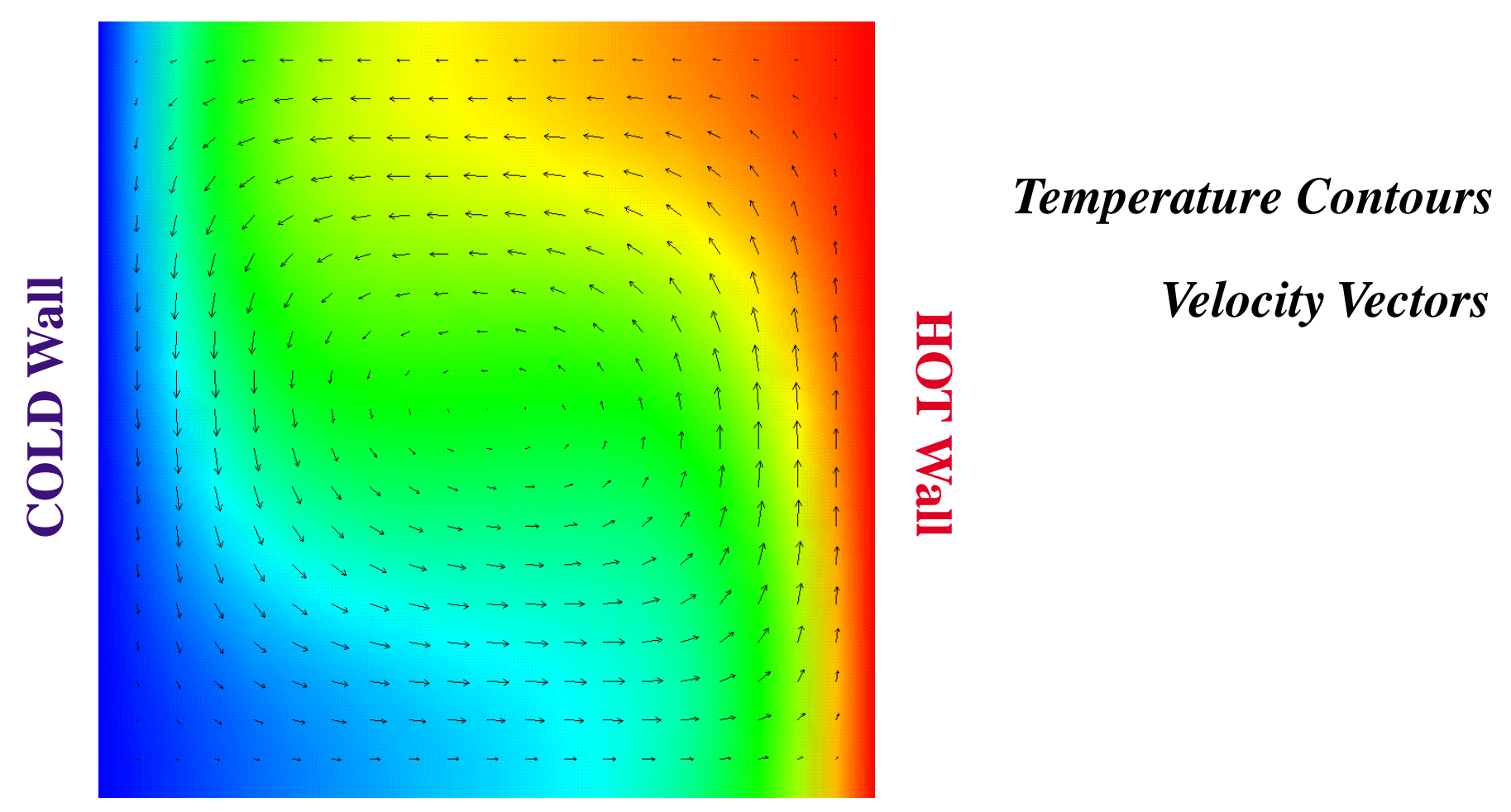

Thermally Driven Cavity 


\title{
Large Eddy Simulation of NASA 7' x 10' Wind Tunnel Tests
}

\author{
Dan Flowers, Rose McCallen, Tim Dunn , Jerry Owens
}

Lawrence Livermore National Laboratory

Livermore, CA

March 16, 1999 


\section{Introduction}

Goal

To develop simulation tools which can accurately predict the flow-field of heavy vehicles. These tools can be applied to investigating drag reduction strategies.

Approach

Carefully validate the simulations with well characterized experimental data

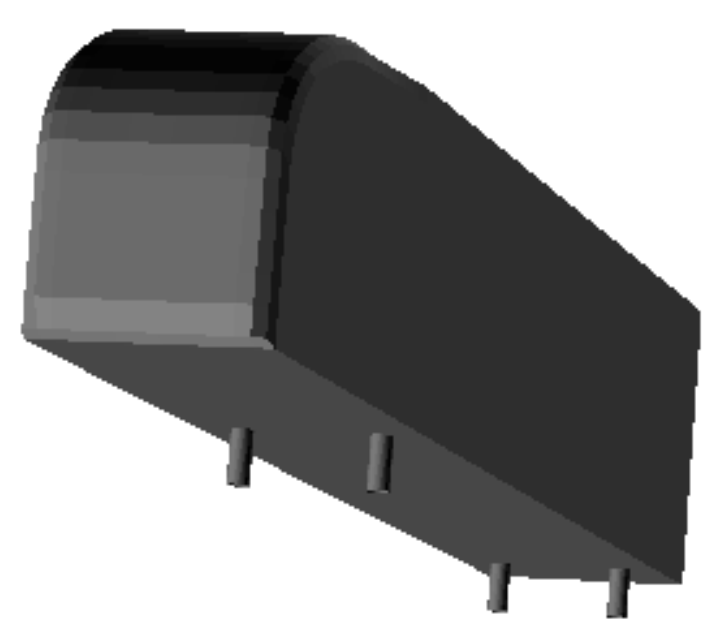




\section{The flow around a truck is difficult to simulate}

Flow is time dependent, 3D with a wide range of scales

Boundary layers transition and separate

Flow tripped by head lamps, grab handles, etc.

Chosen approach

Compressible ALE simulation code

Large-eddy simulation (LES) using the finite element method (FEM)

Captures large scales $>\Delta$ grid

Approximates small scales $<\Delta$ grid

Explicit Scheme $-\Delta t=(\mathbf{u}+\mathbf{a}) / \Delta \mathbf{x}$ 


\section{Several approaches are being used to simulate the GTS}

SNL

Reynolds Average Navier-Stokes (RANS)/ Detached Eddy Simulation (DES)

Compressible Finite Volume Code

Average "Steady" Solution/Unsteady Solution

Widely used - may not predict drag correctly

LLNL

Large Eddy Simulation (LES)

Compressible Finite Element Code

Unsteady Solution of large scales/approximation of the small scales

Computationally intensive

Caltech

Direct Numerical Simulation/ LES

Vortex Method

Gridless

In development 


\section{Turbulent flow contains eddies ranging from large- scale to small-scale}

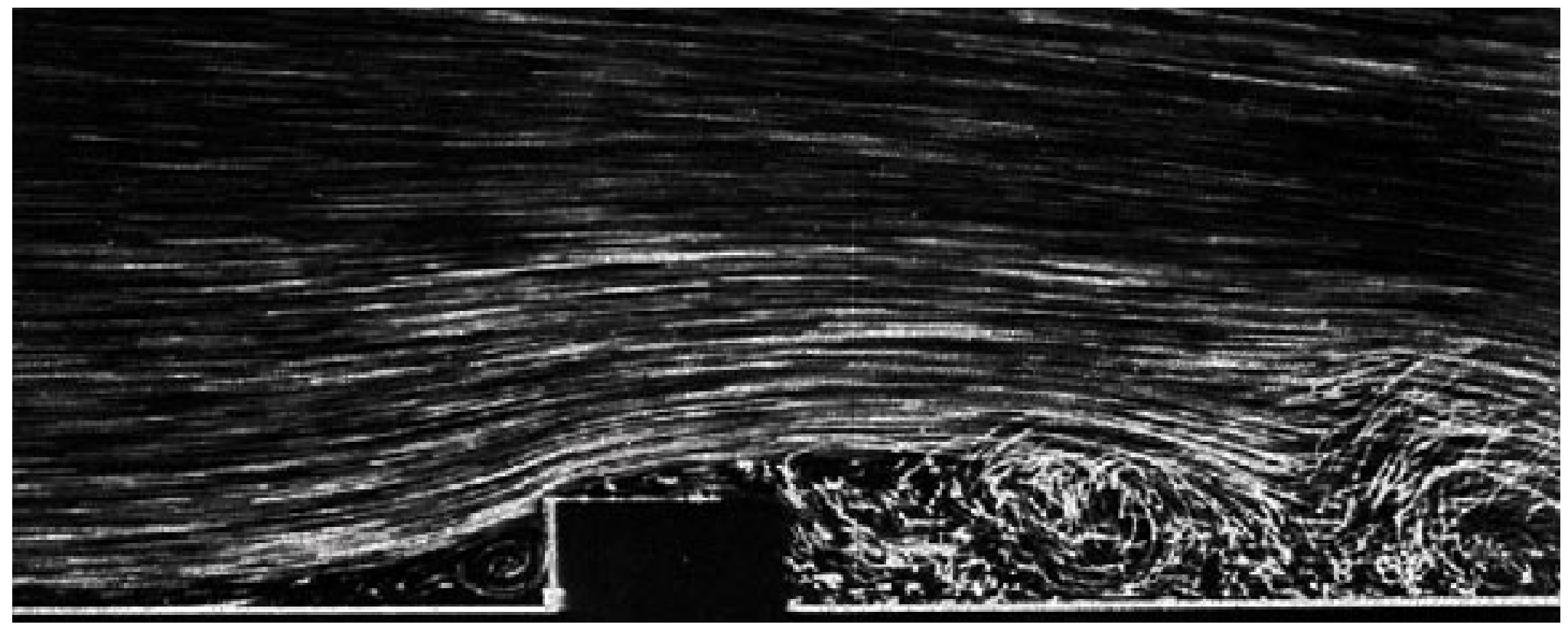

Large-eddy simulation captures the large-scale motion and approximates the small-scale motion.

all turbulent motions $=$ large-scale motions + small-scale motions

$$
\begin{aligned}
& =\text { 'resolved' scale }+ \text { 'subgrid' scale } \\
\mathrm{u}_{\alpha} & =\overline{\mathrm{u}}_{\alpha}+\mathrm{u}_{\alpha}^{\prime}
\end{aligned}
$$




\section{Streamwise Velocity}

LES: instantaneous and/or time-averaged with 1 empirical parameter

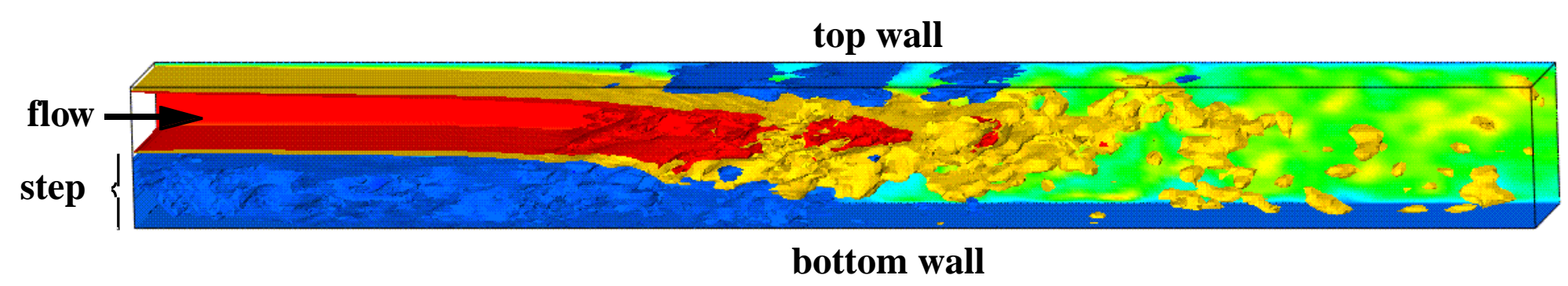

RANS: only time-averaged with many empirical parameters

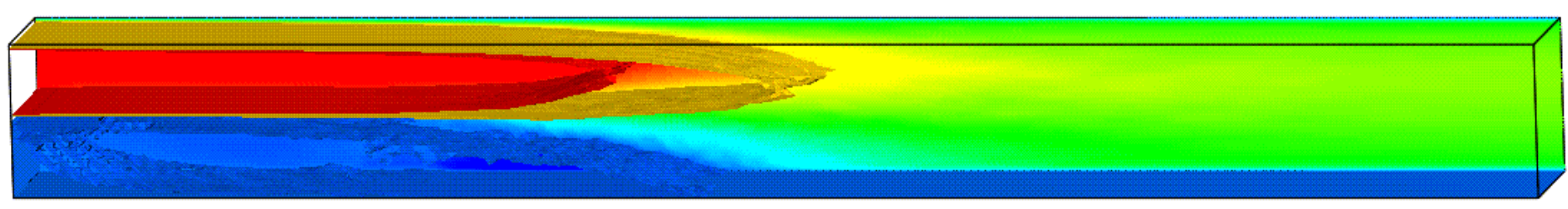




\section{Modeling the unsteady, 3-D vortex shedding in 'step-flows' requires innovation}

Commercial state-of-the-art

Reynolds-Averaged Navier-Stokes (RANS) turbulence model

Many empirical parameters

2D, steady, time-averaged solution

Backward-facing step: streamwise velocity

top wall

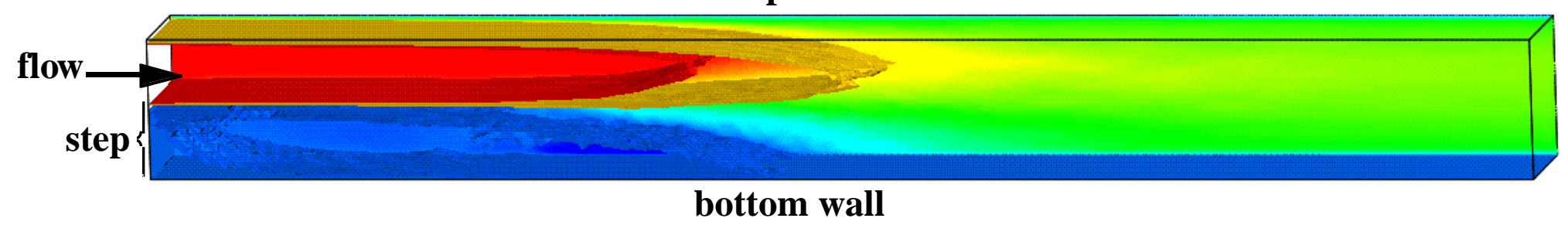

Current leading-edge technology

Large-eddy simulation (LES) turbulence model

One empirical parameter (maximum)

3D, unsteady solution of vortex shedding

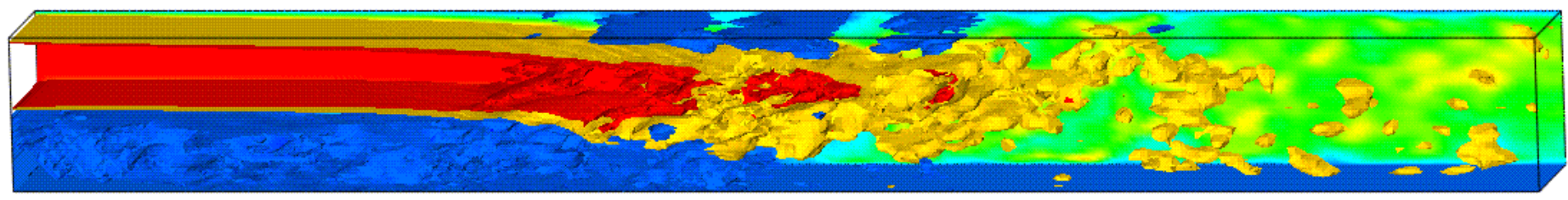




\title{
The LES challenges are related to physical as well as numerical modeling.
}

Boundary layers are very thin Can't resolve boundary layers - problem gets too big Wall approximations in development

Runtime very long

Evolution is over long time scales

Parallel computations/solvers required - in development

Analysis

\author{
Huge data sets
}

Visualization required - in development

Methods for testing convergence $(\mathrm{V} \& \mathrm{~V})$ in development

Significant development being done by LLNL programs. 
NASA wind tunnel tests have been conducted on a simplified truck geometry

- NASA experimental data available for several yaw angles (from $0^{\circ}$ to $15^{\circ}$ )

- Simulations to be validated with experimental data

Flow

Direction
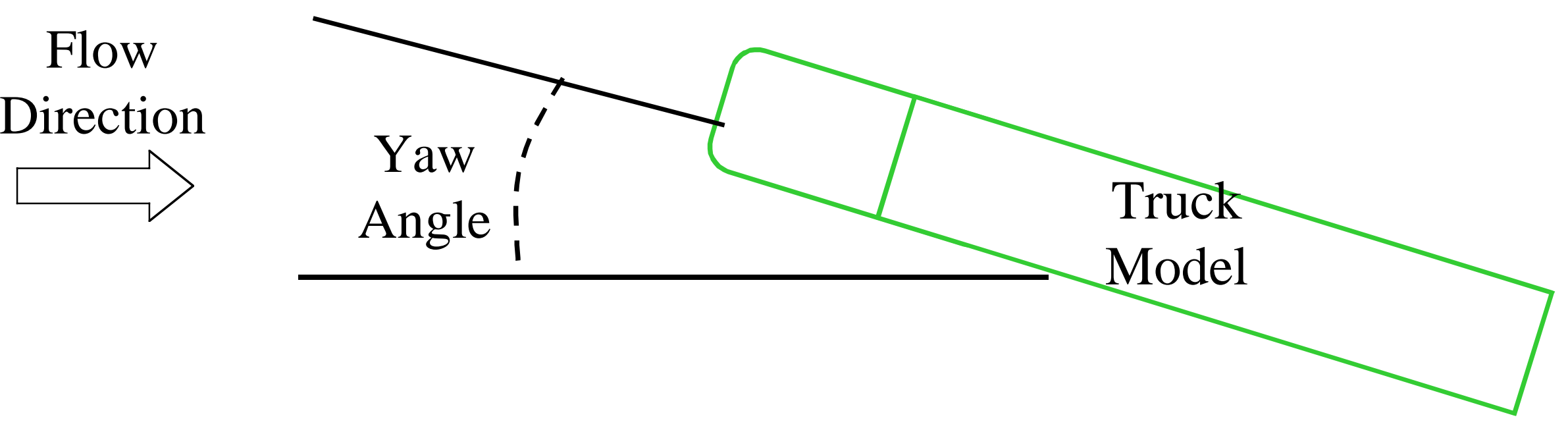


\section{Simulations focus on NASA 7'x10' wind tunnel tests}

A Grid of the full 7'x10' wind tunnel has been developed

- Scaleable

- Allows for yawing of the model

Currently simulating involves $0^{\circ}$ yaw to validate model and study mesh refinement

Compressible/Incompressible simulations for $0^{\circ}$ yaw

\subsection{Million Completed}

2.4 Million In progress

19 Million Future

154 Million Future 


\section{3 million element mesh}
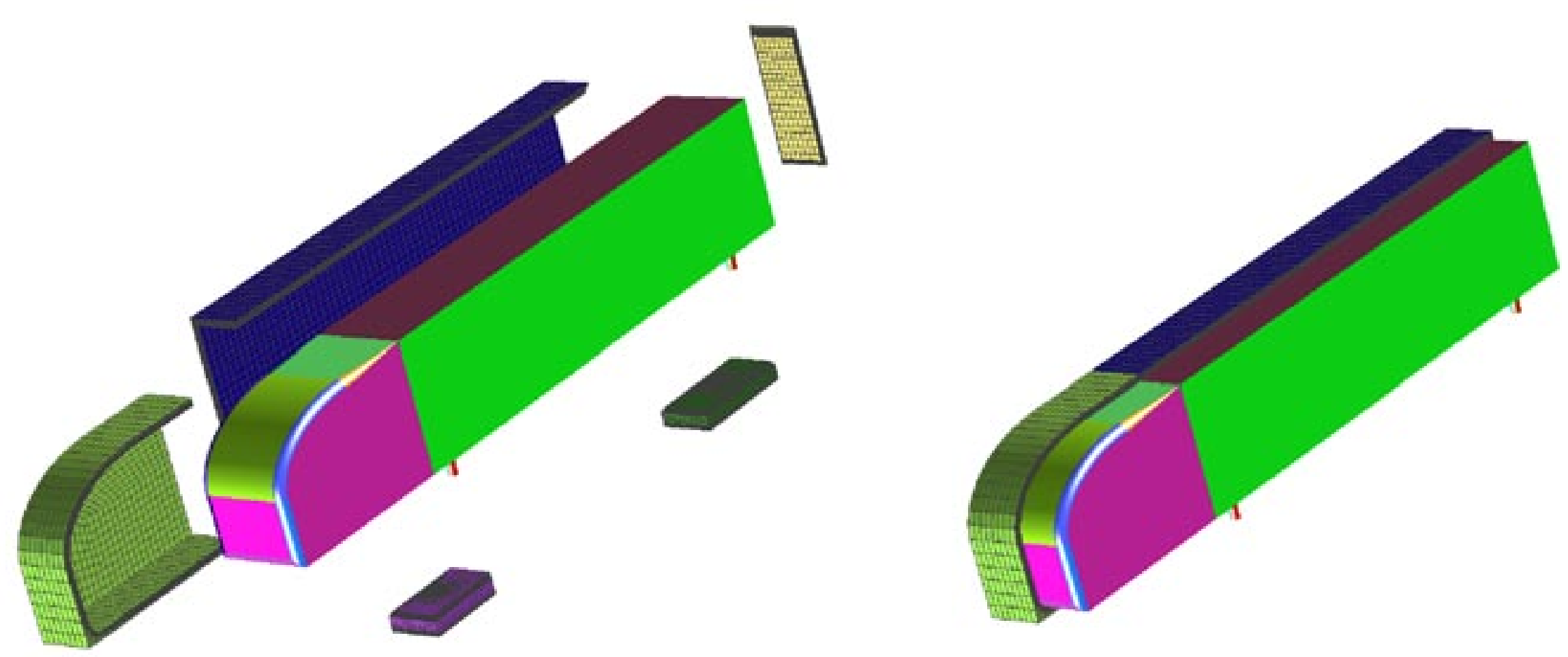

- Unstructured multi-block mesh

- Near body mesh can be refined independently of "Far-Field" mesh

- Full geometry simulated - mesh reflected about symmetry plane 


\section{3 million element mesh}
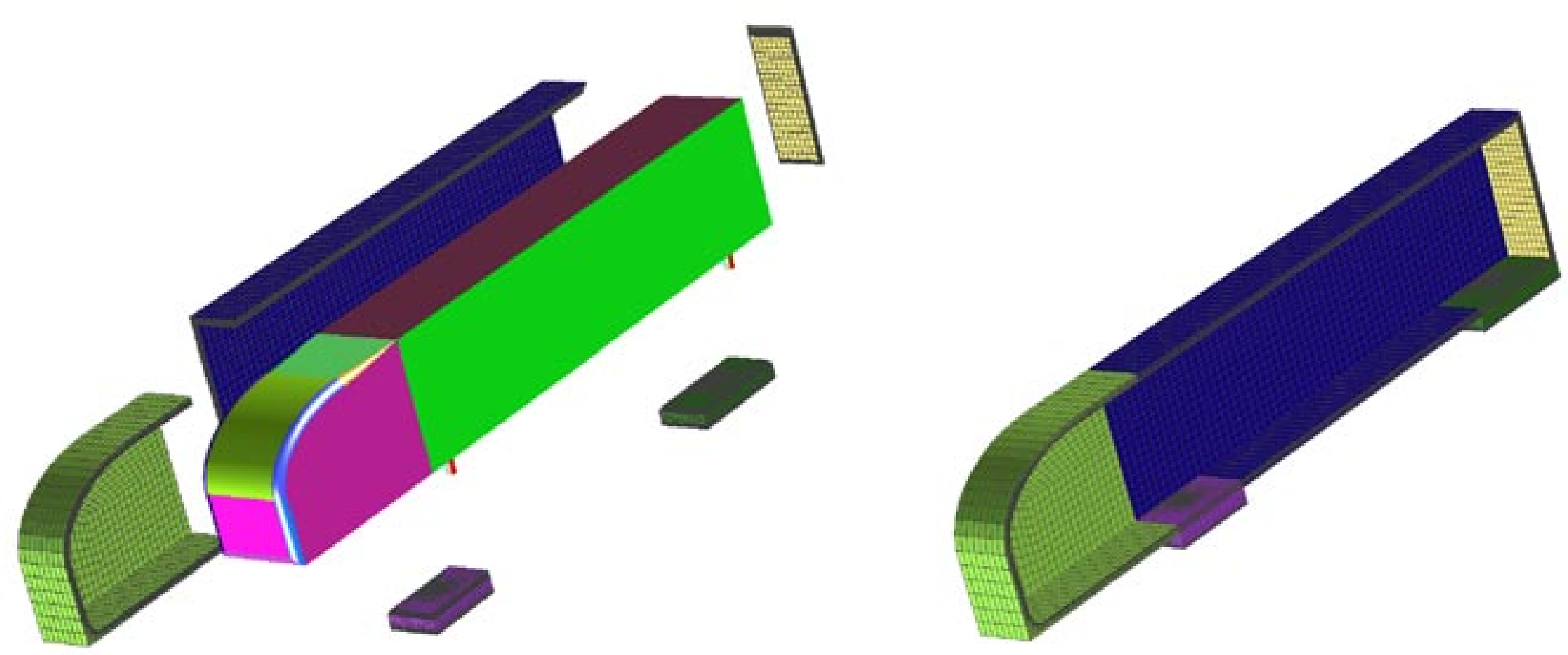

- Unstructured multi-block mesh

- Near body mesh can be refined independently of "Far-Field" mesh

- Full geometry simulated - mesh reflected about symmetry plane 
0.3 Million Element Mesh

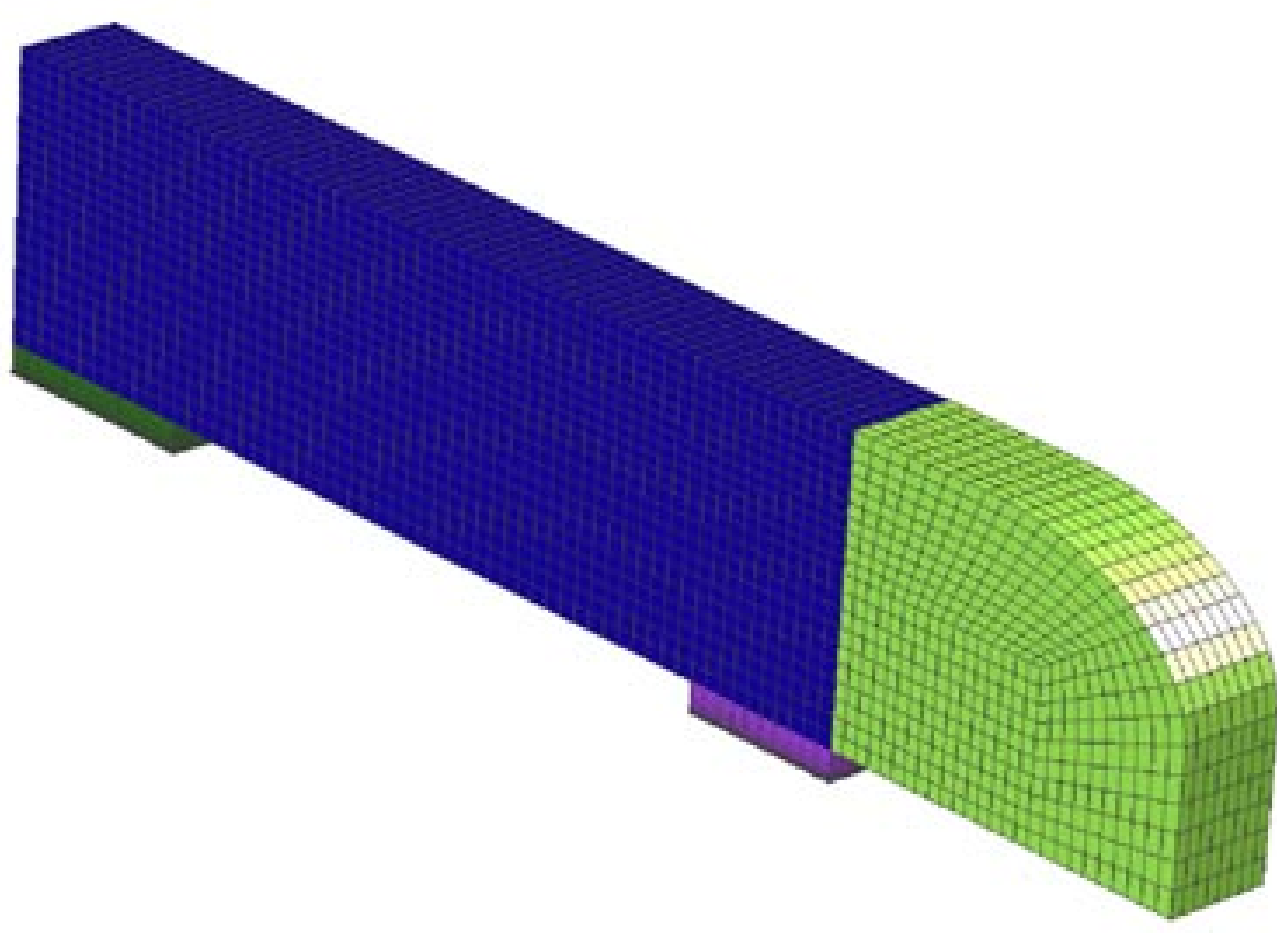


0.3 Million Element Mesh

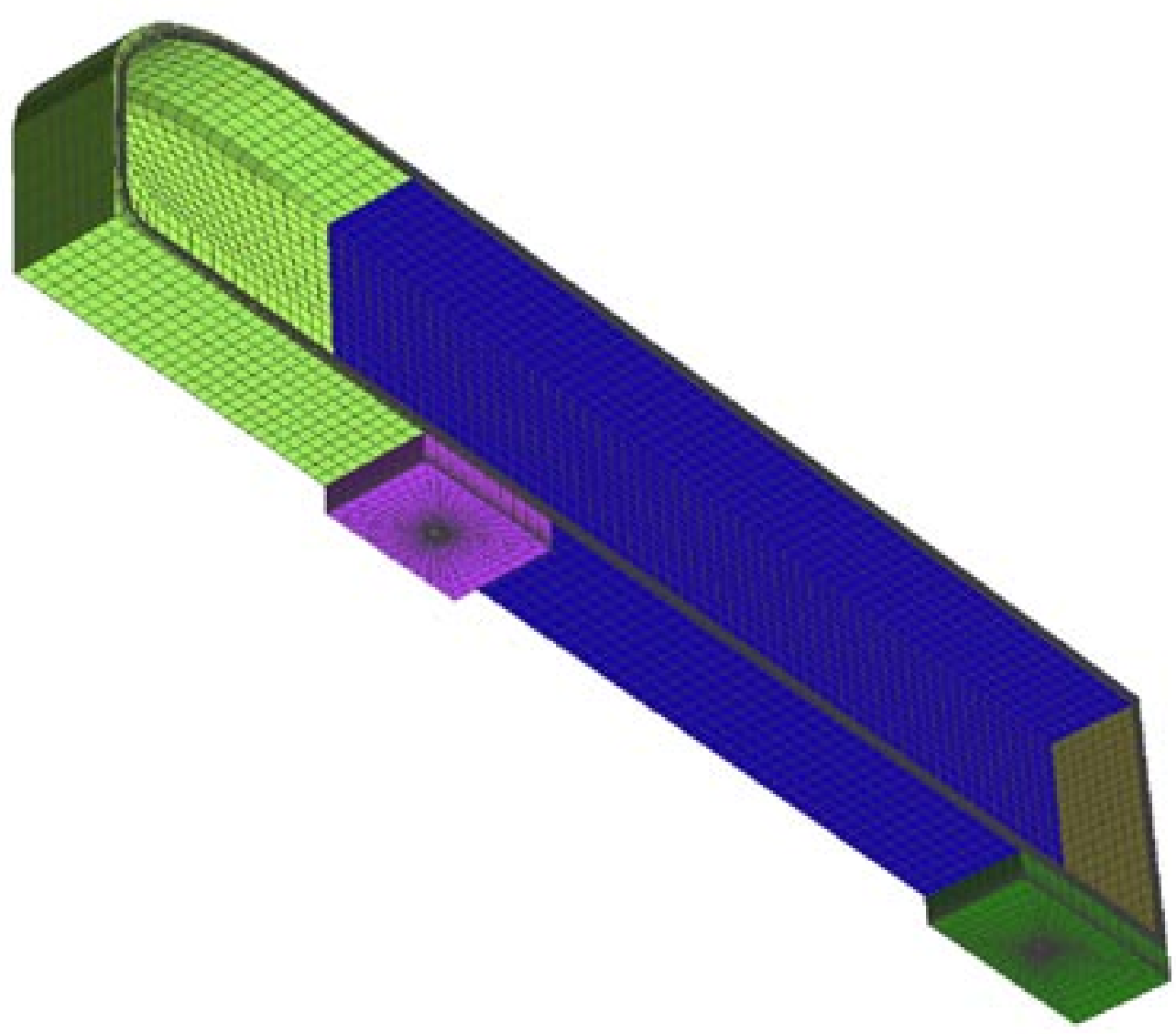


0.3 Million Element Mesh
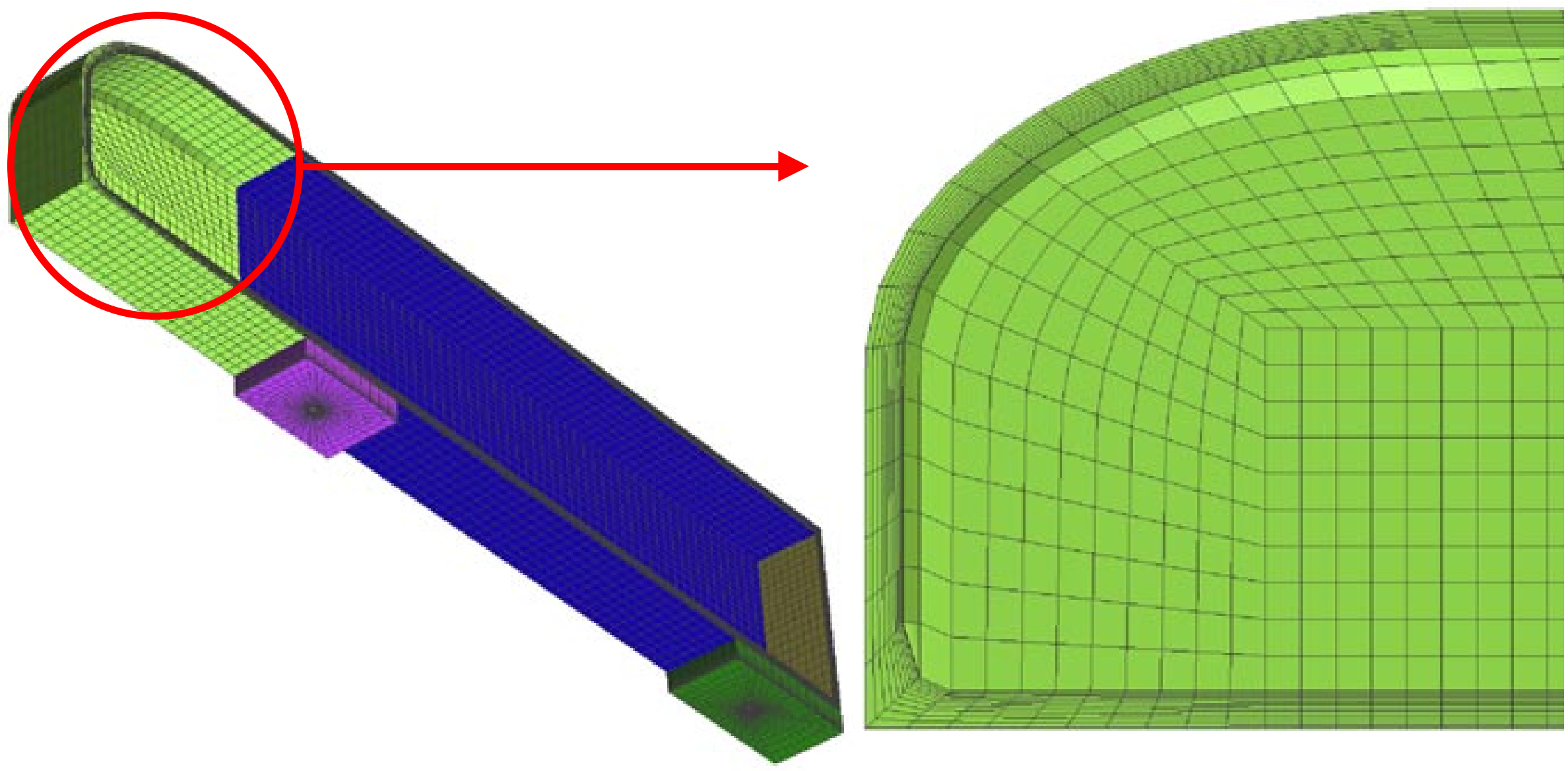


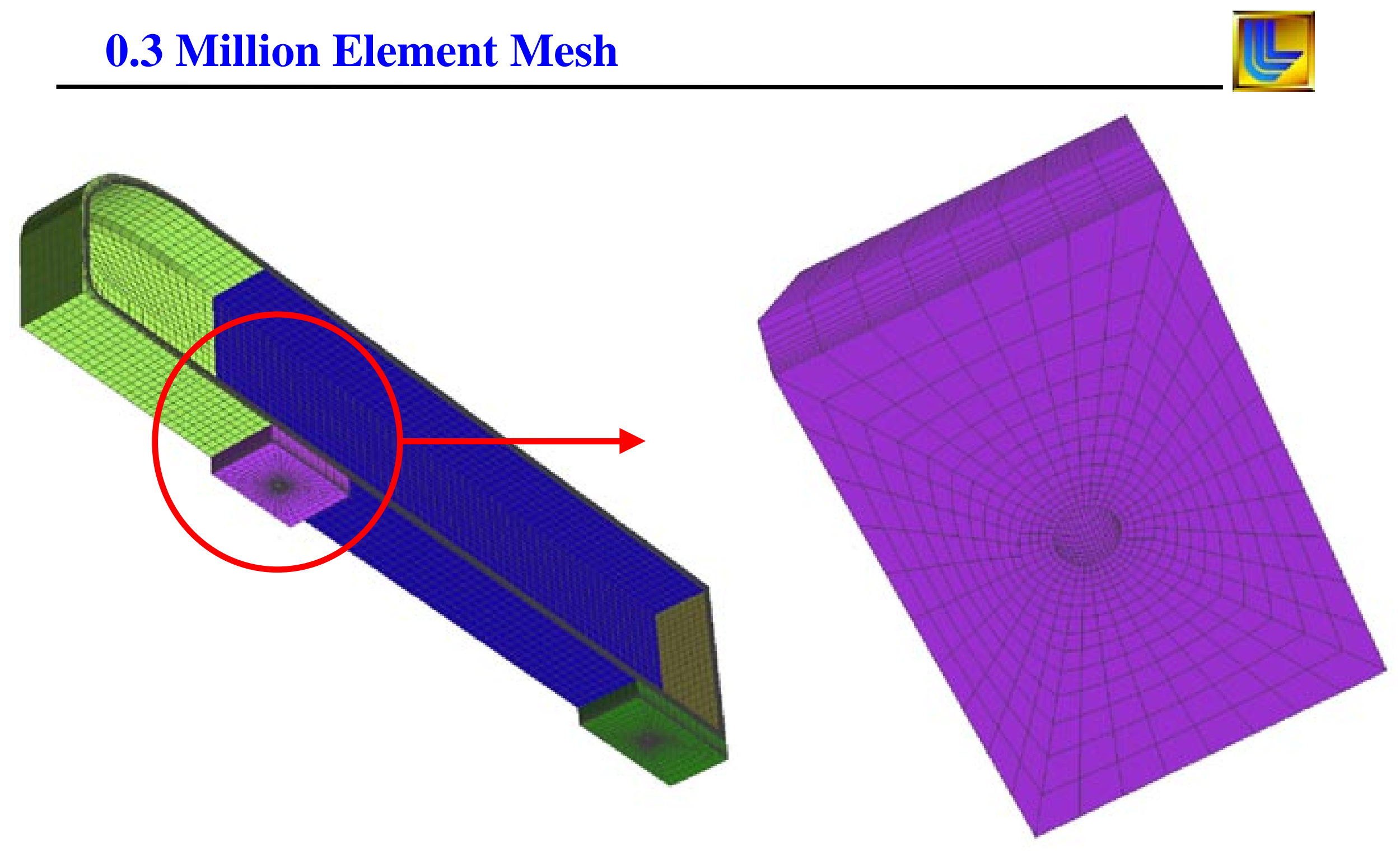


0.3 million element mesh

나 
0.3 million element mesh

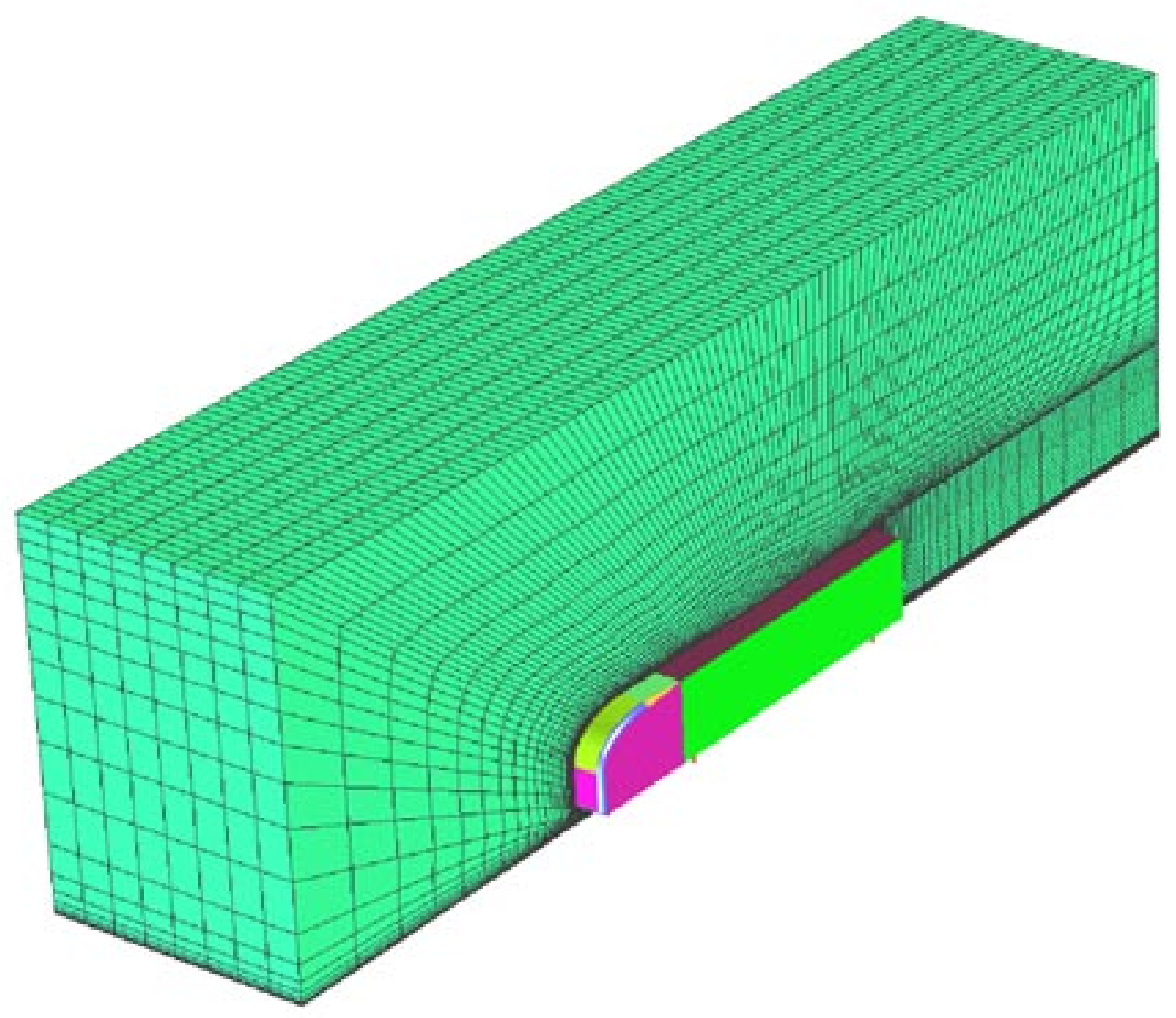




\section{Parallel computation}

- Parallel computation necessary because of large number of elements

- Domain decomposition performed using parmetis algorithm

- Simulations are being performed on massively parallel ASCI Blue machine

- 0.3M Element Mesh (24 processors)

- 2.4M Element Mesh (148 processors)

- 19M Element Mesh ( 1000 processors)

- 154M Element Mesh ( 5000 processors)

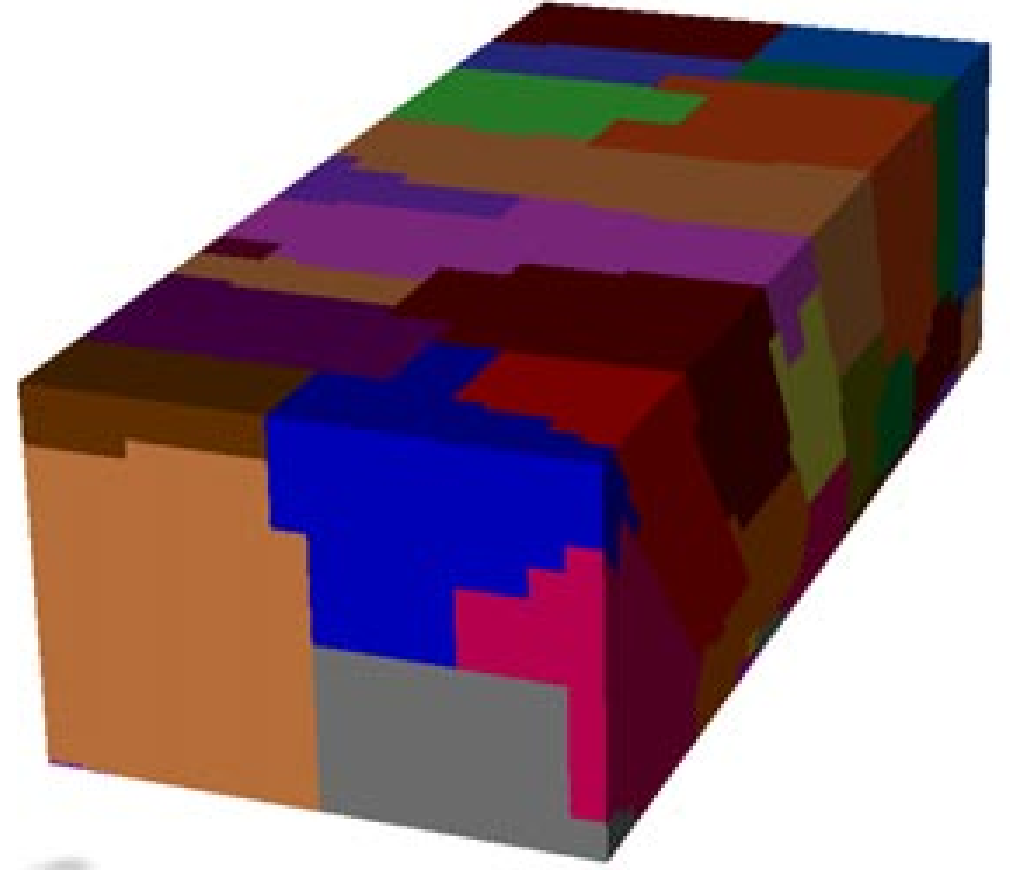

Domain decomposition for 0.3 million element 


\section{Simulation results - drag coefficient}

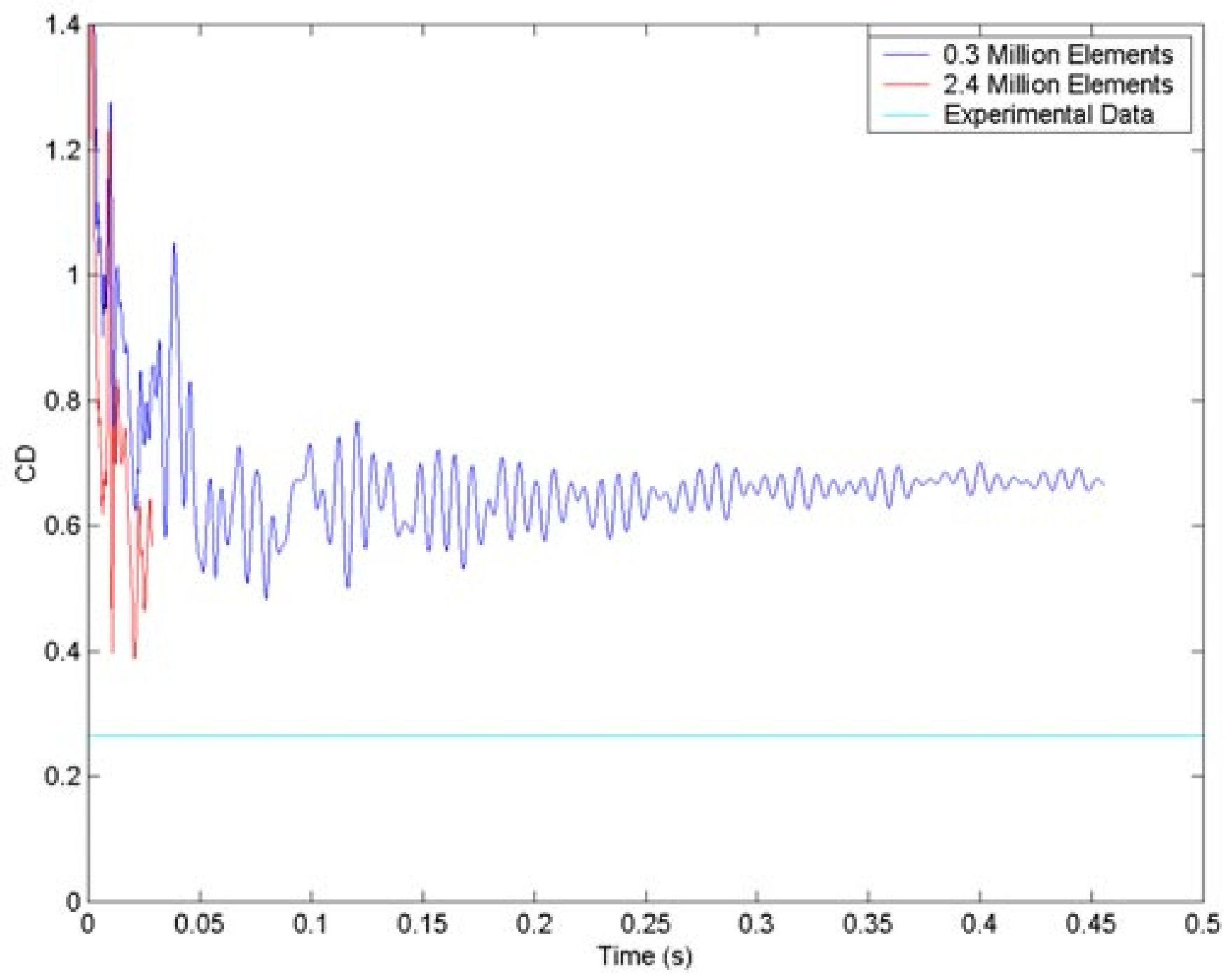


Simulation results - drag coefficient

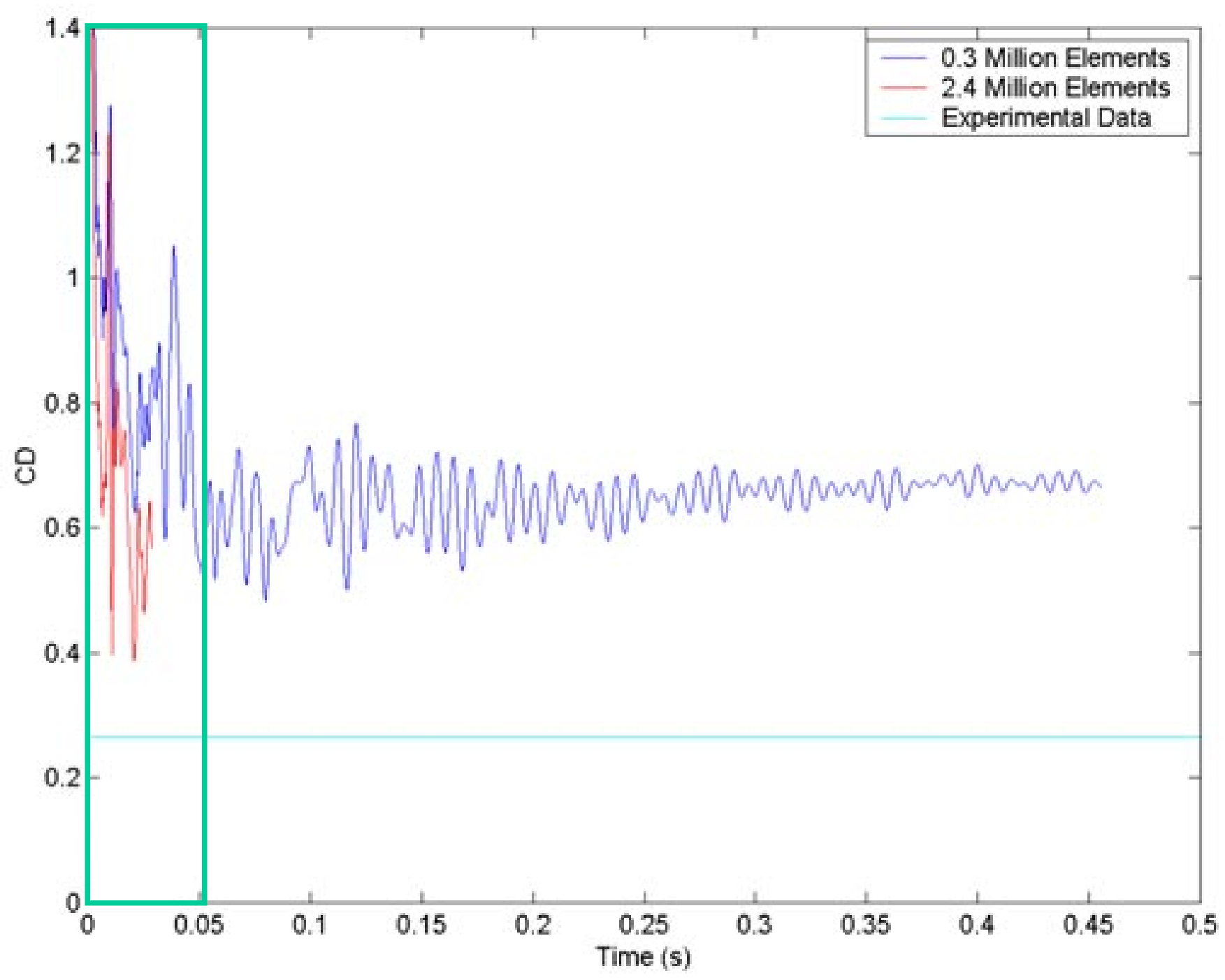




\section{Simulation results - drag coefficient}

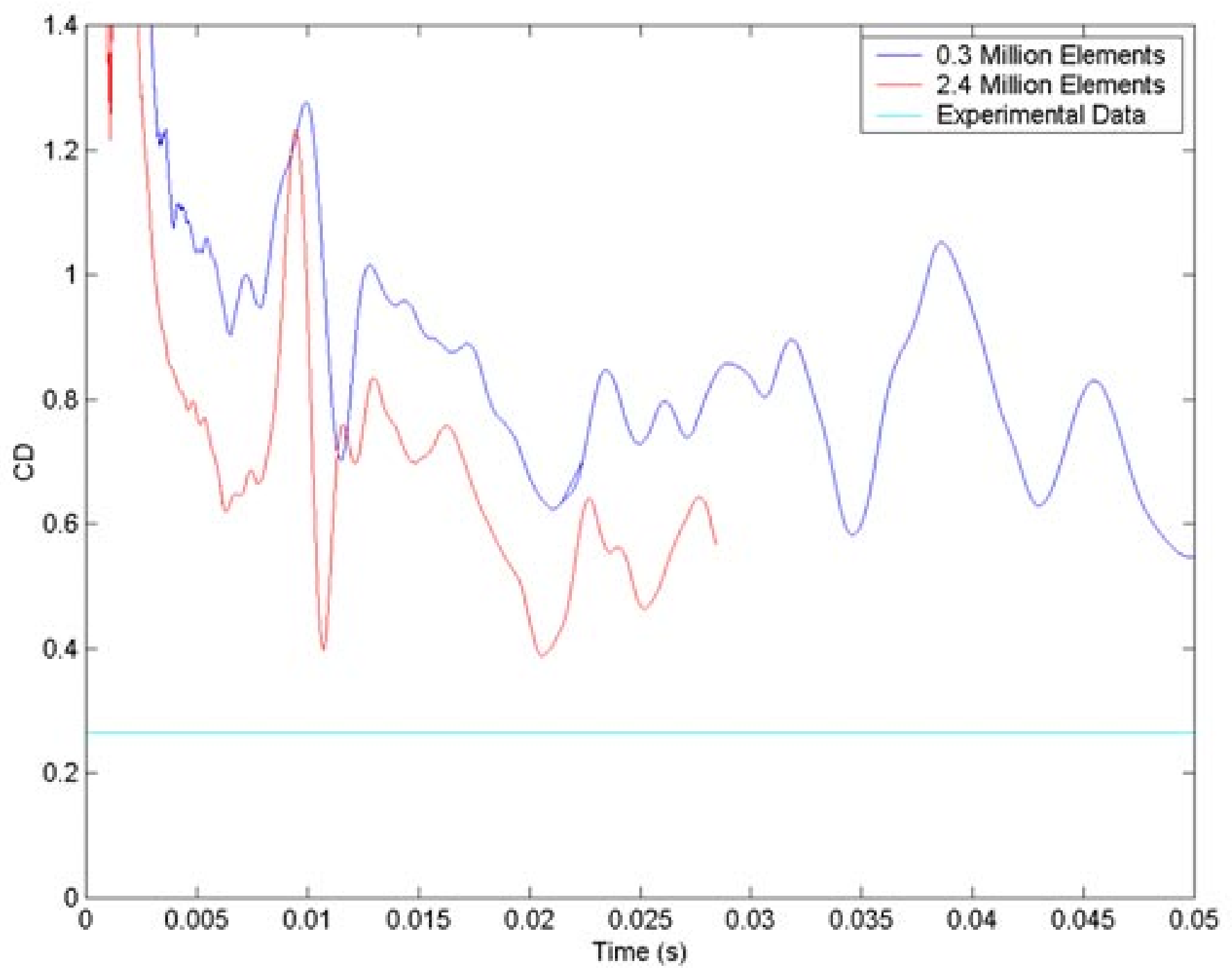


Simulation results - unsteady pressure measurement

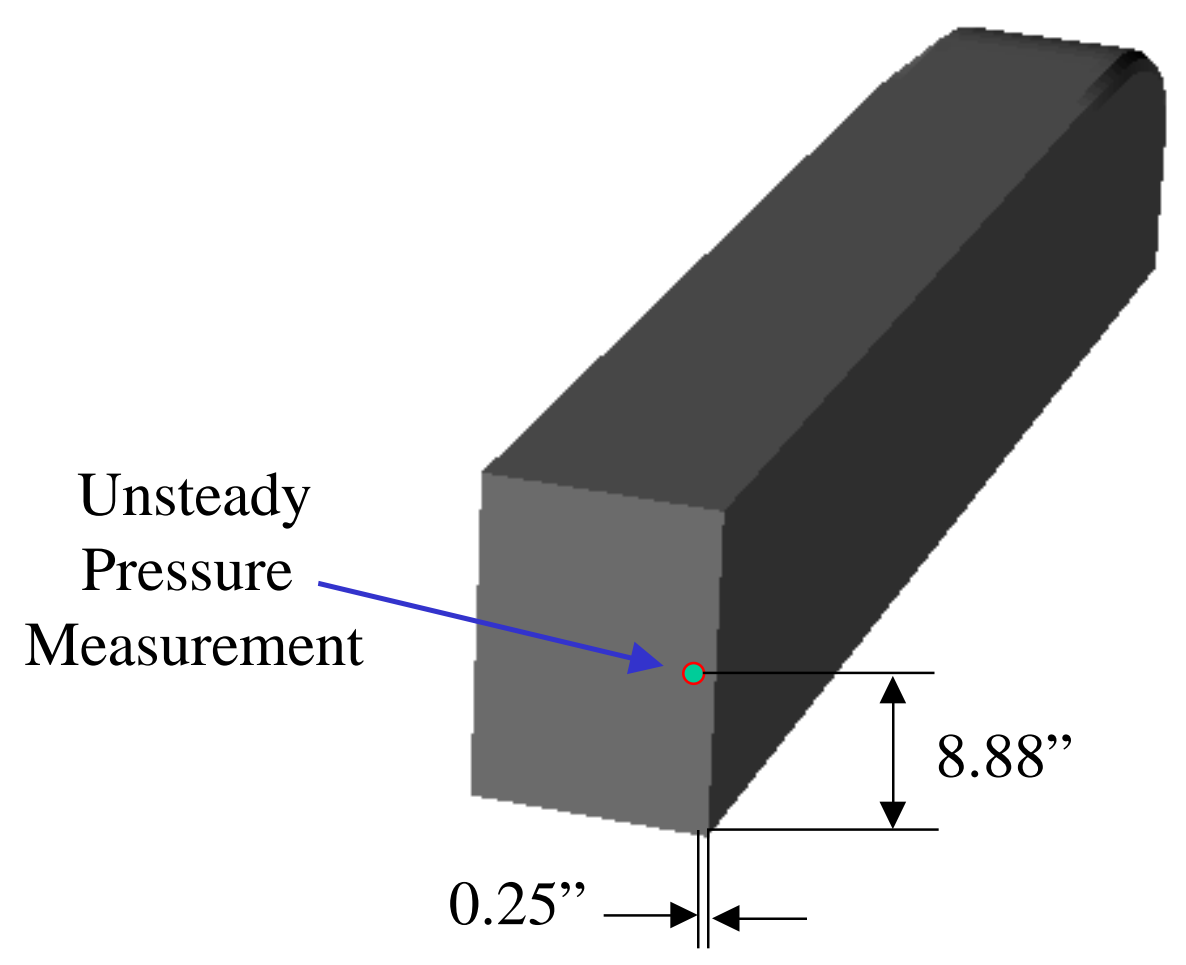

0.3 Million Element Mesh 
Simulation results - unsteady pressure measurement

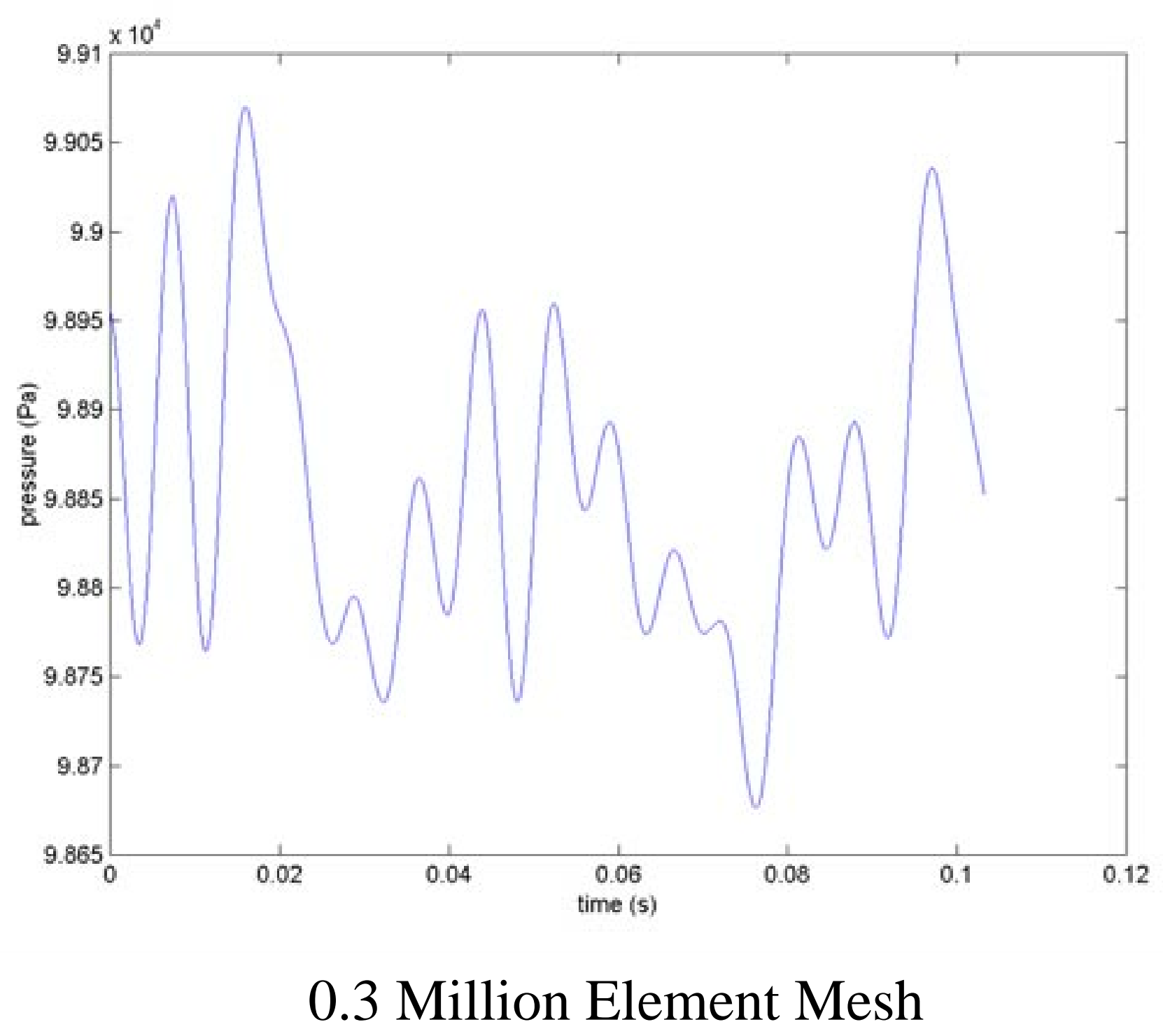


Simulation results - unsteady pressure measurement

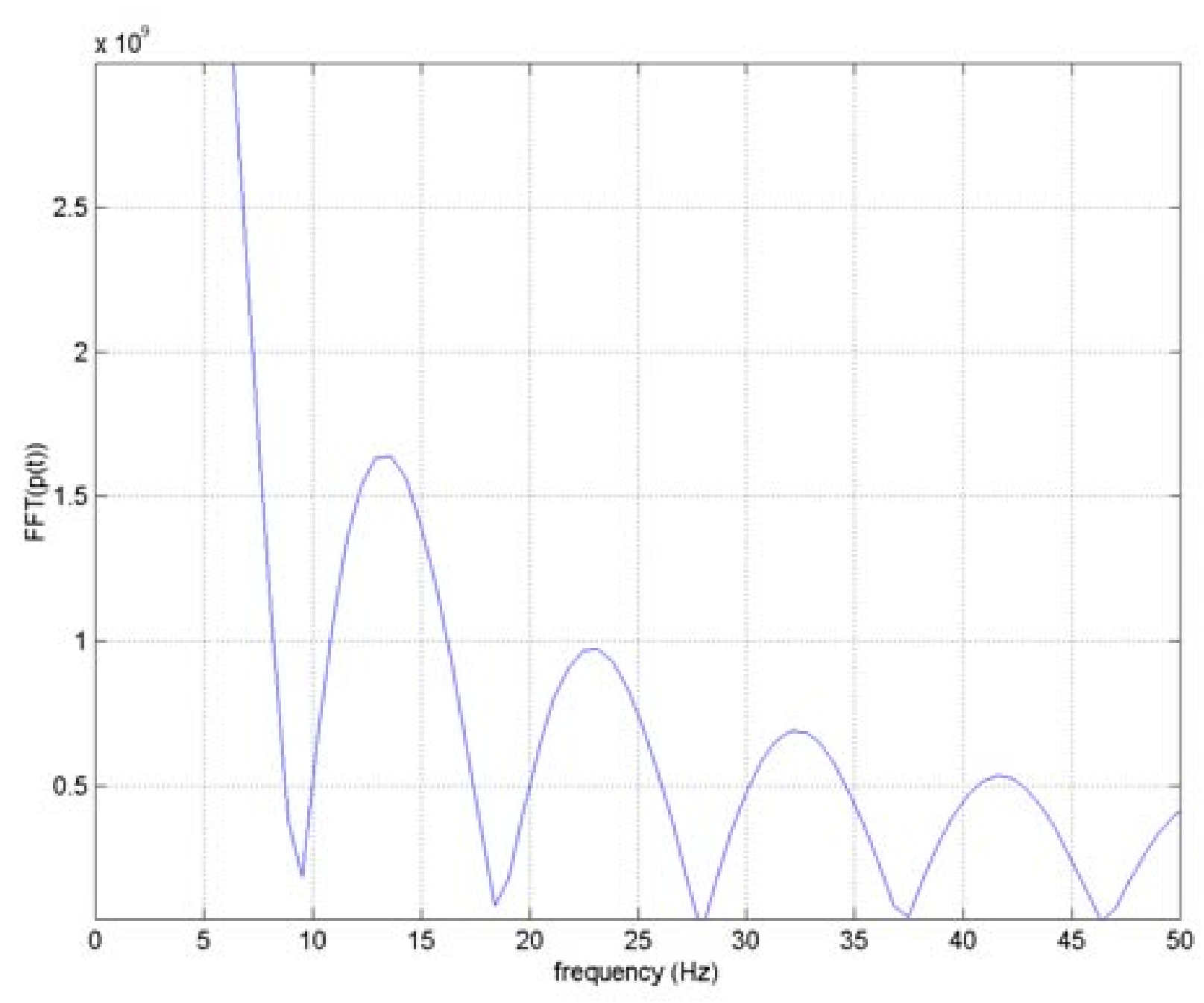

0.3 Million Element Mesh 
Simulation results - time average flow field 
Simulation results - time average flow field

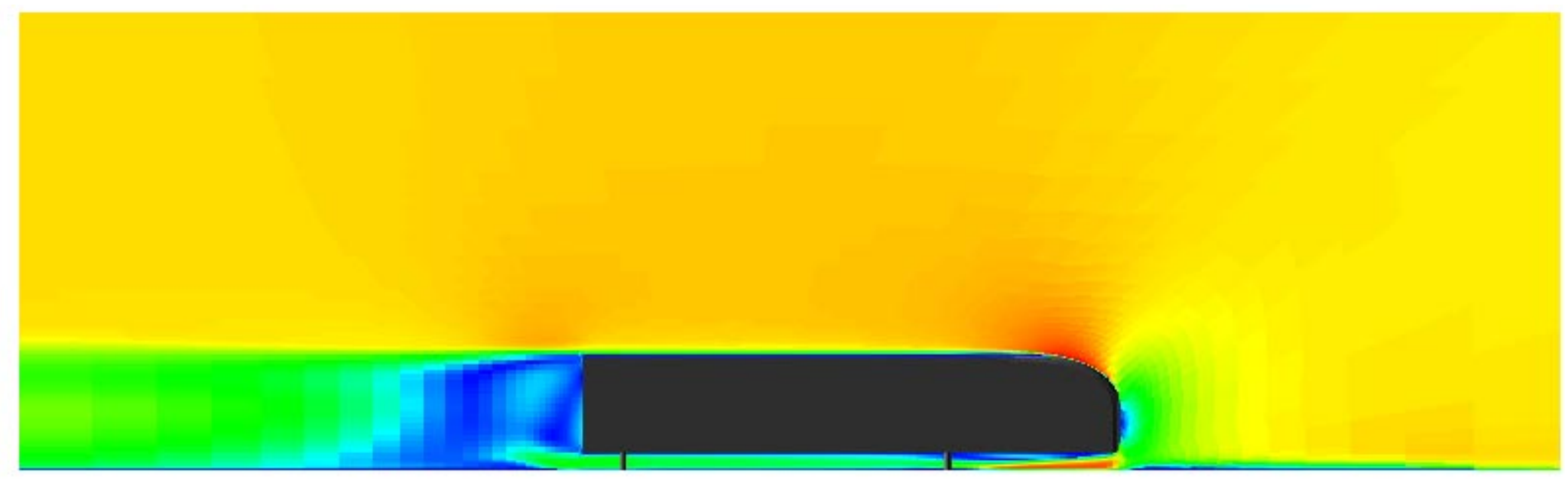


Simulation results - time average flow field

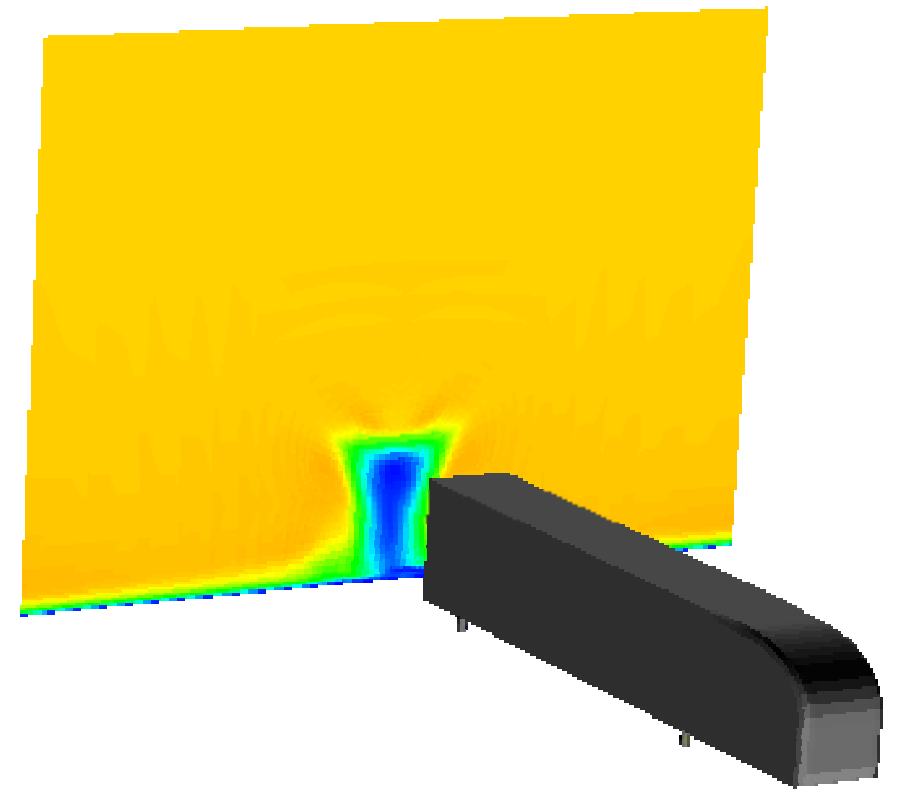

$3 \mathrm{~m}$ from front of truck 
Simulation results - time average flow field

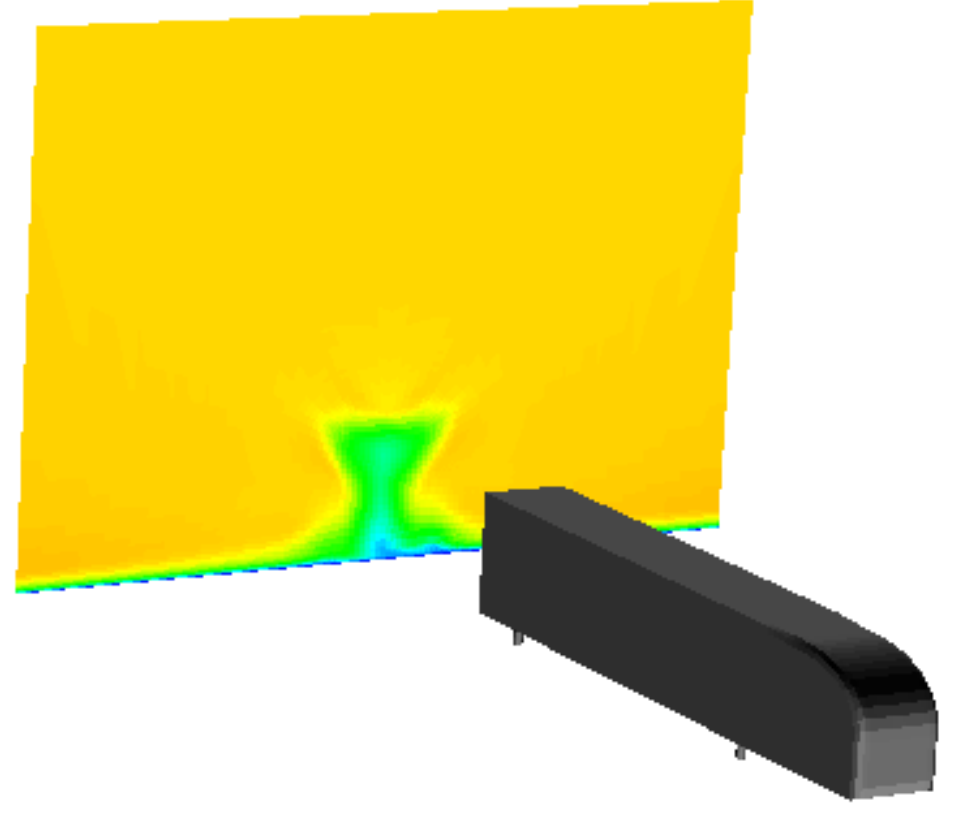

$3.5 \mathrm{~m}$ from front of truck 
Simulation results - time average flow field

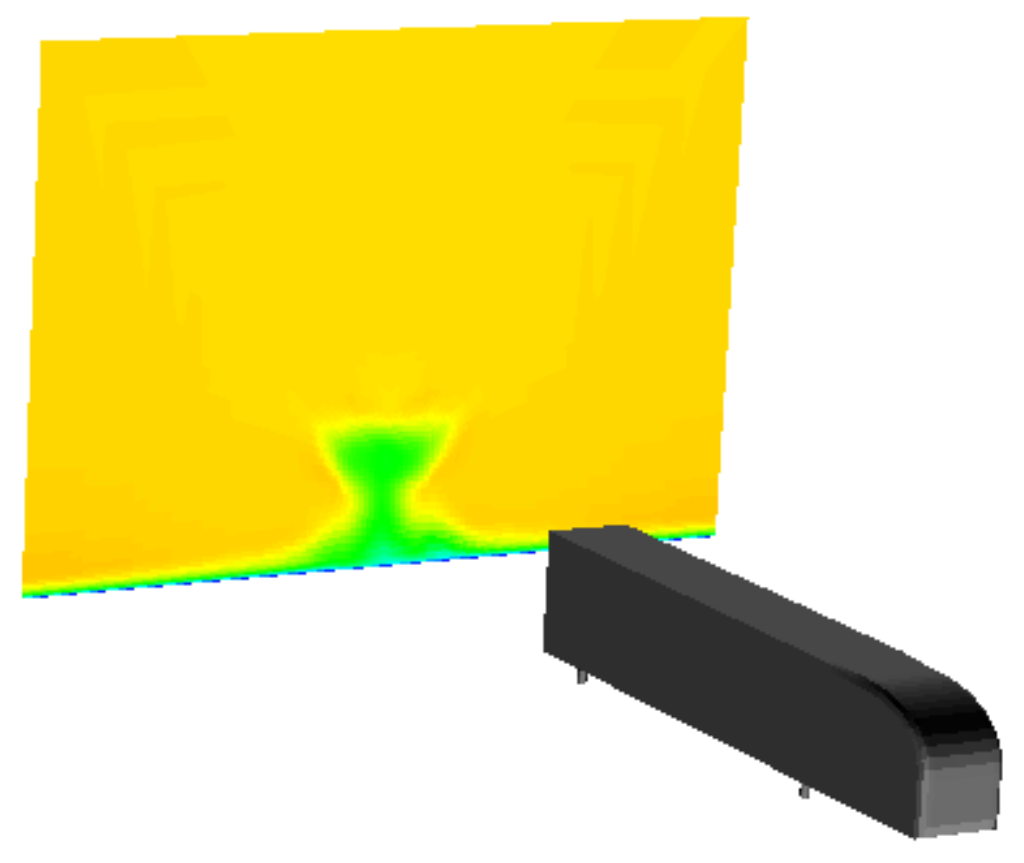

$4 \mathrm{~m}$ from front of truck 


\section{Simulation results - movie}

DE: truekn-144. .0050240

T,me: 0.0675009 CYele: 50240

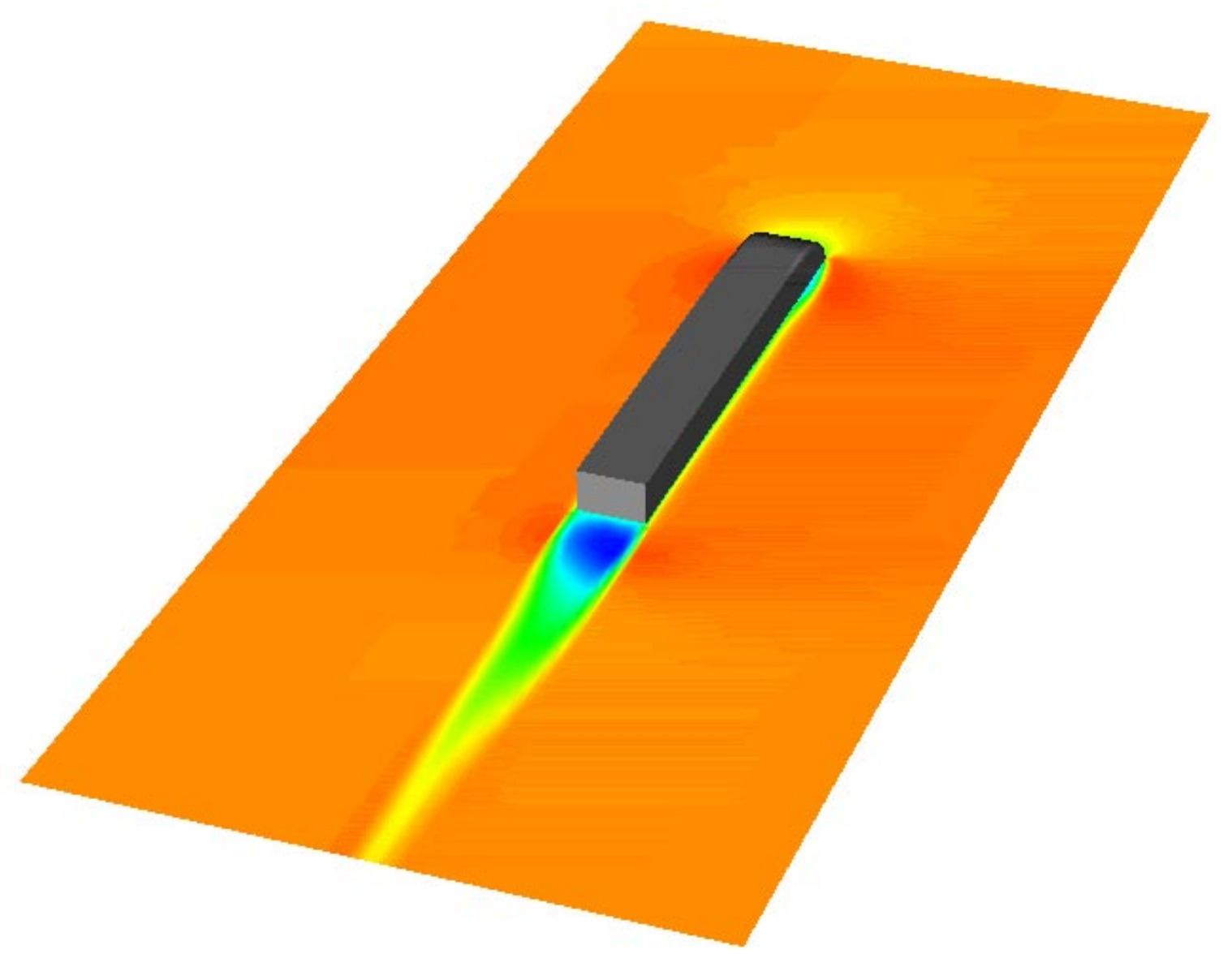


A scalable grid has been created for the NASA 7'x10' wind tunnel geometry

Compressible simulations for $0^{\circ}$ yaw with 0.3 Million and 2.4 Million Element mesh in progress 


\title{
Simulation of Complex, Unsteady Flows Using a Grid-Free Vortex Method
}

\begin{abstract}
A. Leonard
Graduate Aeronautical Laboratories

California Institute of Technology
\end{abstract}

M. Brady, L. Barba, M. Rubel 


\section{Vortex Code: Improvements}

- Surface mesh adaptivity - triangles no longer restricted to particle size

- High order surface interpolants in progress

- Vortex particle size mapping function nearly complete

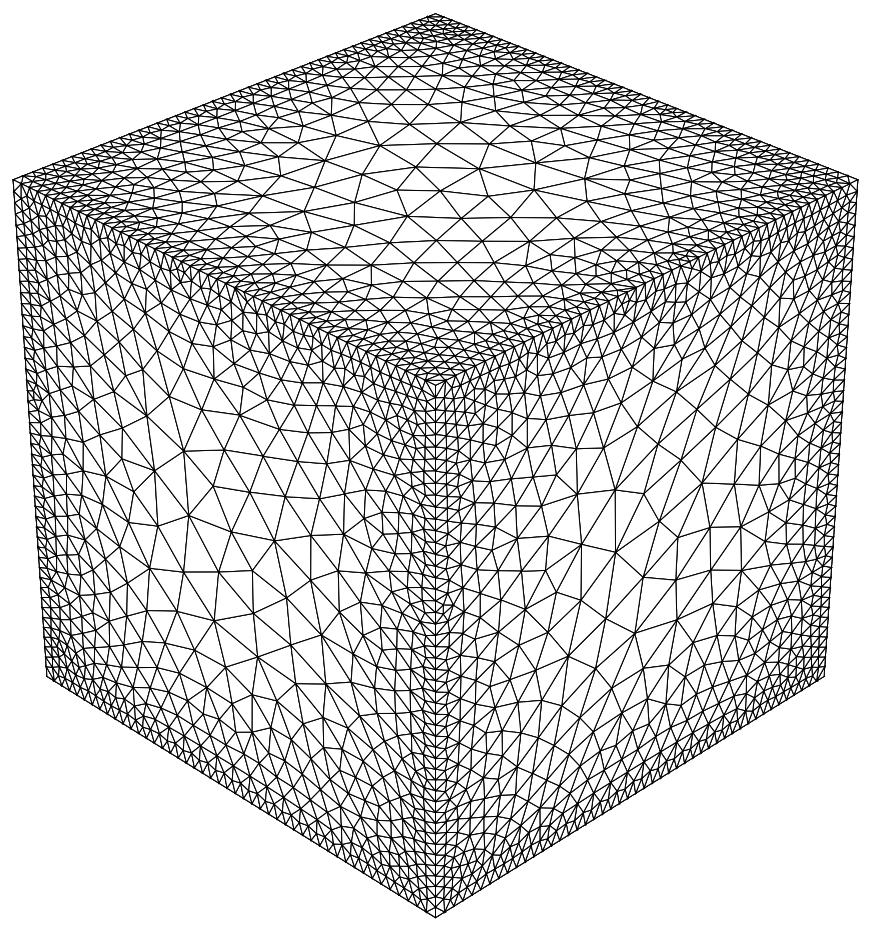




\section{Vortex Code: Results}

- Definition of GTS-scale geometry

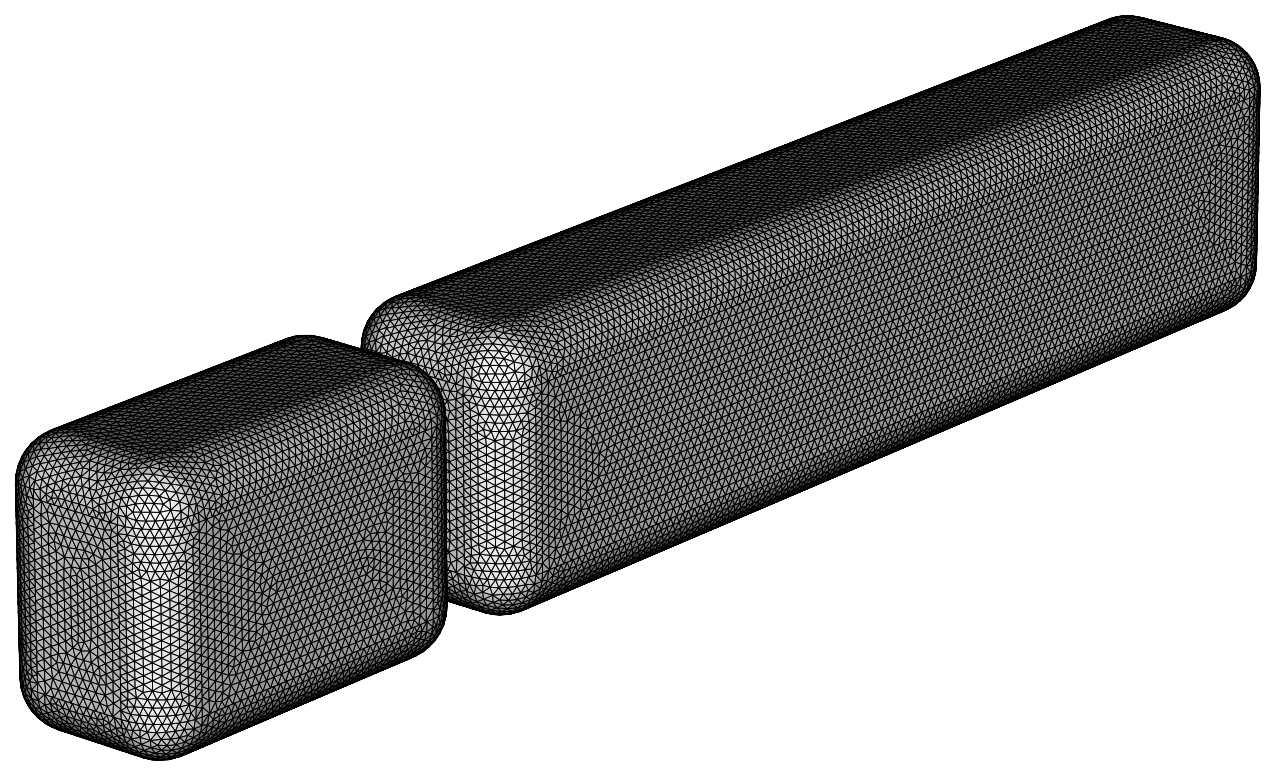

- Tandem rounded cube (animation)

$R e=100$

- Tandem rounded prism at GTS-scale (animation) $R e=100$ 


\section{Vortex Code: Verification}

- Drag calculation via differentition of fluid impulse

$$
C_{D} \propto \frac{d}{d t} \int_{V} x \times \omega d V
$$

- Drag curves for sphere, rounded cubes, $R e=100$

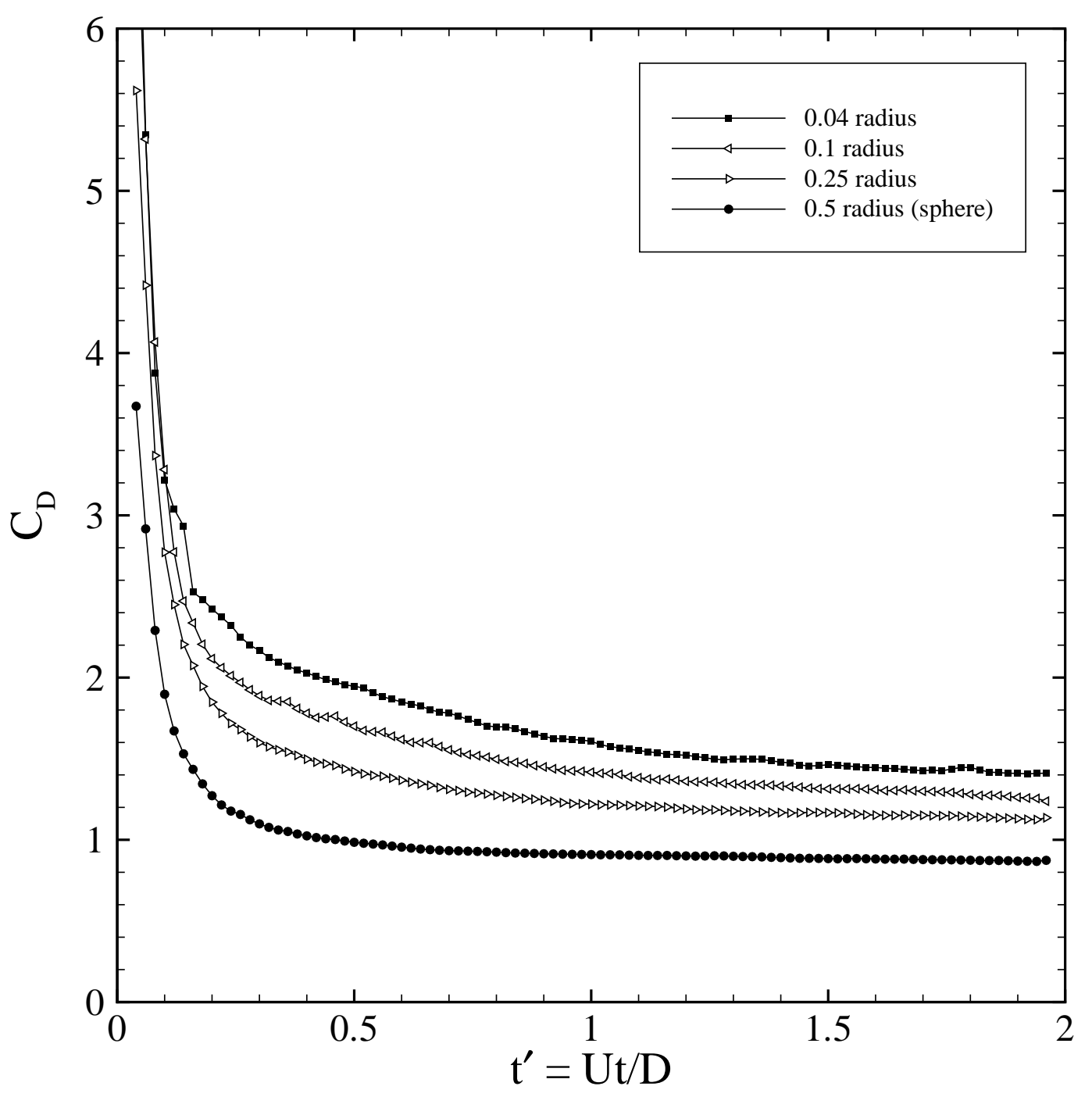




\section{Large-eddy Simulation: Problem of High Re Wall Turbulence}

- Length-scale near wall = Sqrt(viscosity/velocity gradient $)$ - wall unit

- Large eddies near wall O(10 - 100) wall units

- LES grids 40 x 10 x 10 wall units near wall

- Pipe flow $\mathrm{Re}=3 \times 10 * * 4 \Rightarrow \mathrm{R}+=800$

\# LES grid volumes $=5 \times 10 * * 5$

Pipe flow $\mathrm{Re}=3 \times 10 * * 7 \Rightarrow \mathrm{R}+=550,000$

\# LES grid volumes $=5 \times 10 * * 11$

\# grid volumes proportional to $\mathrm{R}+* * 2=\mathrm{Re} \_t a t^{*} * 2$ 


\section{Wall layer SGS Models}

- RANS for attached turbulent boundary layers, LES for wakes => DES (Spalart et al 1997)

- RANS for near-wall layer

LES for outer turbulent boundary layer plus wakes

- Above with stochastic forcing of outer layer (LES or planetary boundary layers, Mason \& Thomson, 1992) 


\section{Status / Future Work}

- Incorporation of GTS model into full Vortex Method

- Implementation of the Vortex Method for arbitrary complex geometries

- Analysis of Reynolds number effects (leading edge curvature)

- Subgrid stress model for Large-Eddy Simulation 


\section{Ross Sheckler}

\section{Dynacs Engineering Co., Inc.}


Industry Buy-in?

PACCAR J. Bectold-kw, C. Brewster -PB, Keith Howard-Leyland, Martin de Loww-DAF, Bob Morrison. PTC

Freightliner Luis Novae, Gene Baron,...

- USE tA

- go after application of what we knOW - LEG GAL HURDLES
HURDLES TECH BENEFITS AFTER LEGAL
HOR

-PROTOTYPING COST $\angle \$ I M$ 


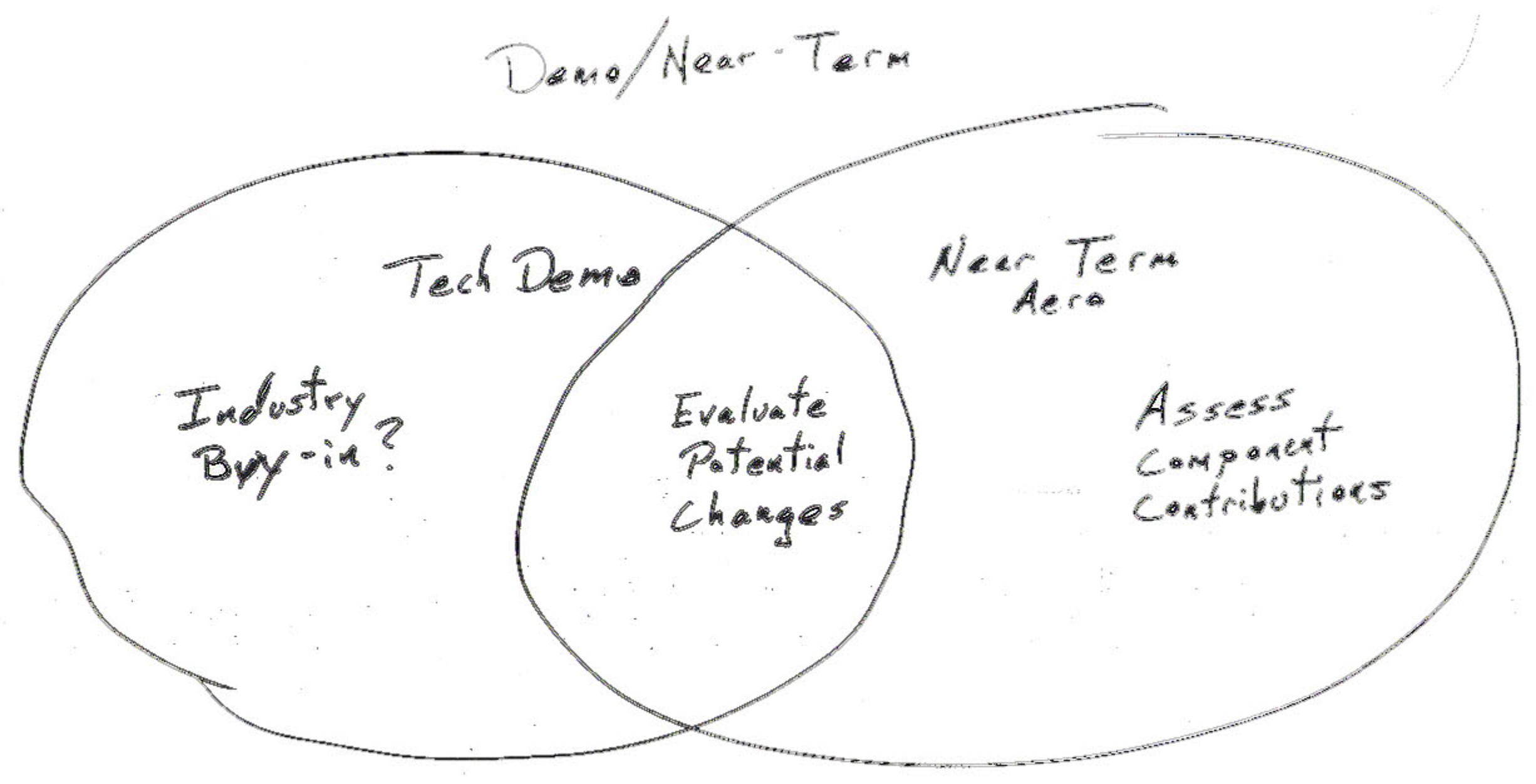


WHAT ARE the aVAILABLE CANDIDATES?

- REMOVE MIRRORS

- WIDEN CAB

- cRUSHABLE bOAT. TAIL

- CRUSHABLE NOSE cONE 
OTHER

- Wheel well tailoring

- Design guidelines

- $C_{D}$ vs. trailer skirt ground clearance
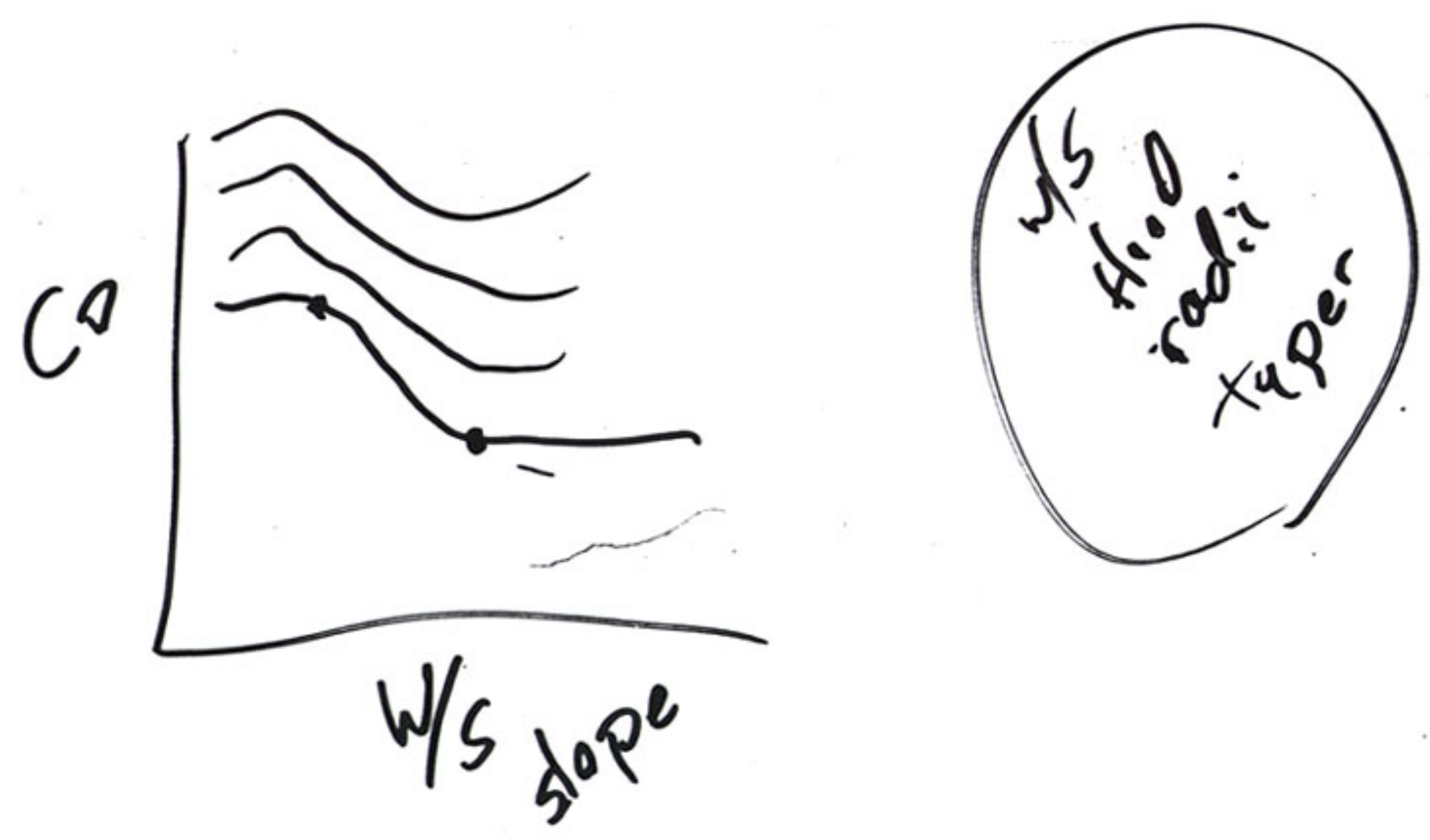